US Army Corps

of Engineers ${ }_{\circledast}$

Engineer Research and

Development Center

Engineered Resilient Systems

\title{
TradeAnalyzer v4.0 User Guide
}

Eric R. Mixon, Joshua Q. Church, Kenneth D. Niles,

July 2019 and Timothy W. Garton

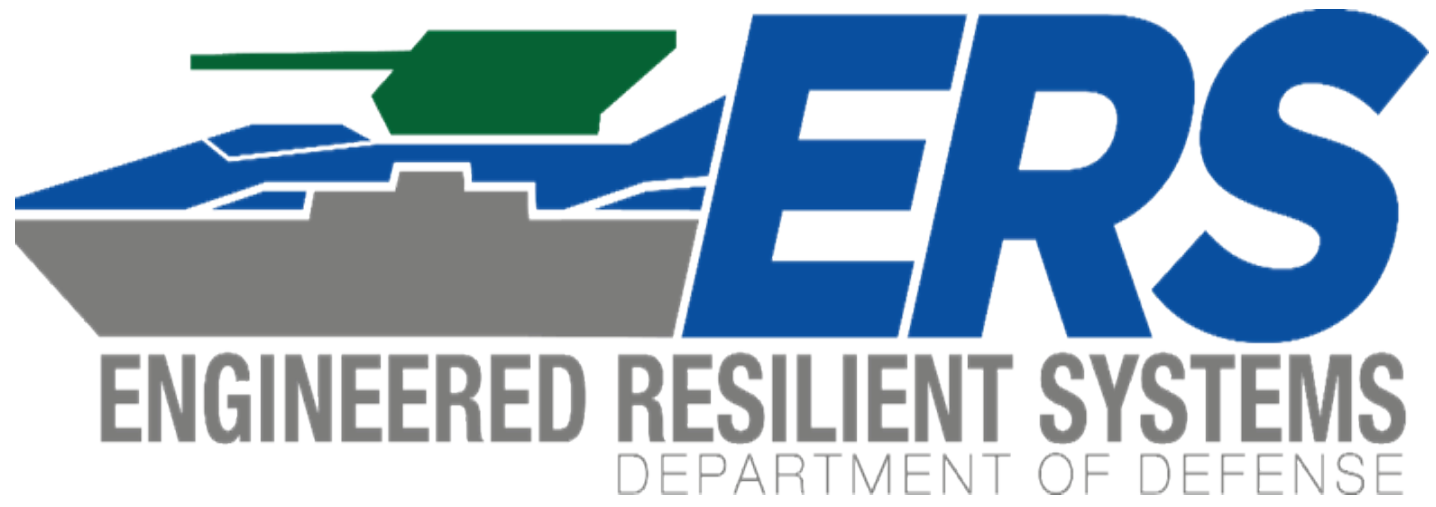


The U.S. Army Engineer Research and Development Center (ERDC) solves the nation's toughest engineering and environmental challenges. ERDC develops innovative solutions in civil and military engineering, geospatial sciences, water resources, and environmental sciences for the Army, the Department of Defense, civilian agencies, and our nation's public good. Find out more at www.erdc.usace.army.mil.

To search for other technical reports published by ERDC, visit the ERDC online library at http://acwc.sdp.sirsi.net/client/default. 


\section{TradeAnalyzer v4.0 User Guide}

Eric R. Mixon, Joshua Q. Church, Kenneth D. Niles, and Timothy W. Garton

U.S. Army Engineer Research and Development Center (ERDC) Information Technology Laboratory (ITL) 3909 Halls Ferry Road

Vicksburg, MS 39180-6199

Final Report

Approved for public release; distribution is unlimited.

Prepared for Headquarters, U.S. Army Corps of Engineers

Washington, DC 20314-1000

Under Engineered Resilient Systems Program, Data Analytics Work Package, Collaborative Tradespace Analytics Work Unit 92L5D8 


\section{Abstract}

This document describes the usage of TradeAnalyzer, a subcomponent of the overarching Engineering Resilient Systems (ERS) tool suite known as TradeStudio. TradeStudio is a generalized and reusable workflow engine used for accelerating common tradespace assembly tasks. TradeAnalyzer supports the ERS effort by developing more comprehensive and complete tradespaces, facilitating Department of Defense (DoD) conceptual design pipelines to the High Performance Computer (HPC), and by solving cross cutting issues in the design process.

TradeAnalyzer serves as an enterprise level web application designed to assist the user in visualizing and analyzing a tradespace. The analytics and visualizations provided act as a suite for decision support in down selecting to the best possible subset for the mission. The products provided by TradeAnalyzer include Large Data Analytics and Visualization (LDV) tools which use high performance, server-side rendering.

DISCLAIMER: The contents of this report are not to be used for advertising, publication, or promotional purposes. Citation of trade names does not constitute an official endorsement or approval of the use of such commercial products. All product names and trademarks cited are the property of their respective owners. The findings of this report are not to be construed as an official Department of the Army position unless so designated by other authorized documents. 


\section{Contents}

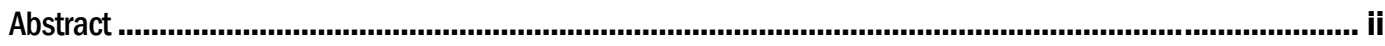

Figures and Tables............................................................................................................................................

Preface....................................................................................................................................................vili

Acronyms and Abbreviations..............................................................................................................ix

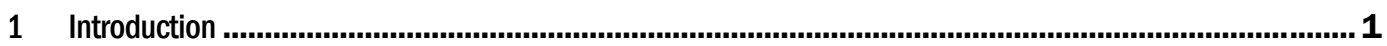

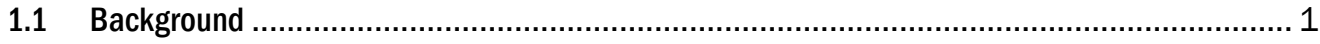

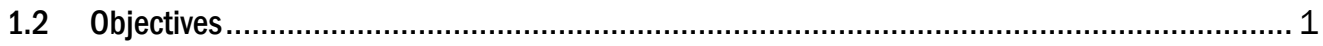

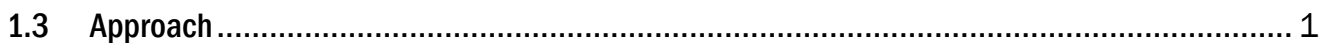

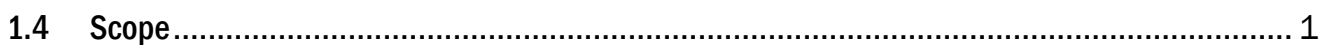

2 Account Creation ................................................................................................................... 2

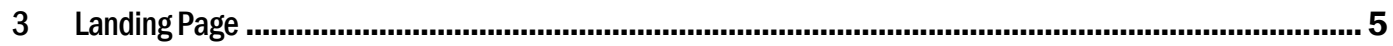

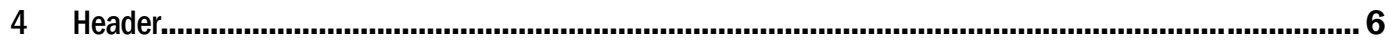

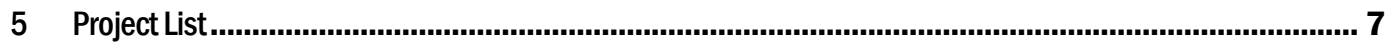

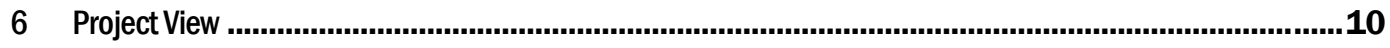

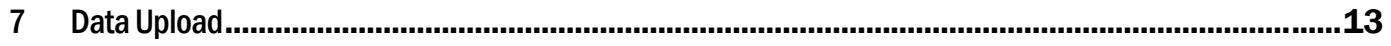

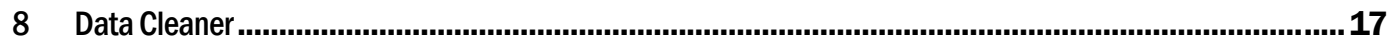

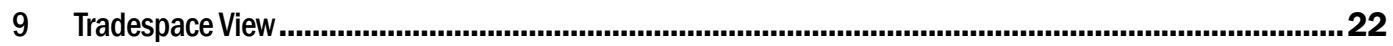

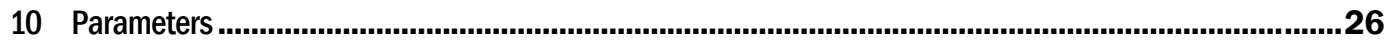

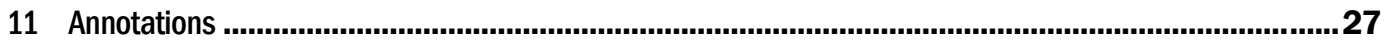

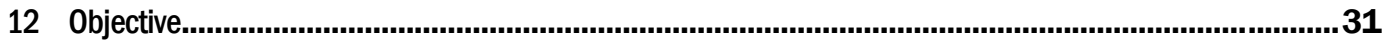

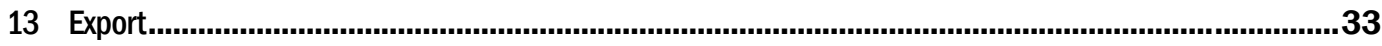

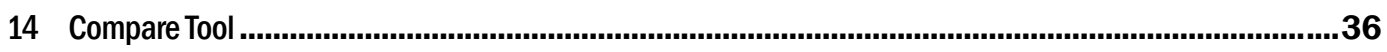

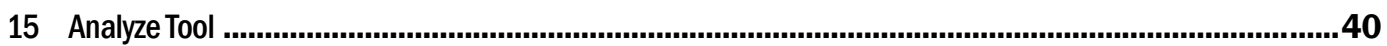

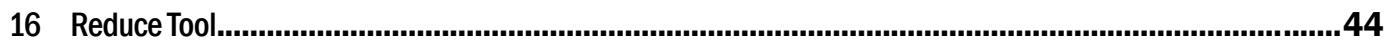

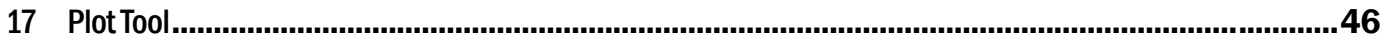

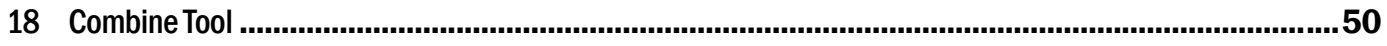

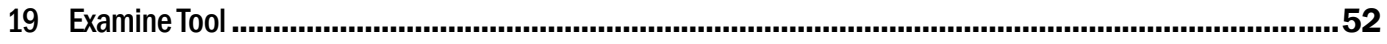




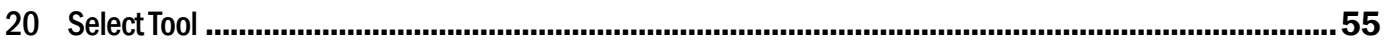

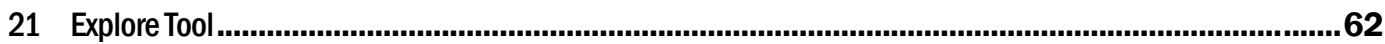

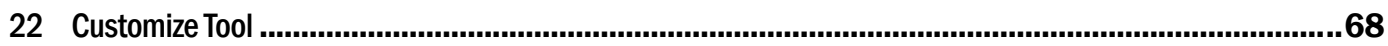

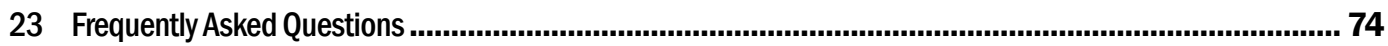

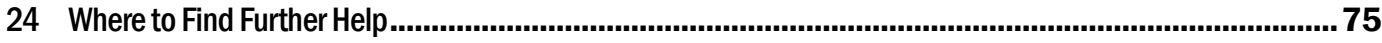

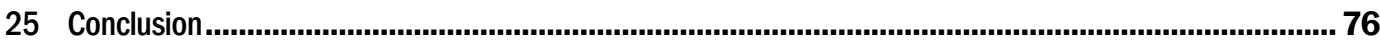

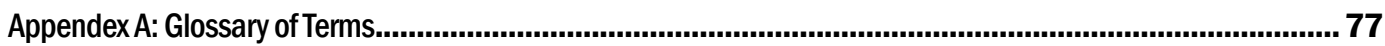

Report Documentation Page 


\section{Figures and Tables}

\section{Figures}

Figure 1. Selecting an authentication method. ................................................................................. 3

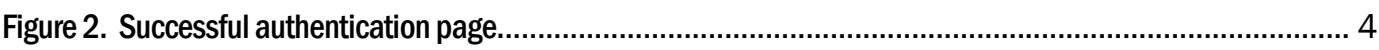

Figure 3. TradeStudio landing page. ............................................................................................ 5

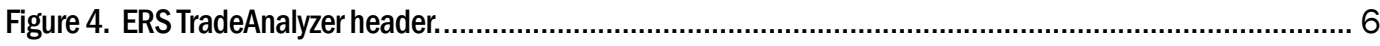

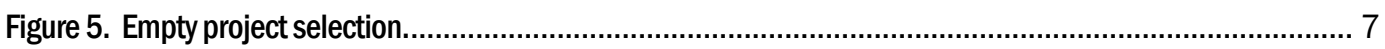

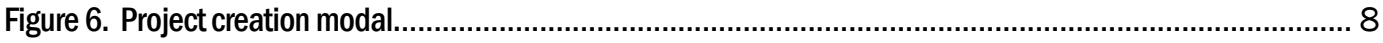

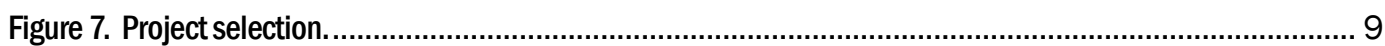

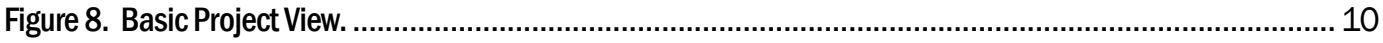

Figure 9. Project permissions................................................................................................... 11

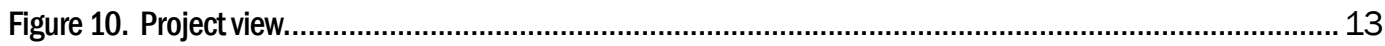

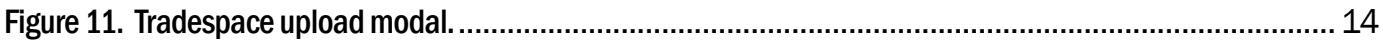

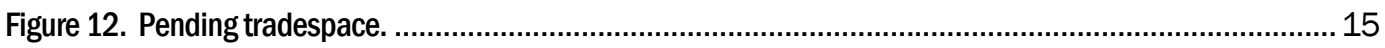

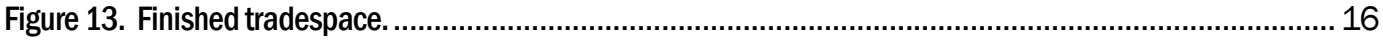

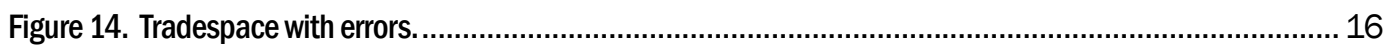

Figure 15. Tradespace with errors........................................................................................... 17

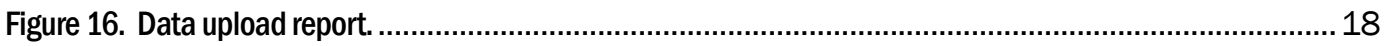

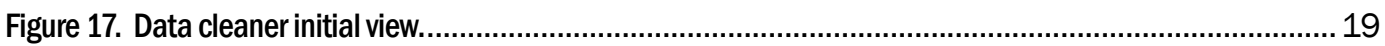

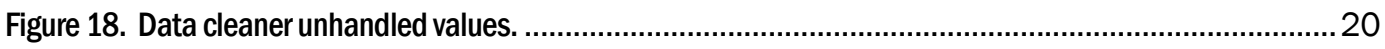

Figure 19. Data cleaner editing values. ........................................................................................... 21

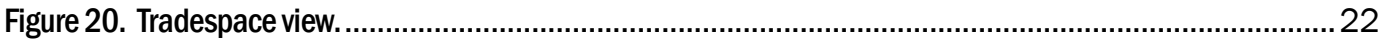

Figure 21. Tradespace view with subset.......................................................................................... 23

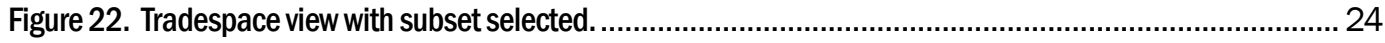

Figure 23. Tradespace view with snapshot...................................................................................... 24

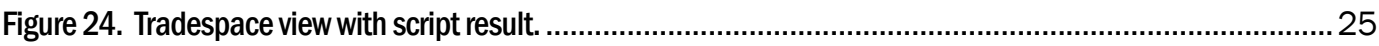

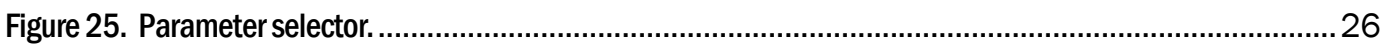

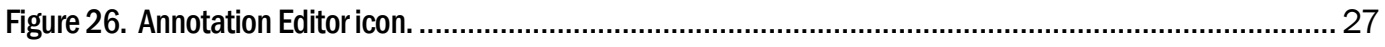

Figure 27. Adding a divider..................................................................................................... 28

Figure 28. Annotation Editor - partition....................................................................................... 29

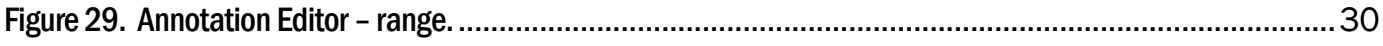

Figure 30. Objective Function Icon................................................................................................. 31

Figure 31. Objective Function Builder........................................................................................ 31

Figure 32. Export Button Location .............................................................................................. 33

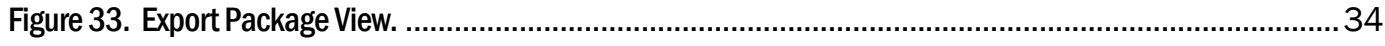

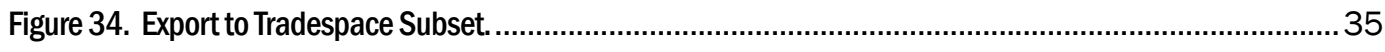

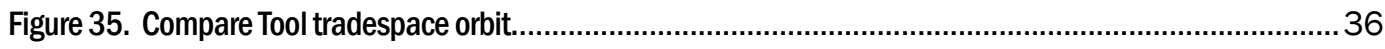

Figure 36. Compare Tool selection view. .............................................................................................. 37 


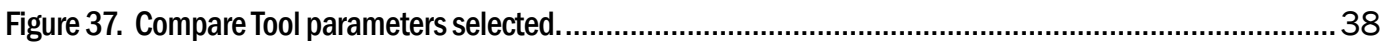

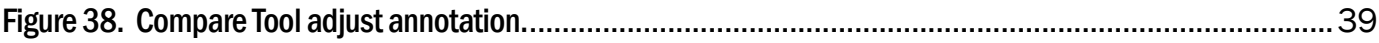

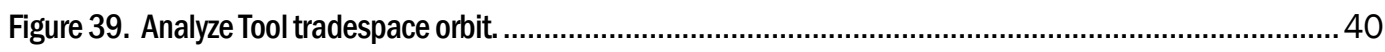

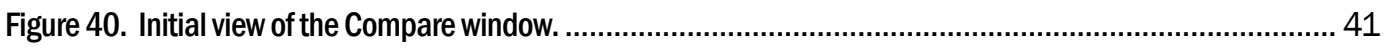

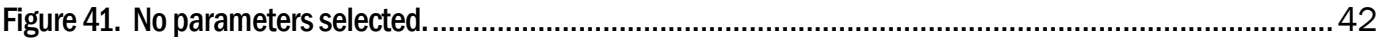

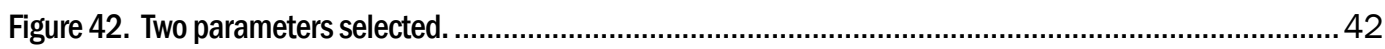

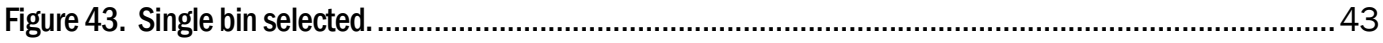

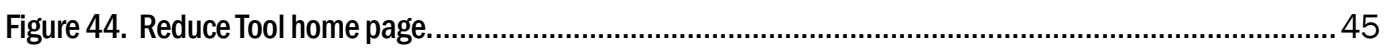

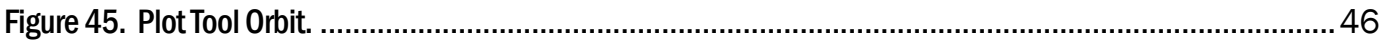

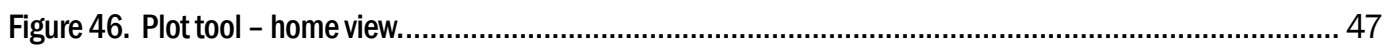

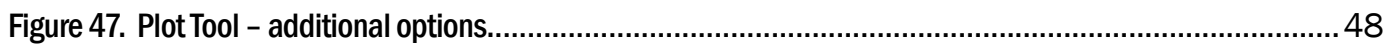

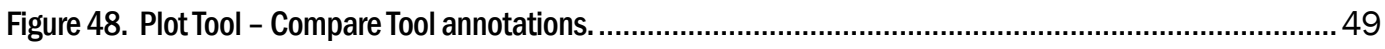

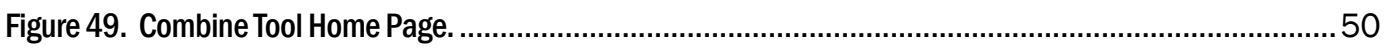

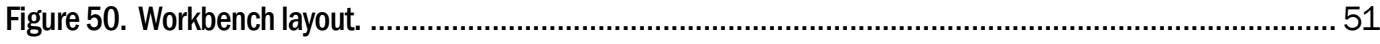

Figure 51. Examine tool no parameters selected. ....................................................................................52

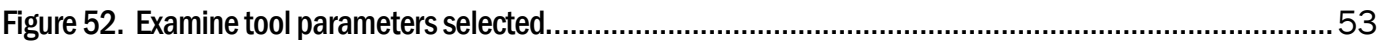

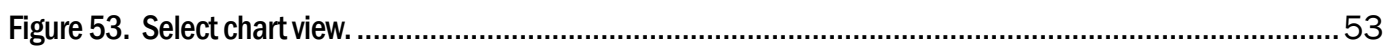

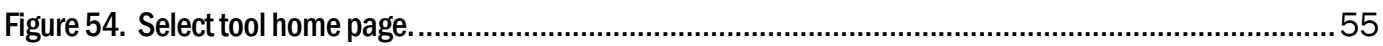

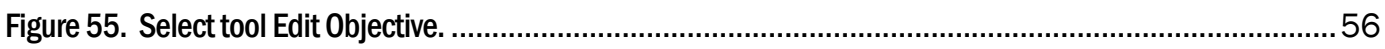

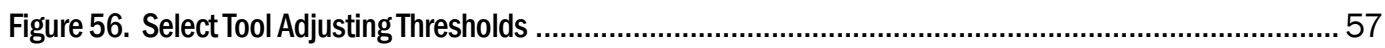

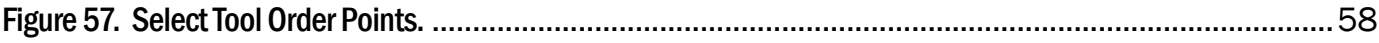

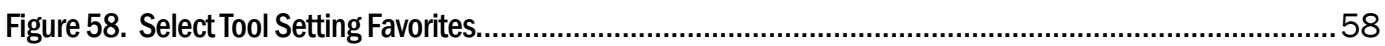

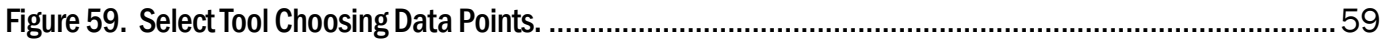

Figure 60. Select Tool Points Comparison Page ......................................................................................6 60

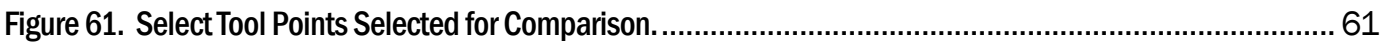

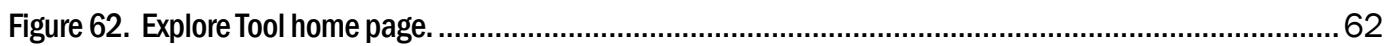

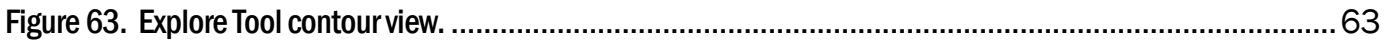

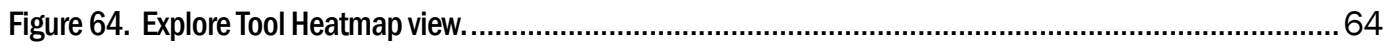

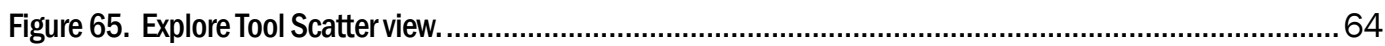

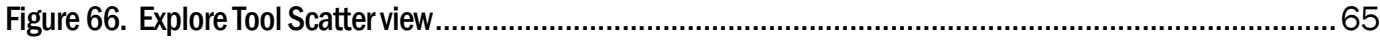

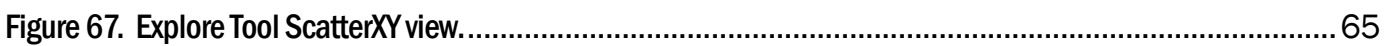

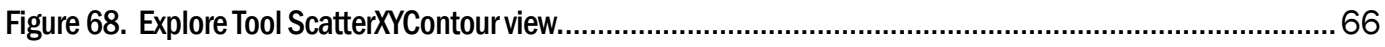

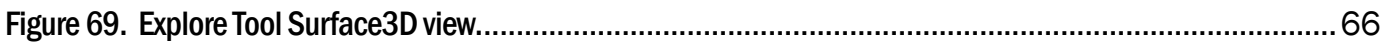

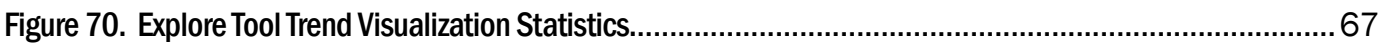

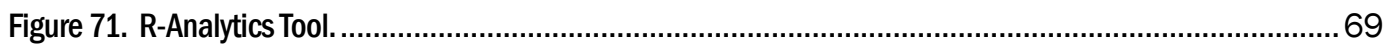

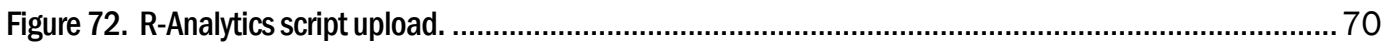

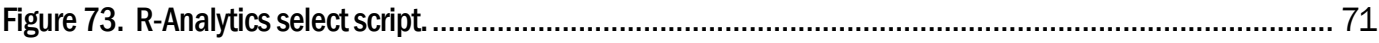

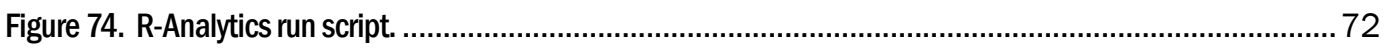

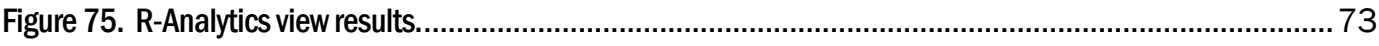

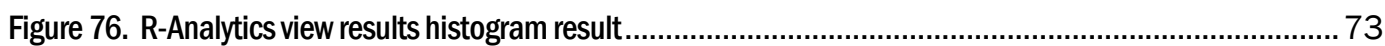




\section{Tables}

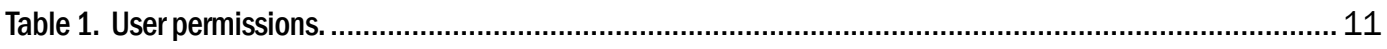




\section{Preface}

This users guide is a deliverable product under the Engineered Resilient Systems (ERS) Program, Data Analytics Work Package, Collaborative Tradespace Analytics Work Unit 92L5D8. Dr. Owen J. Eslinger was the program manager, and Dr. Robert M. Wallace was the Technical Director of the ERS program.

The work was performed by the Scientific Software Branch (SSB) and Computational Analysis Branch (CAB) of the Computational Science and Engineering Division (CSED), Engineer Research and Development Center (ERDC), Information Technology Laboratory (ITL), Vicksburg, MS. At the time of publication, Mr. Timothy Dunaway was Chief, SSB, and Dr. Jeffrey L. Hensley was Chief, CAB. Dr. Jerrell R. Ballard was the Chief, CSED. The Deputy Director of ITL was Ms. Patti S. Duett and the Director was Dr. David A. Horner.

COL Ivan P. Beckman was the Commander of ERDC, and Dr. David W. Pittman was the Director. 


\section{Acronyms and Abbreviations}

\begin{tabular}{|l|l|}
\hline Term & Meaning \\
\hline 2D & Two-Dimensional \\
\hline 3D & Three-Dimensional \\
\hline CAB & Computational Analysis Branch \\
\hline CAC & Common Access Card \\
\hline CSED & Computational Science and Engineering Division \\
\hline CSV & Comma Separated Values \\
\hline DoD & Department of Defense \\
\hline EDIPI & Electronic Data Interchange Personal Identifier \\
\hline ERDC & Engineer Research and Development Center \\
\hline ERS & Engineered Resilient Systems \\
\hline HPC & High Performance Computing \\
\hline ITL & Information Technology Laboratory \\
\hline LDV & Lightweight Database Virtualization \\
\hline PIN & Personal Identification Number \\
\hline PING & PingFederate \\
\hline POC & Point of Contact \\
\hline RDE & Research and Development Environment \\
\hline SSB & Scientific Software Branch \\
\hline USACE & U.S. Army Corps of Engineers \\
\hline
\end{tabular}




\section{Introduction}

\subsection{Background}

This report serves as a user guide to understanding the various views and tools in TradeAnalyzer. The guide provides thorough explanations and example use cases of the many tools TradeAnalyzer provides. It is recommended that the user begin by visiting the website and follow the built-in tutorials. The user should reference this guide whenever there is need for more information or case examples.

A few notes to the reader:

- The color scheme of TradeAnalyzer does not include red, the red boxes and text on screenshots of this document are for illustration only.

- The word "Parameter" refers to a selected portion of data from uploaded tradespaces. Some parts of the guide and web application might also refer to this as "Column" or "Field." All of these simply represent a particular selection of data.

\subsection{Objectives}

This report is a user guide intended to aid new users through the workflow and usage of TradeAnalyzer.

\subsection{Approach}

This document guides the user through account creation and works progressively through the TradeAnalyzer tools in the order that they would be encountered. In this way, readers will have a sense of the expected work flow of the tool and how it is anticipated to be used.

\subsection{Scope}

The scope of this document will focus strictly on usage of TradeAnalyzer from being a new user to being an expert in all aspects of the tool. 


\section{Account Creation}

Creating a PingFederate (Ping) account is the first step to getting access to TradeAnalyzer. The user or the user's Engineer Research and Development Center (ERDC) Point of Contact (POC) may request a new Ping account for the Engineered Resilient Systems (ERS) TradeAnalyzer by sending an email to ERS.Support@erdc.dren.mil. All account requests must include the following information:

- Name

- E-mail

- Organization

- ERDC POC

- Electronic Data Interchange Personal Identifier (EDIPI) or Statement of Non-Common Access Card (CAC) status

- Status of Information Assurance (IA) training if not a CAC holder.

- Information about the user's organization's network.

Additionally, after the initial request is made, the user will be provided with form PING DD-2875 to be completed and returned to the Research and Development Environment (RDE) HelpDesk (RDEHelpDesk@erdc.dren.mil). A ticket number will be issued and used to track the progress of the account application. Once accepted, the request will provide a Ping account limited to TradeAnalyzer and other TradeStudio tools.

All CAC holders will receive CAC-required accounts. These accounts do not expire and do not have a password associated with them. The account will remain active as long as the $\mathrm{CAC}$ remains active. The $\mathrm{CAC}$ will be used for authentication and authorization. If certain network policies prevent CAC-based authentication, non CAC authentication can be used.

Non CAC authentication can be used if the user does not possess an active CAC or external forces prevent the use of CAC as an authentication method. Non CAC two-factor authentication uses a username and password combined with a cellphone text message to identify the user. The username and password will expire after sixty days, and may only be reset via the RDE HelpDesk. Notice of this expiration will be e-mailed to the address provided during account creation. If the password is not reset before expiration, the account will not be accessible, even if a valid CAC is attached to the account. 
Once an account is created, a user can test their access by going to https://tradestudio.ers.erdc.dren.mil/connection. There will be a page asking the user to select their authentication method (Figure 1).

Figure 1. Selecting an authentication method.

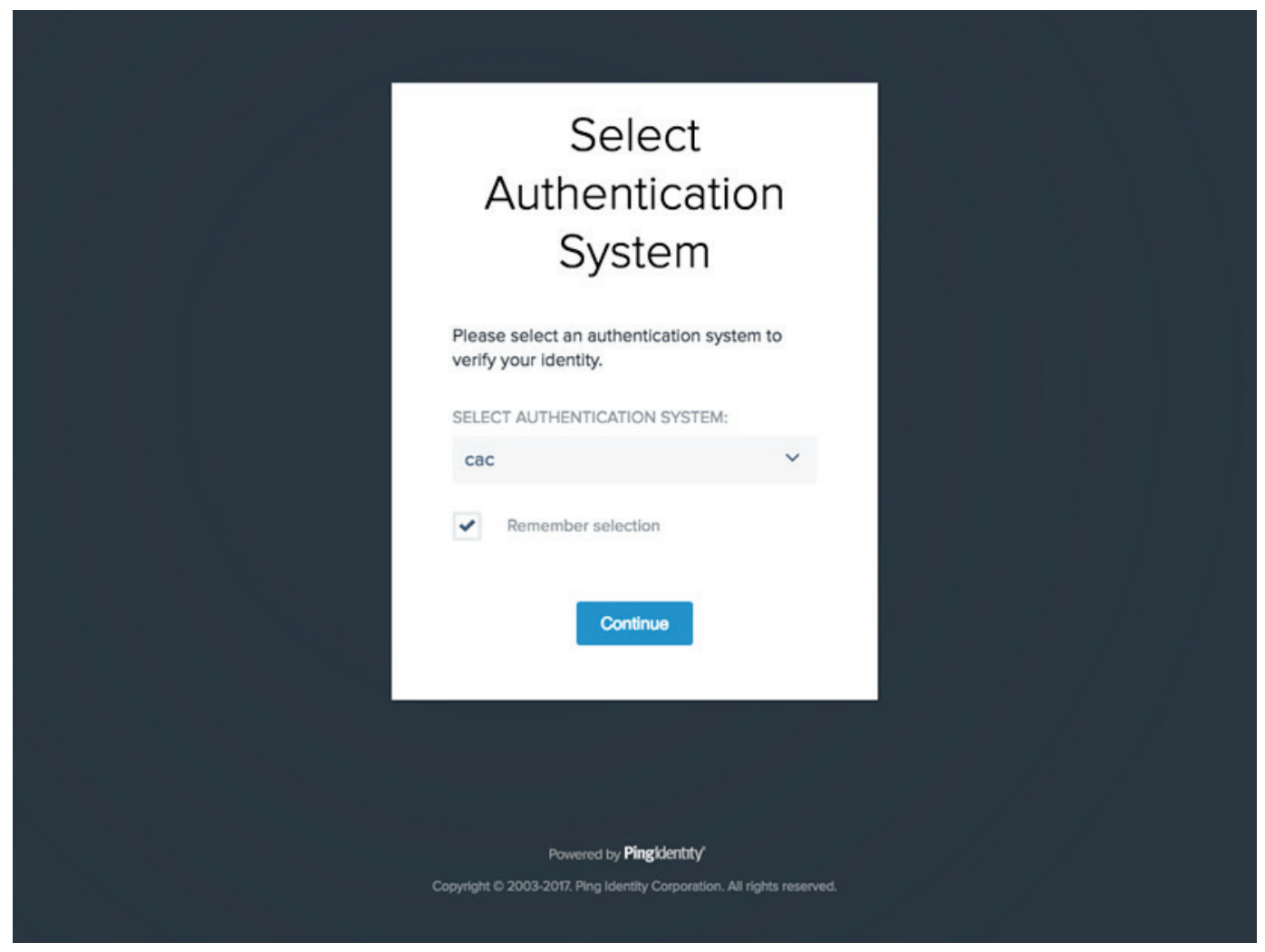

If the user has an active account with a CAC associated with it, they can log in by selecting the cac option. To continue, the user should select their CAC certificate and enter their CAC Personal Identification Number (PIN). If the user does not have a CAC associated with their account, they should provide their username, password, and the provided code texted to their phone.

After a successful login, the user will see the successful authentication page (Figure 2) confirming their access to TradeStudio. 
Figure 2. Successful authentication page.

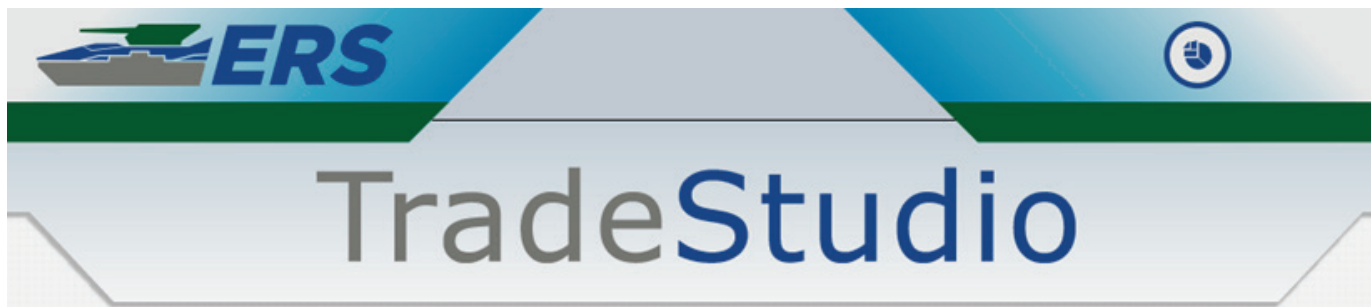

A software suite used to define your design space, set element parameters, optimize the output for tradespace creation, and analyze, narrow, and select designs

\section{You have successfully connected to ERS TradeStudio.}




\section{Landing Page}

When a user first logs in, they are presented with the TradeStudio landing page (Figure 3). This page provides generic information about the tools in TradeStudio. Currently, the landing page provides information about both the TradeBuilder and TradeAnalyzer tools. For this guide, the focus will be on TradeAnalyzer. To navigate to the TradeAnalyzer tool, simply click the button under the TradeAnalyzer section labeled OPEN (Figure 3).

Figure 3. TradeStudio landing page.

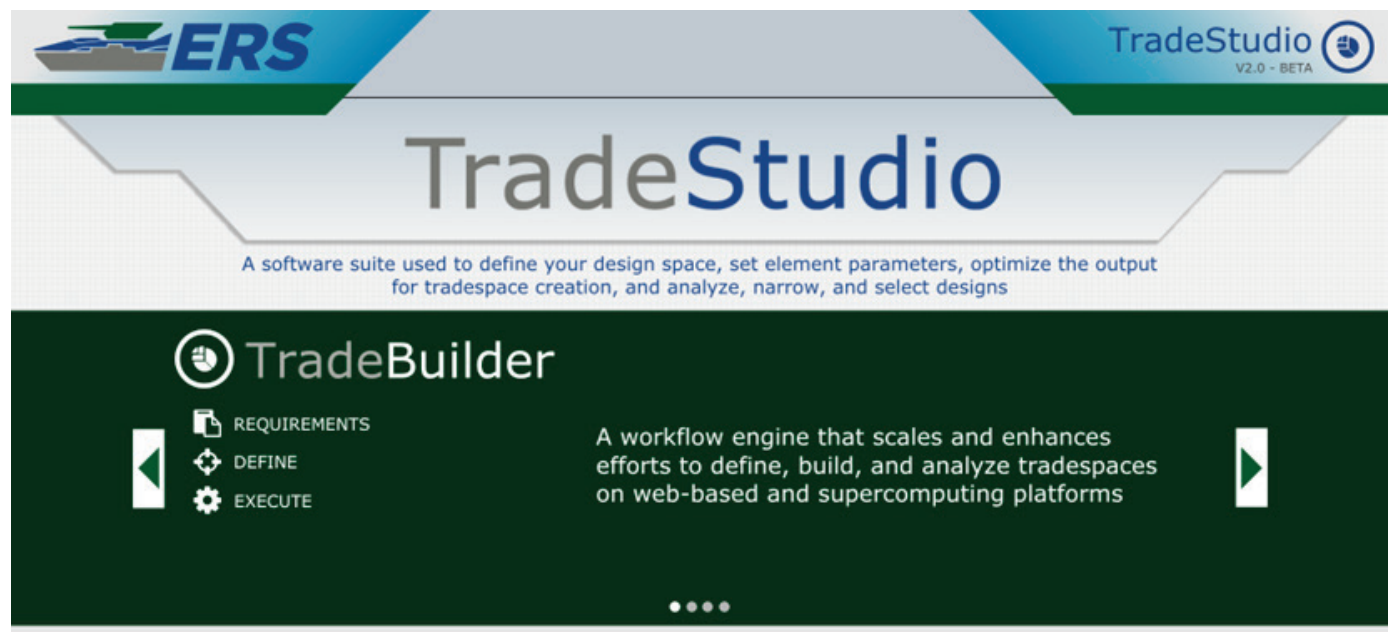

\section{(:) TradeAnalyzer}

An enterprise-level web portal designed

to assist you in visualizing and

() Visualize

analyzing a tradespace

$$
\text { Decision Support }
$$




\section{Header}

After navigating to TradeAnalyzer, the user is presented with the Project List. At the top of the page is the header, this provides common functionality found on every view of the site (Figure 4).

Figure 4. ERS TradeAnalyzer header.

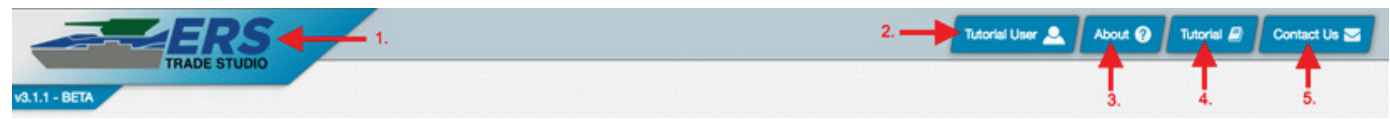

The header contains the following parts:

1. ERS Logo - The ERS TradeStudio logo in the top left portion of the screen can be clicked to return to the Project List. Note: this will deselect any loaded projects or tradespaces and remove any unsaved work. If a tradespace is loaded, the name will appear to the right of the logo.

2. User Name - The user's name will appear here. If the user has unread notifications, they will appear as a number next to the user icon.

3. About Page - The About button will show a modal providing a general overview of TradeAnalyzer.

4. Tutorials - Many pages have interactive tutorials. When available, the Tutorial button will take the user through a guided tour of the page, showing what interactivity is available. Users are recommended to use tutorials when available if they are having trouble on a specific page.

5. Contact Us - The Contact Us button lets the user contact the TradeAnalyzer team with comments, concerns, or complements. 


\section{Project List}

When the user first opens the TradeAnalyzer tool, they see the project list (Figure 5). A project is a collection of all the components a user needs to analyze and visualize created tradespaces. The project includes tradespace data, subsets of tradespaces, permission for team members, snapshots of objective states, custom scripts, and analytical results.

Figure 5. Empty project selection.

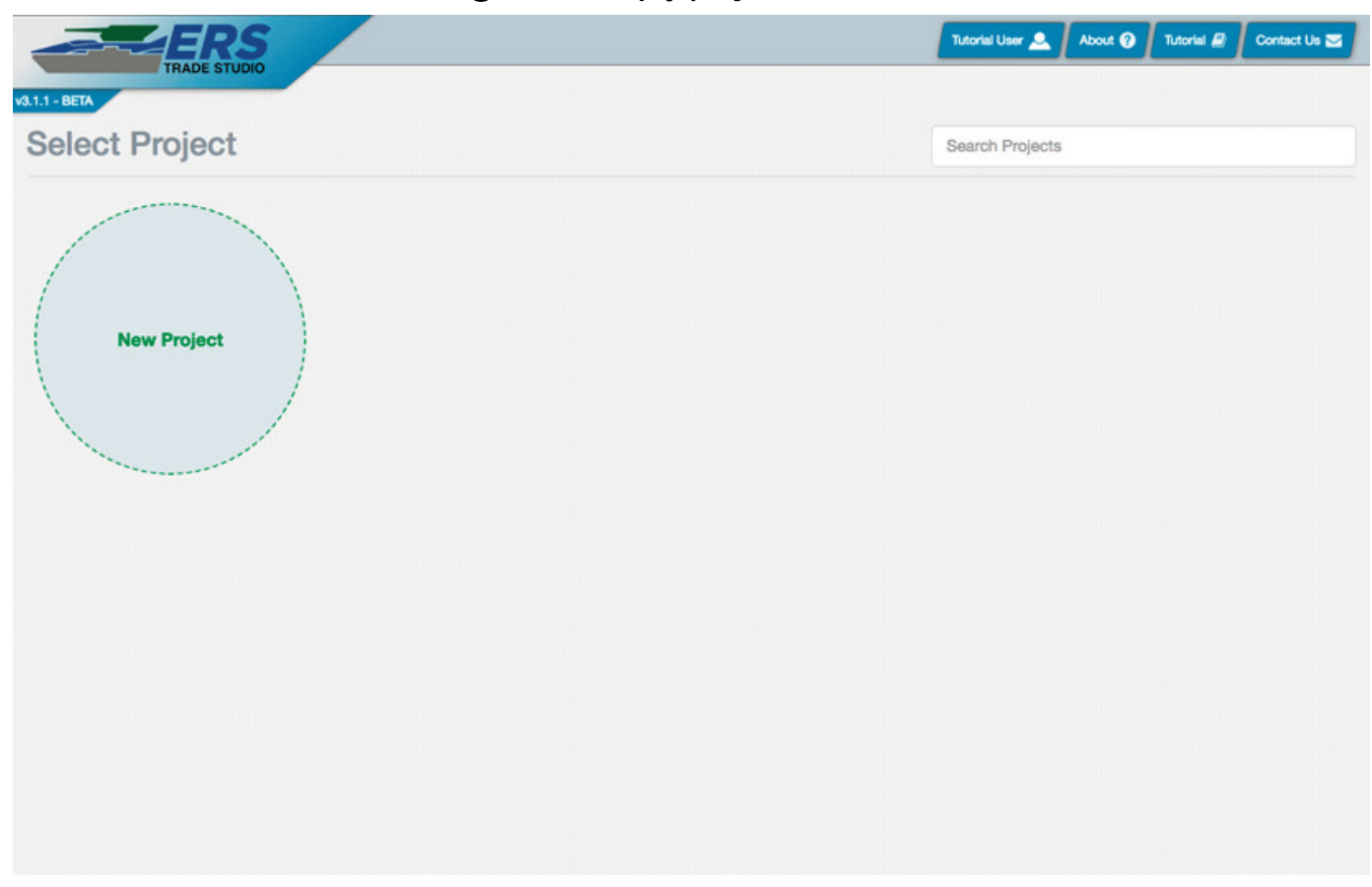

When first visiting TradeAnalyzer, a user does not have access to any projects. In order for a new user to be given access to a project, they will need to visit the page at least once. For this guide, the user will be creating a new project.

Clicking the New Project icon presents the user with the project creation modal (Figure 6). 
Figure 6. Project creation modal.

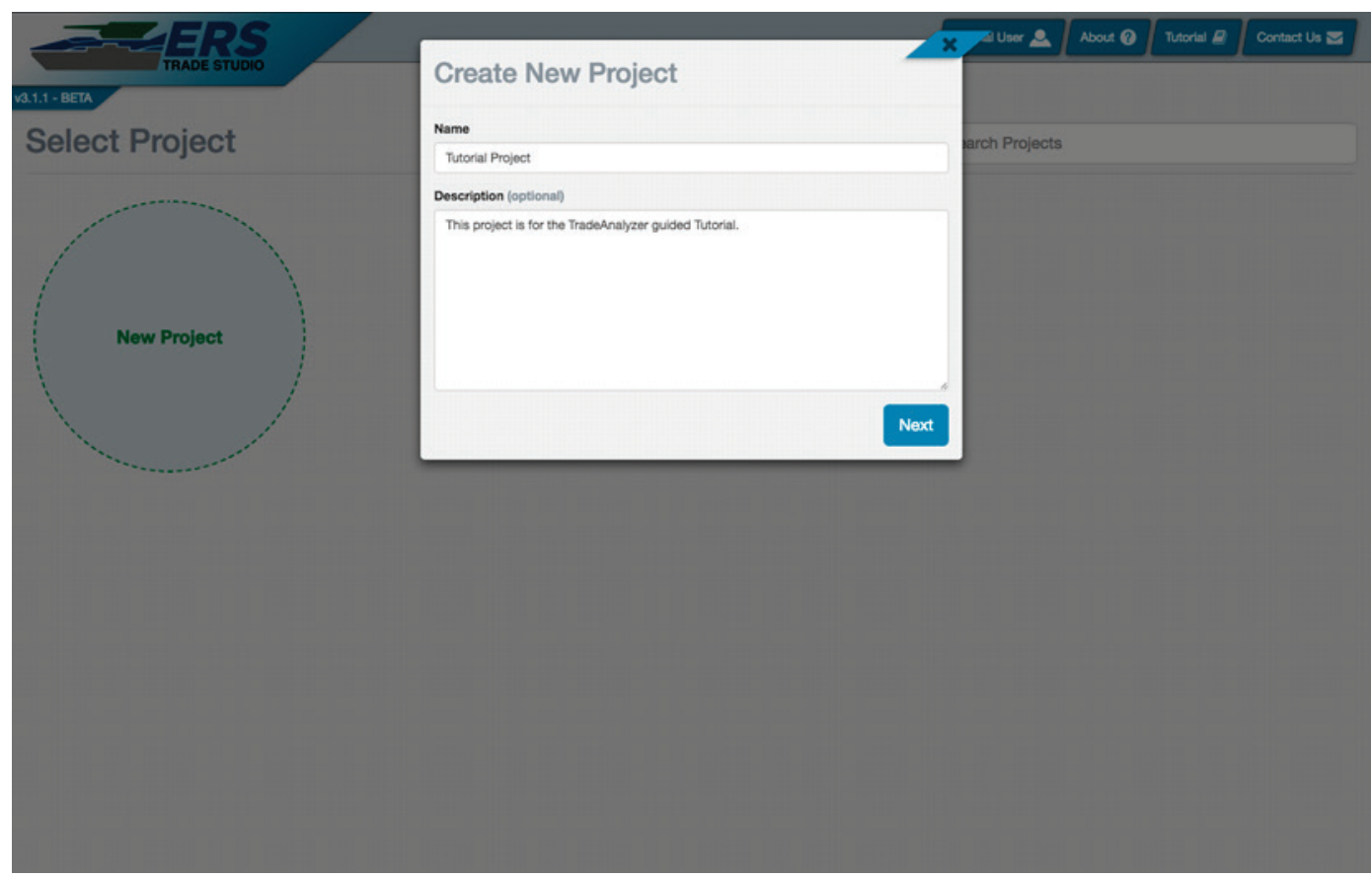

The project creation modal asks for a name and an optional description. The user should name their project with a short name that identifies the project's purpose. Any additional details can be added to the description, if desired. Once the new project has been named, choosing Next finishes creating the project.

Once the user chooses Next, this automatically takes them to the Project View for their newly created project. To return to project selection, the user can either use the ERS TradeStudio Logo in the top left portion of the screen or the semicircle on the left side of the screen in the Project View.

The project selection will now display the newly created project (Figure 7). To open the project from this view, the user clicks the circle labeled with the name of the newly created project. For convenience, projects are assigned a consistent color to make it easier to identify the project. 
Figure 7. Project selection.

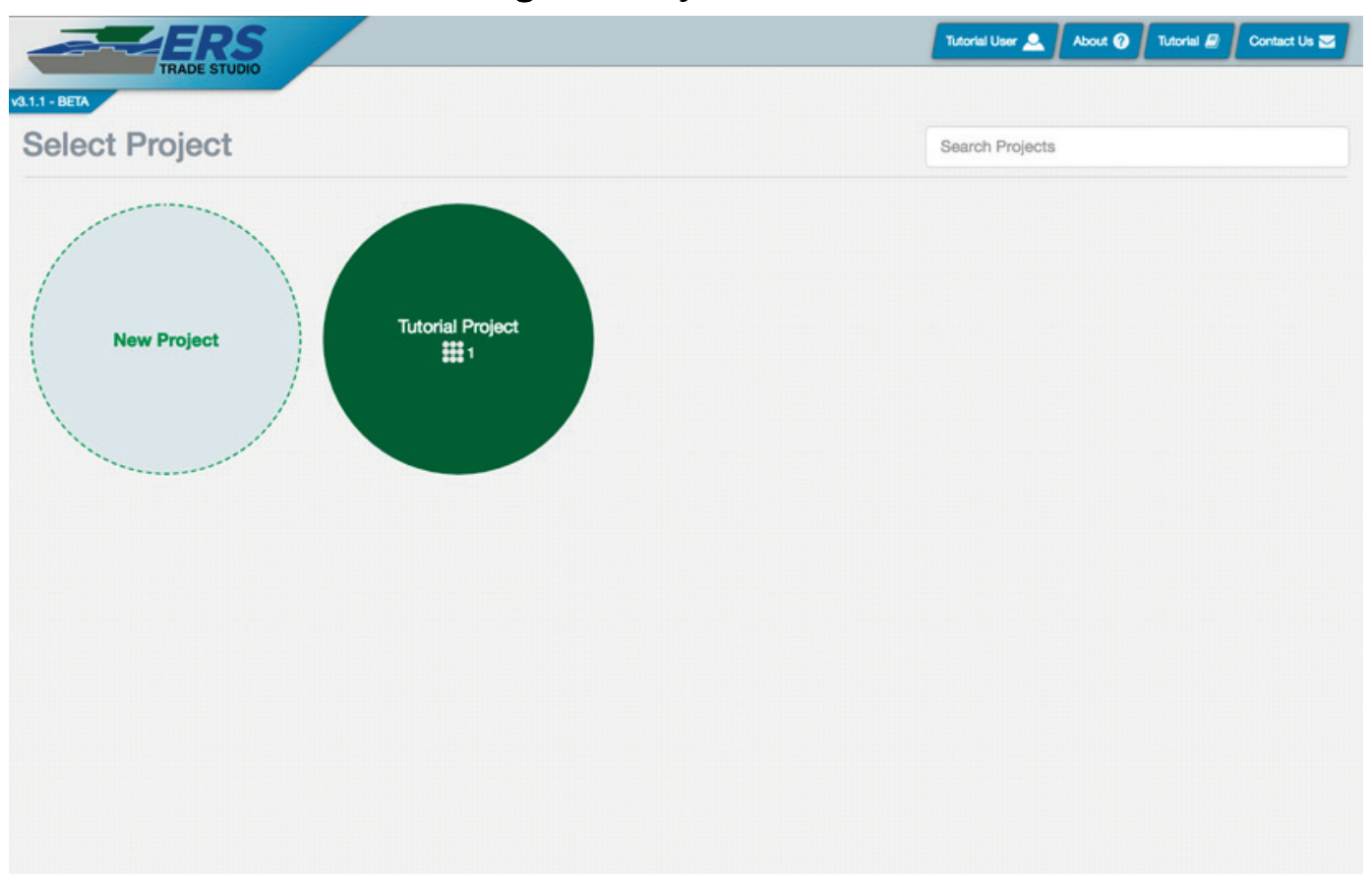

The number displayed next to the grid icon on the project circle represents the number of tradespaces that exist in this project. In Figure 7, the number shows one, this indicates a project contains one tradespace. A newly created project will have zero tradespaces.

Also worth noting is the "Search Projects" tool to the right of Select Project. If a user has many projects, typing the name of the project in the Search Projects box will narrow the number of projects on the screen down to only projects that have the typed name.

To proceed to the Project View, the user should click on their desired project. This will load the project. 


\section{Project View}

The Project View (Figure 8) contains all of the current tradespaces within that project and tools to edit the project. A user can change the name or description, upload new tradespaces, and allow other users to access the project.

Figure 8. Basic Project View.

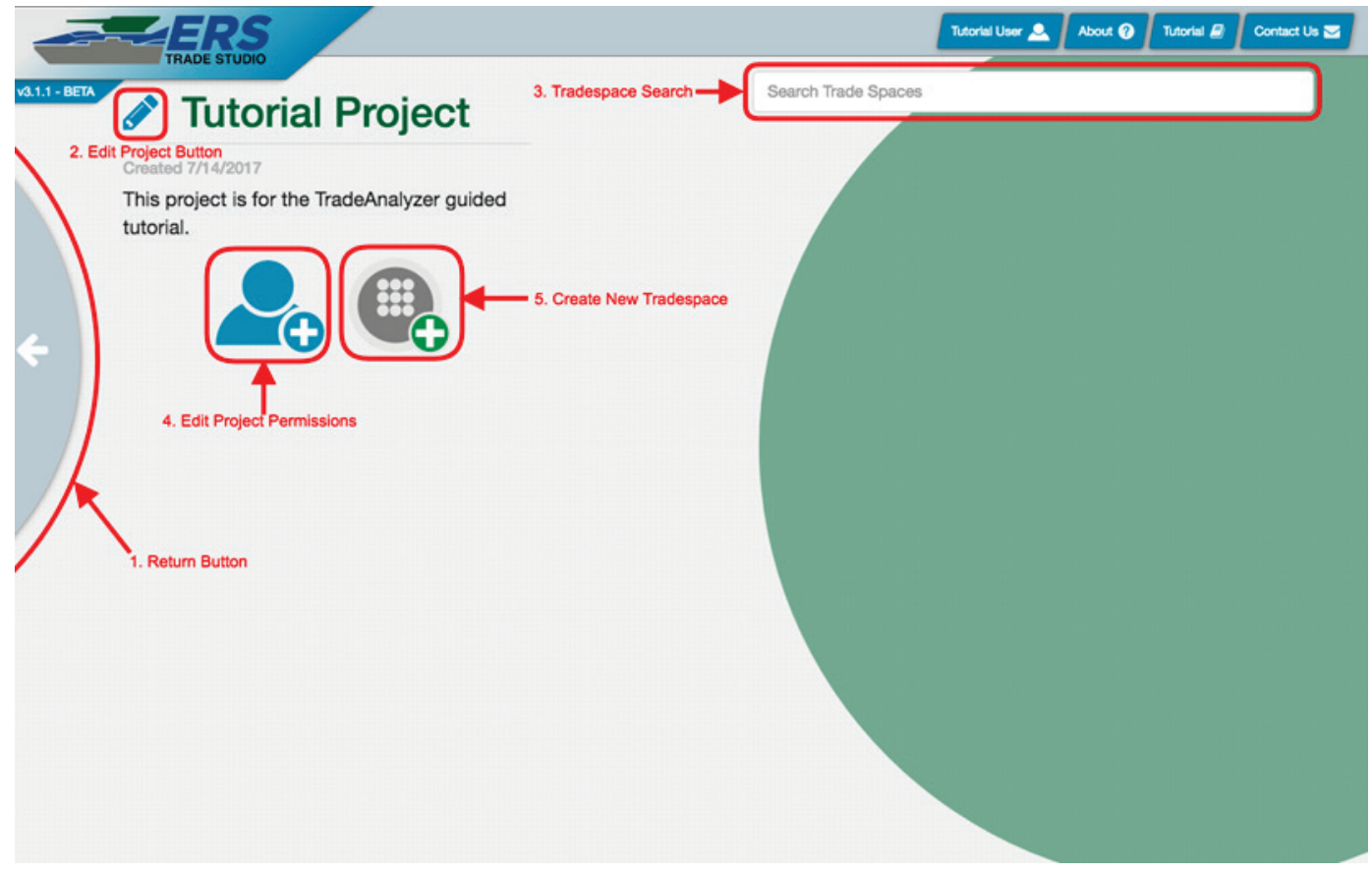

Selecting the pencil icon next to the project name allows the user to change the project name and description. This icon is hidden to users with the role of "Guest" as shown in Table 1.

If there are many tradespaces, typing the name of the tradespace in the Search Trade Spaces box narrows the number of tradespaces on the screen down to only tradespaces that have the typed name.

Below the project name and project description are the user permissions and the add tradespace icons. Clicking the user permissions icon opens the user permissions modal (Figure 9). The add tradespace icon will be discussed in the Data Upload section. 
Figure 9. Project permissions.

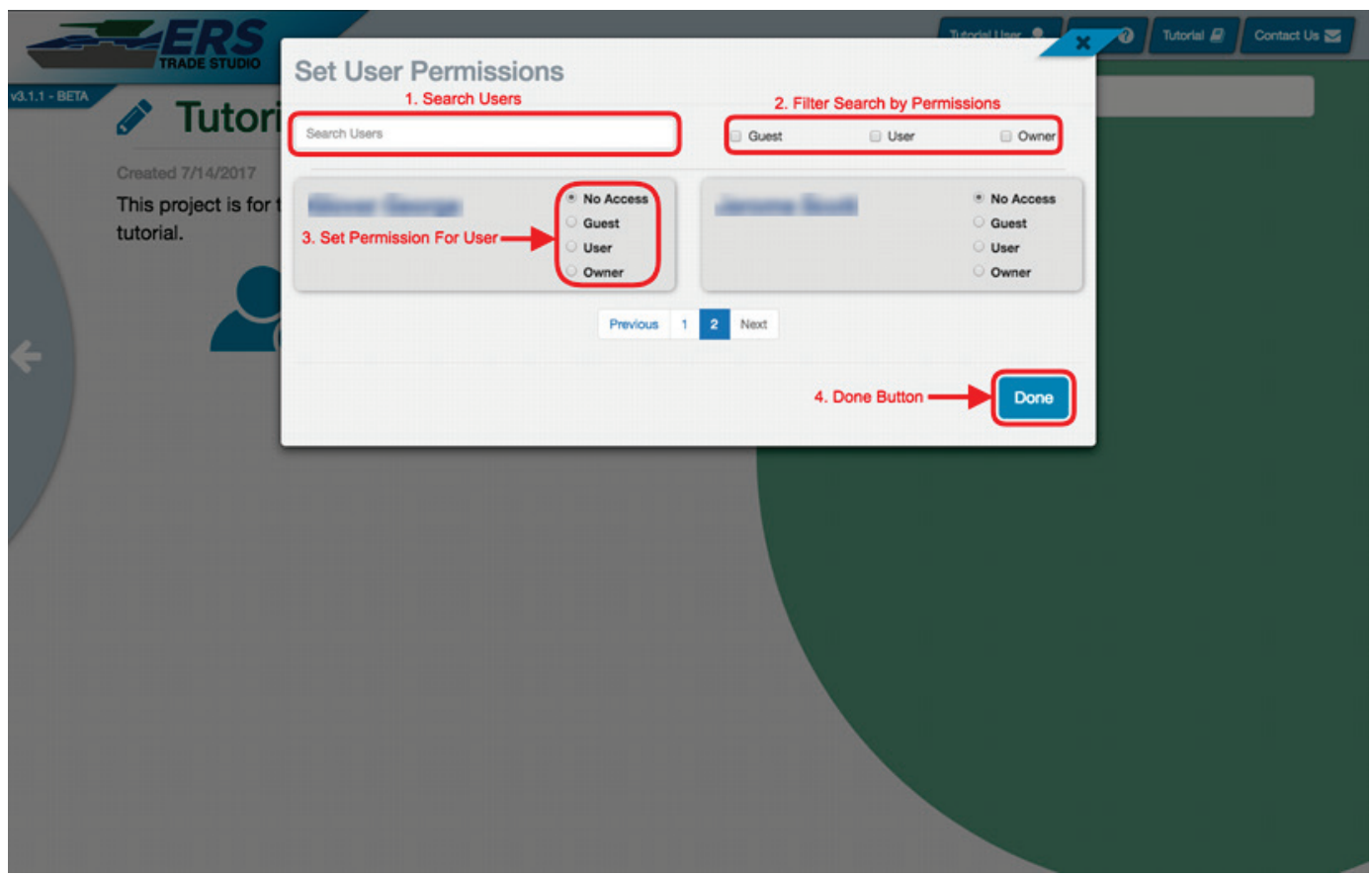

The user permissions modal has a list of all users who have previously logged into the TradeAnalyzer tool and allows owners of projects to grant user permissions to these users. If a user does not have "owner" permission on the project, they will not see the user permissions icon.

Search Users will allow the owner to search for any user they want. To the right of Search Users are filters that allow an owner to show only guests, users, or owners. By default, all users in the system have no access to an owners' project. However, there are a number of permission settings that can be granted (Table 1).

Table 1. User permissions.

\begin{tabular}{|l|l|}
\hline \multicolumn{1}{|c|}{ User Permission } & \multicolumn{1}{c|}{ Rights } \\
\hline Guest & View Only \\
\hline User & View / Modify \\
& Cannot Edit User Permissions \\
\hline Owner & View / Modify / Grant \\
\hline
\end{tabular}

Guest permissions allow a user to view and interact with a project, but the user cannot save or modify any component of the project. User permissions allow a user to view, interact, save, and modify the project, but they will have no ability to modify permissions for users. Owner 
permissions allow a user the same permissions as the project owner, including the ability to modify permissions for other users. At any time, a user can hover over No Access, Guest, User, or Owner to view information about these roles and what permissions they grant. 


\section{Data Upload}

In order to use many of the tools in TradeAnalyzer, a user needs data to analyze. The main way to access data is by uploading a Comma Separated Values (CSV) file to a project. Once a user has created a project, they upload a dataset using the tradespace upload icon in the Project View (Figure 10).

Figure 10. Project view.

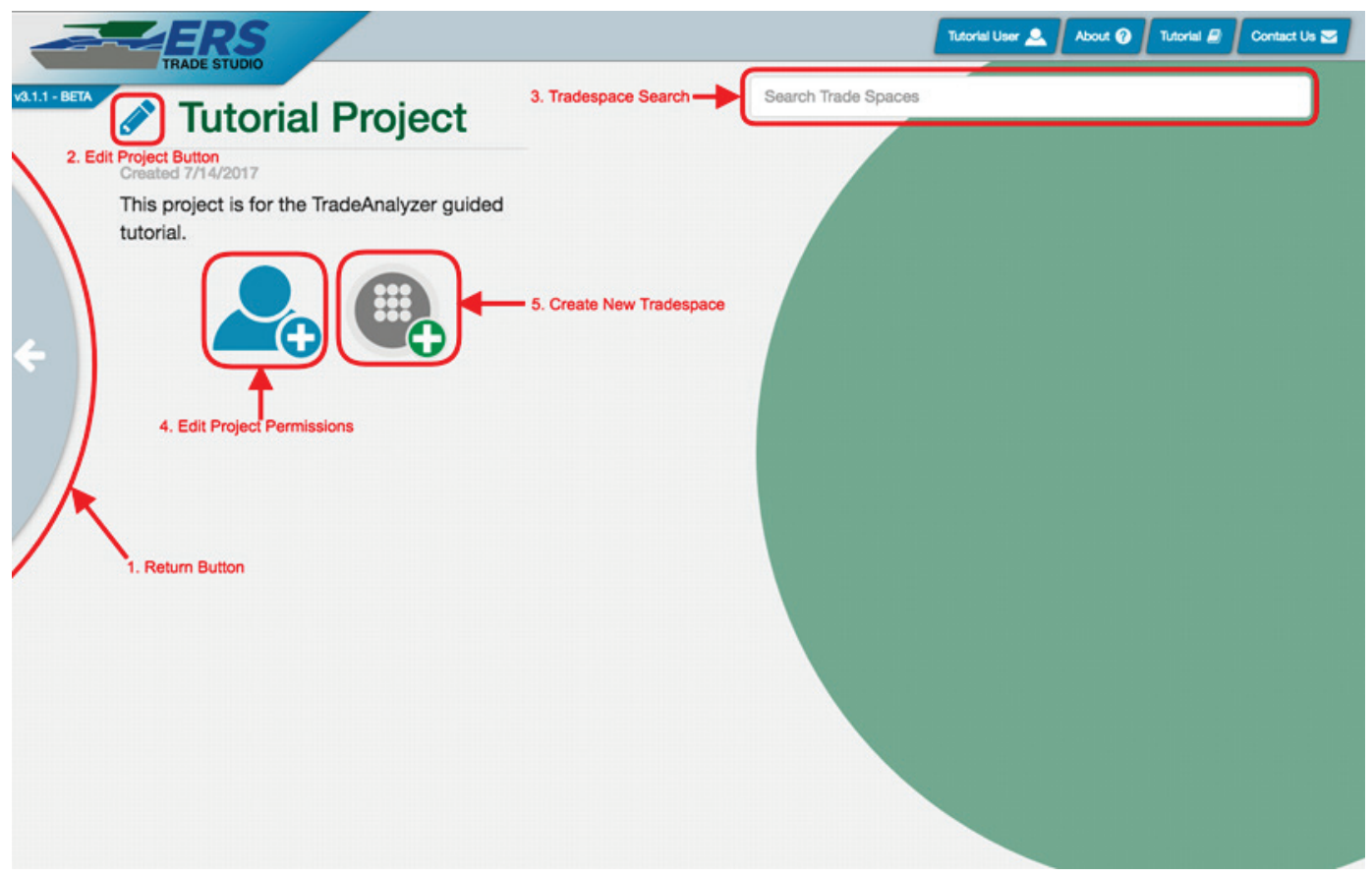

Clicking on this icon displays the Tradespace Upload Modal (Figure 11). This modal allows user to select a CSV file for upload and provides a name and optional description for your new tradespace. The name of the tradespace should be short, but represent the content of the data. 
Figure 11. Tradespace upload modal.

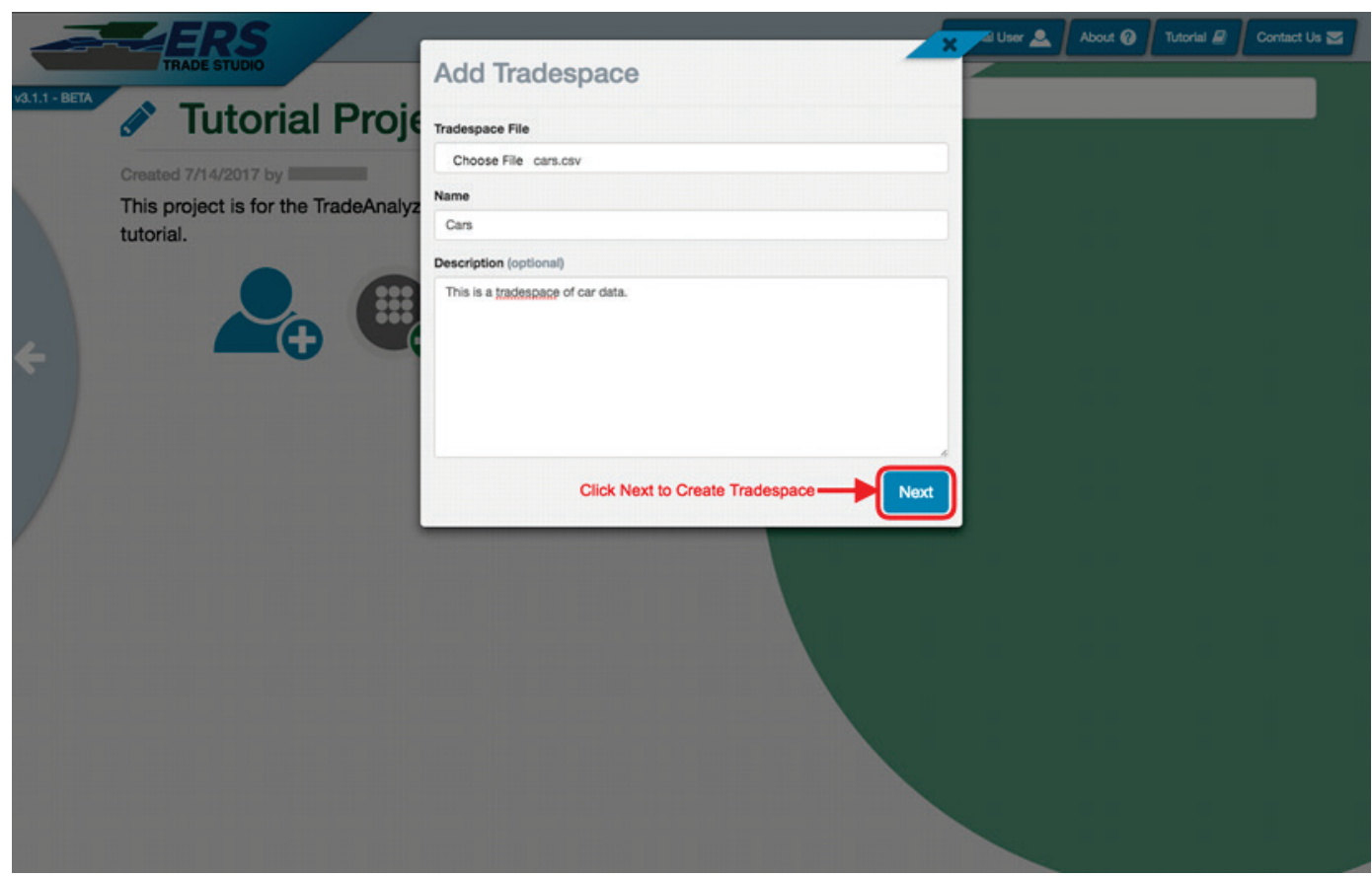

When uploading a CSV, a user can perform the following checks to make the process run smoothly:

- Ensure that all columns in your CSV have unique headings. Having duplicate or empty column headings will cause a loss of data on upload.

- Only use alpha-numeric symbols in headings and data values. Cells with special characters will get interpreted as strings, and sometimes cause errors on upload.

- Whenever possible, avoid using string data, as it is ignored in most of the visualization tools provided by TradeAnalyzer.

- Ensure that all cells have data. Empty cells will be removed on upload. Even though tools like Excel handle empty cells just fine, cells in TradeAnalyzer tradespaces are required to be complete.

- Avoid parameters with mixed types. This may cause the parameter to be interpreted as a string or cause errors on upload.

After selecting a CSV and naming the tradespace, the user should click the Next button in the Tradespace Upload modal, closing the modal and uploading the CSV. The tradespace is now represented by a translucent tradespace icon marked as "Pending" in the Project View (Figure 12). 
Figure 12. Pending tradespace.

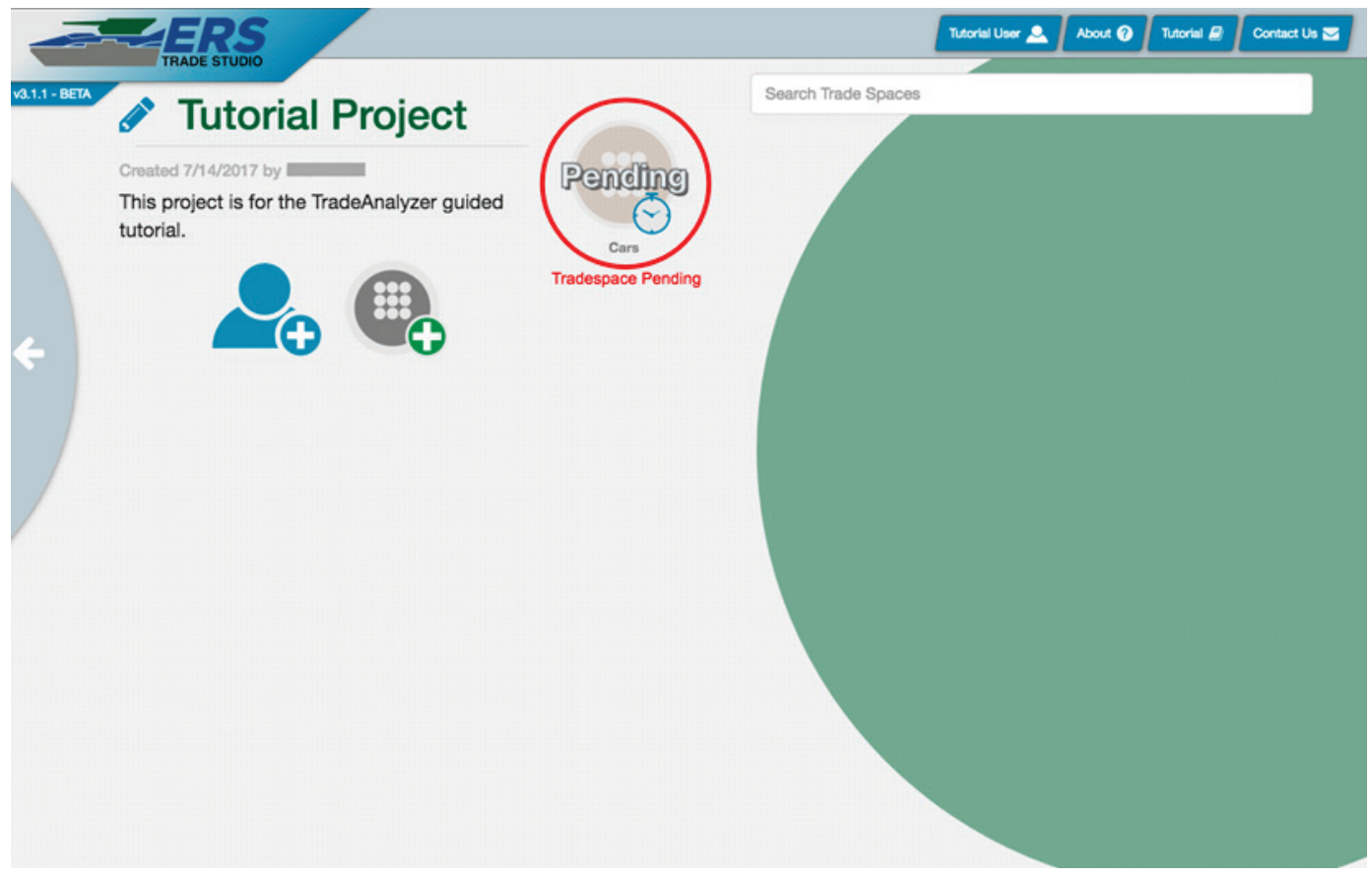

Once a CSV is uploaded, the server processes the tradespace data and precomputes statistics used in TradeAnalyzer tools. When the CSV is finished processing, it will have one of two results. If the data can be processed without dropping any columns or rows, it will automatically assign types to each column and show a complete and ready to view tradespace. The tradespace that is finished uploading has a number inside of the icon representing how many rows are in the dataset (Figure 13). 
Figure 13. Finished tradespace.

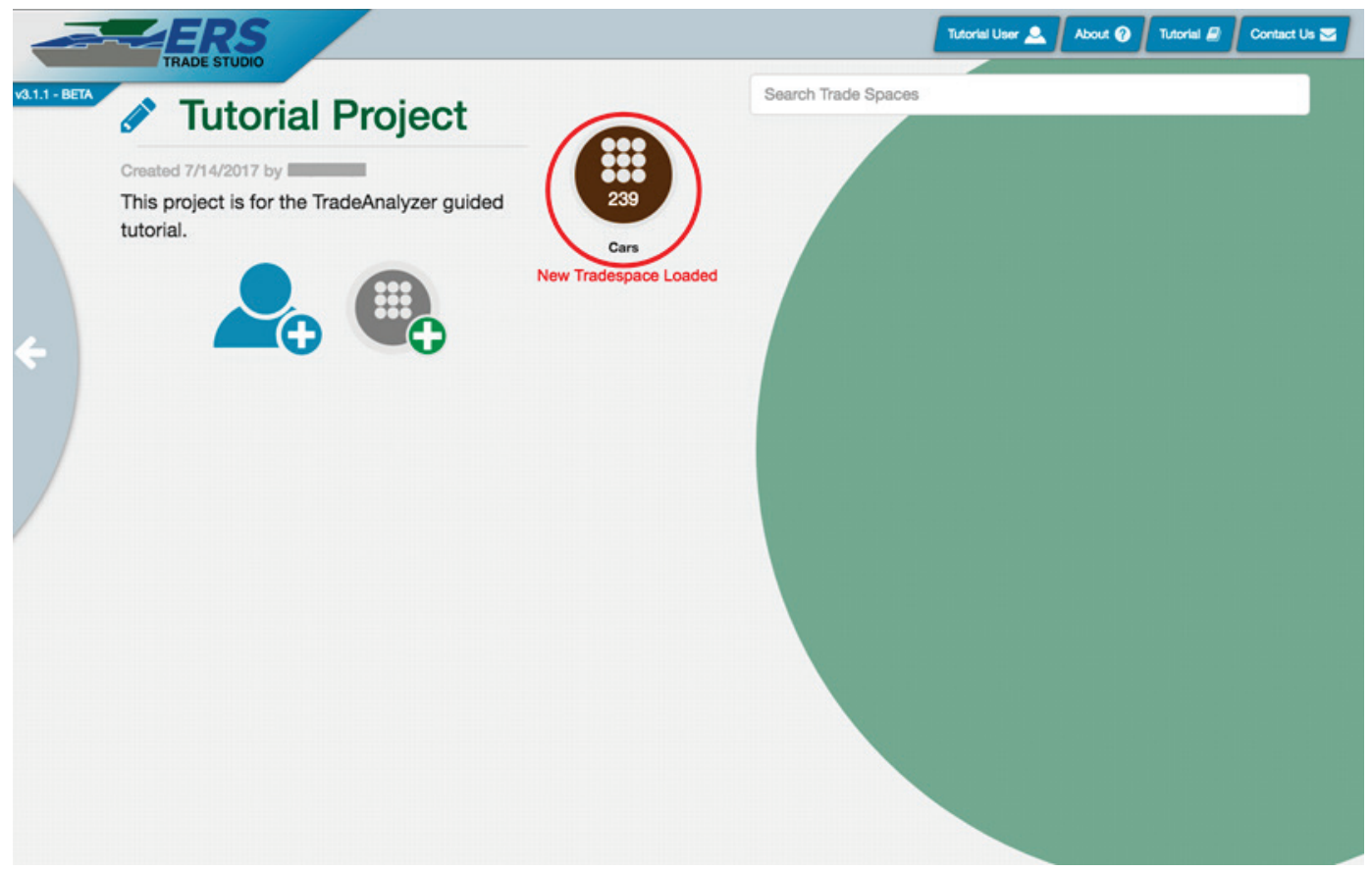

In the event that the server was unable to handle parts of the data, the tradespace icon will be presented with a small warning sign in the upper right portion of the tradespace icon (Figure 14). Handling a tradespace with errors is covered in the Data Cleaner section.

Figure 14. Tradespace with errors.

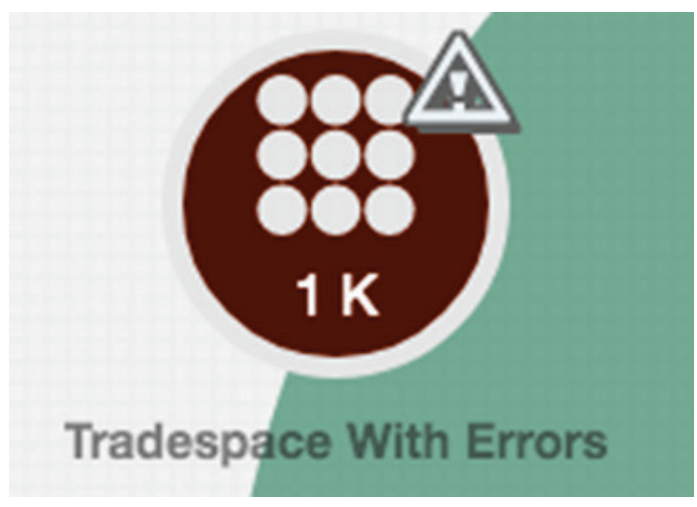




\section{Data Cleaner}

Some datasets will upload with errors. If the tradespace has an icon resembling the "Tradespace With Errors" page (Figure 15), then the data could not be processed without data loss. The data cleaner gives an opportunity to describe and properly interpret problem areas while maintaining the integrity of your original data.

Figure 15. Tradespace with errors.

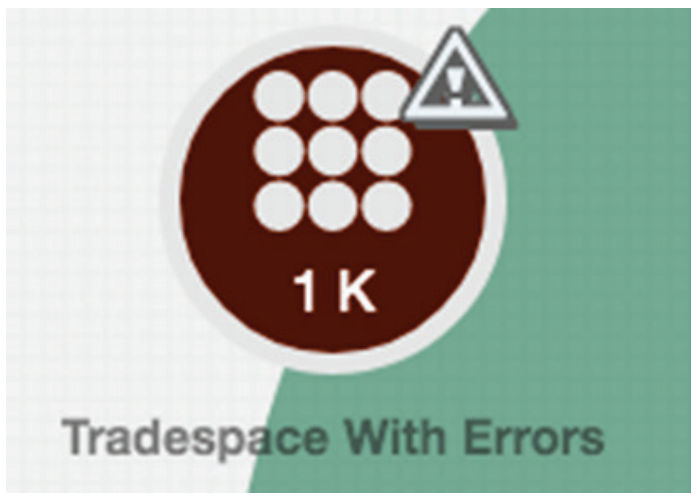

Clicking a Tradespace With Errors presents a modal (Figure 16) that displays a report of what the server failed to interpret. The errors are grouped based on their parameter. Clicking the + shows details about the errors, both what the values are and how many times it appeared. There are two options for tradespaces with errors; accept the data loss by clicking the Accept Errors button, or correct the errors manually by clicking the Clean Tradespace button. Accepting the tradespace will simply drop the rows with errors.

If a tradespace has too many errors, a user may find it best to edit the tradespace separately and upload a cleaned CSV. If the user wishes to delete the tradespace with errors, they should click the Clean Tradespace button. 
Figure 16. Data upload report.

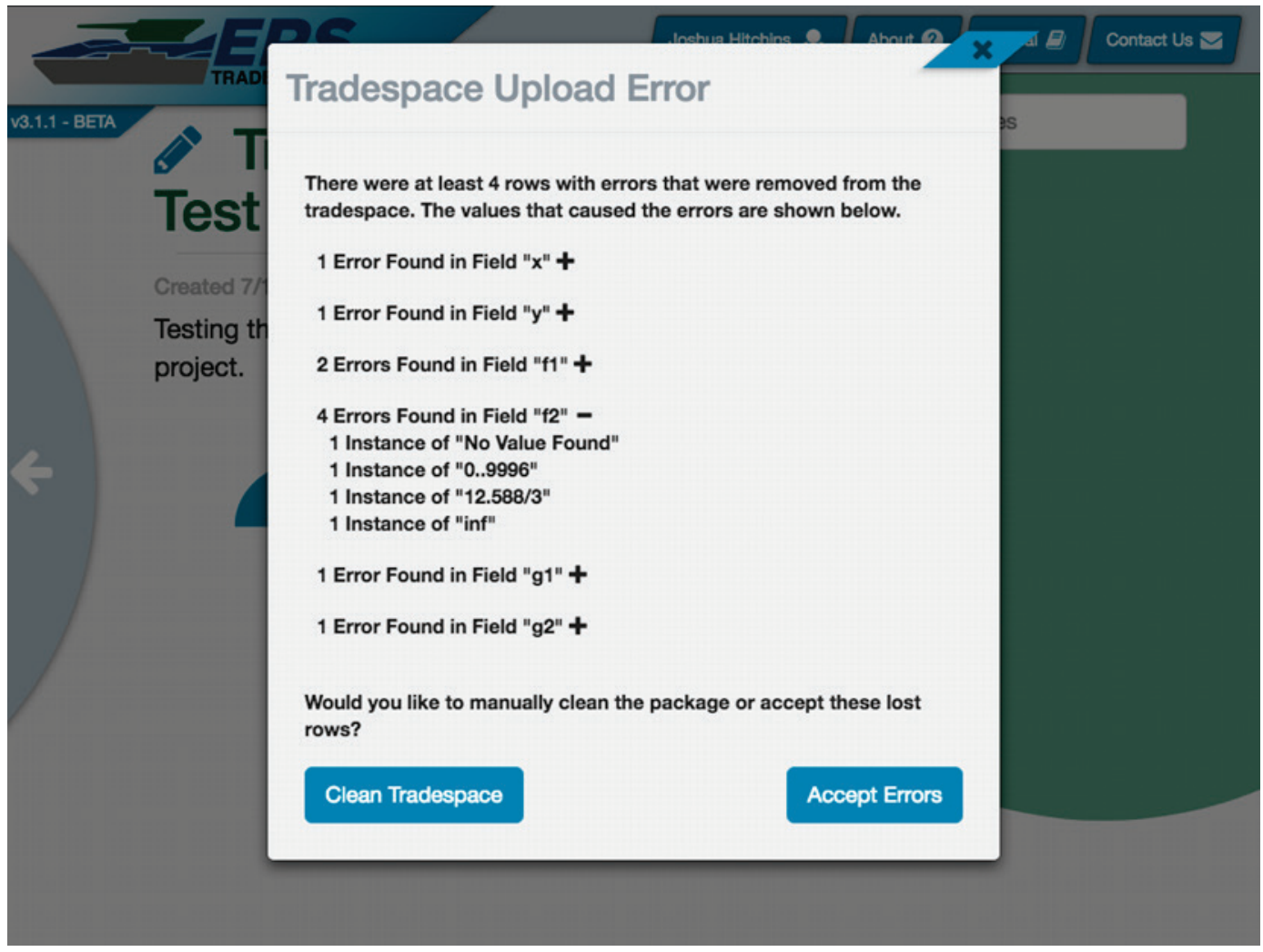

The initial view of the cleaner has the following functionality (Figure 17):

1. Set the type of parameters - The user can describe what type each parameter is from the following: integer, float, ordinal, or string. Columns with string values will not show up in most tools of TradeAnalyzer. However, the ordinal type can be useful for fields with a limited recurring set of string values, for example, days of the week. These values can be enumerated and graphed alongside other data.

2. Change the settings on upload - The user can modify how the CSV file is loaded, such as changing the delimiting characters (if the file is delimited by colons or semicolons for example).

3. Cancel the upload - If you do not wish to use the new tradespace, the user can remove the tradespace by clicking the trashcan icon at the top right portion of the tool.

4. Start upload - Clicking the "Check Values" button initiates the next step in the CSV file upload. 
Figure 17. Data cleaner initial view.

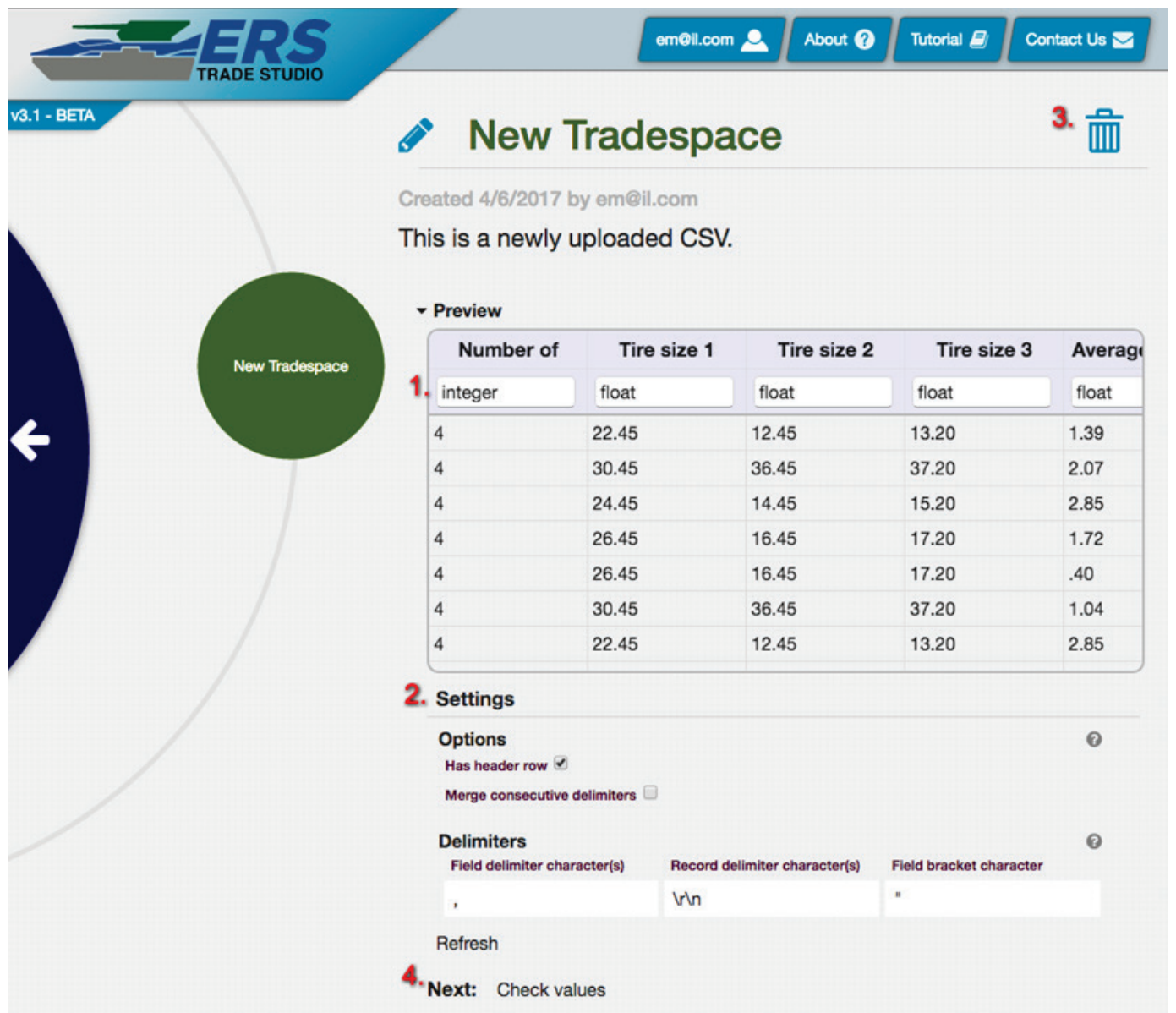

Once finished with the initial view, the server scans the tradespace for any incorrect or missing values. The unhandled values view allows the user to determine how the CSV file will be corrected.

The data cleaner's unhandled value view has following functionality (Figure 18).

1. Remove Parameter - If a parameter has too many unhandled values or does not contain useful data, the user has the option to remove it.

2. See unhandled values - Clicking the flag brings up the unhandled values on the right side (labeled 6).

3. Search parameter - Clicking this does the same as clicking the flag, but it will not show any unhandled values, as there are none in that parameter.

4. Remove row - A user may choose to remove a row if they feel it is there erroneously, has incorrect data, or it is not useful.

5. Search for all values - Searching in this bar will search for all values in this field matching the input. 
6. Correct unhandled values - These unhandled values can be clicked to correct them. A user may change the value (or all values matching that type) to be valid, remove the offending row, or remove the column.

7. See existing policies - If a user chooses to change the data, the policies will be listed here. If they wish to revert their changes, there will be a trashcan to allow deletion.

8. Finalize changes - When the user is finished editing the data, click Create Tradespace to generate the tradespace.

Figure 18. Data cleaner unhandled values.

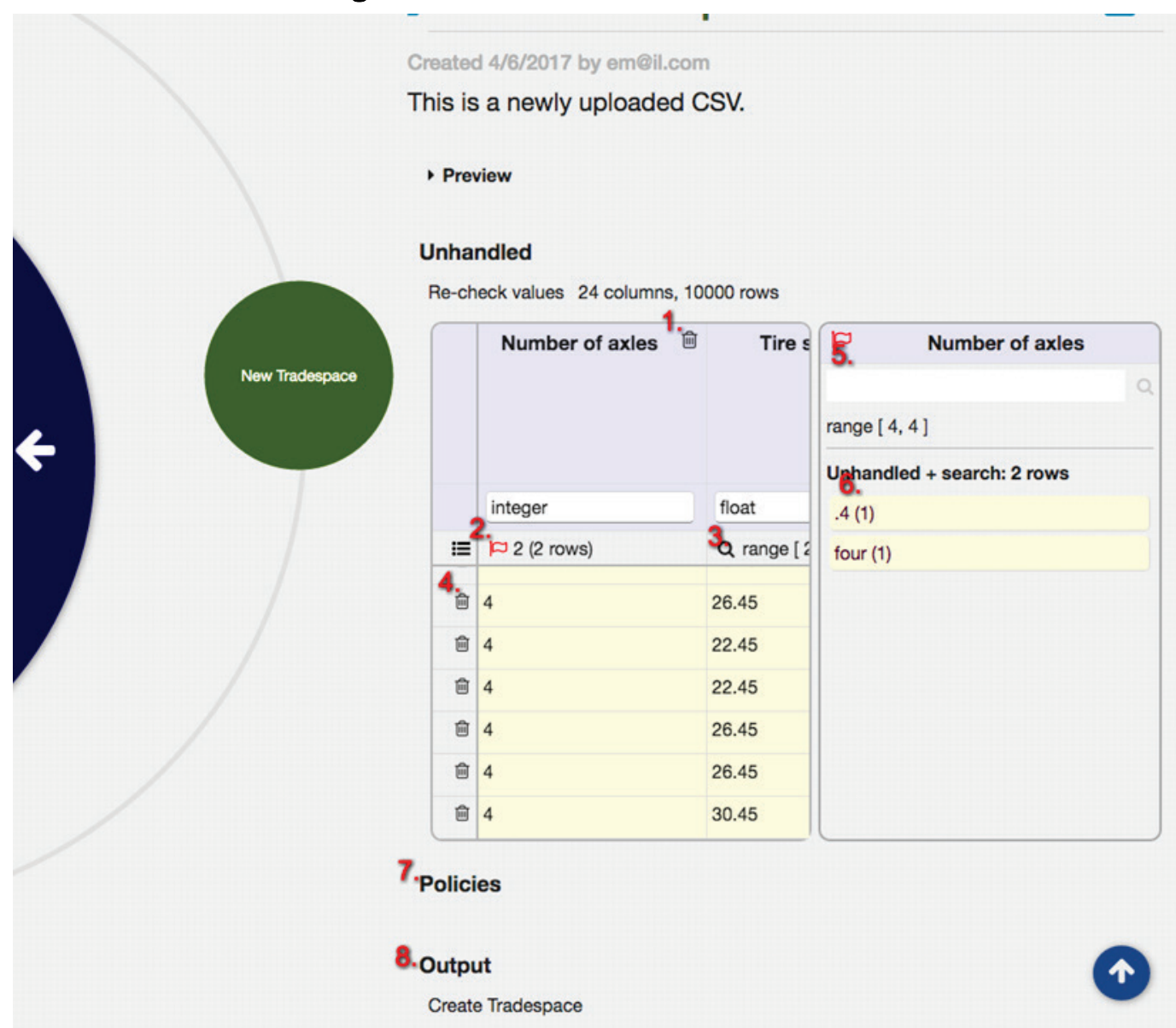

There are features to edit specific value. To enable the value editor, the user should click on the red flag above a column with errors (label 2 in Figure 18). This will hide all but the problem rows in that column and begin editing inside of the first one. There are three new icons available when editing a value (Figure 19):

1. Accept - The check icon accepts the edited value in specifically that cell. 
2. Edited - The check with arrows pointed up and down accepts the edited value in all error cells in this column. This is for editing bulk problems like null values.

3. Trashcan - The trashcan icon deletes all of the rows with that error.

All changes will be reflected in the Policies section of the tool and can easily be reverted by removing the desired policy.

Figure 19. Data cleaner editing values.

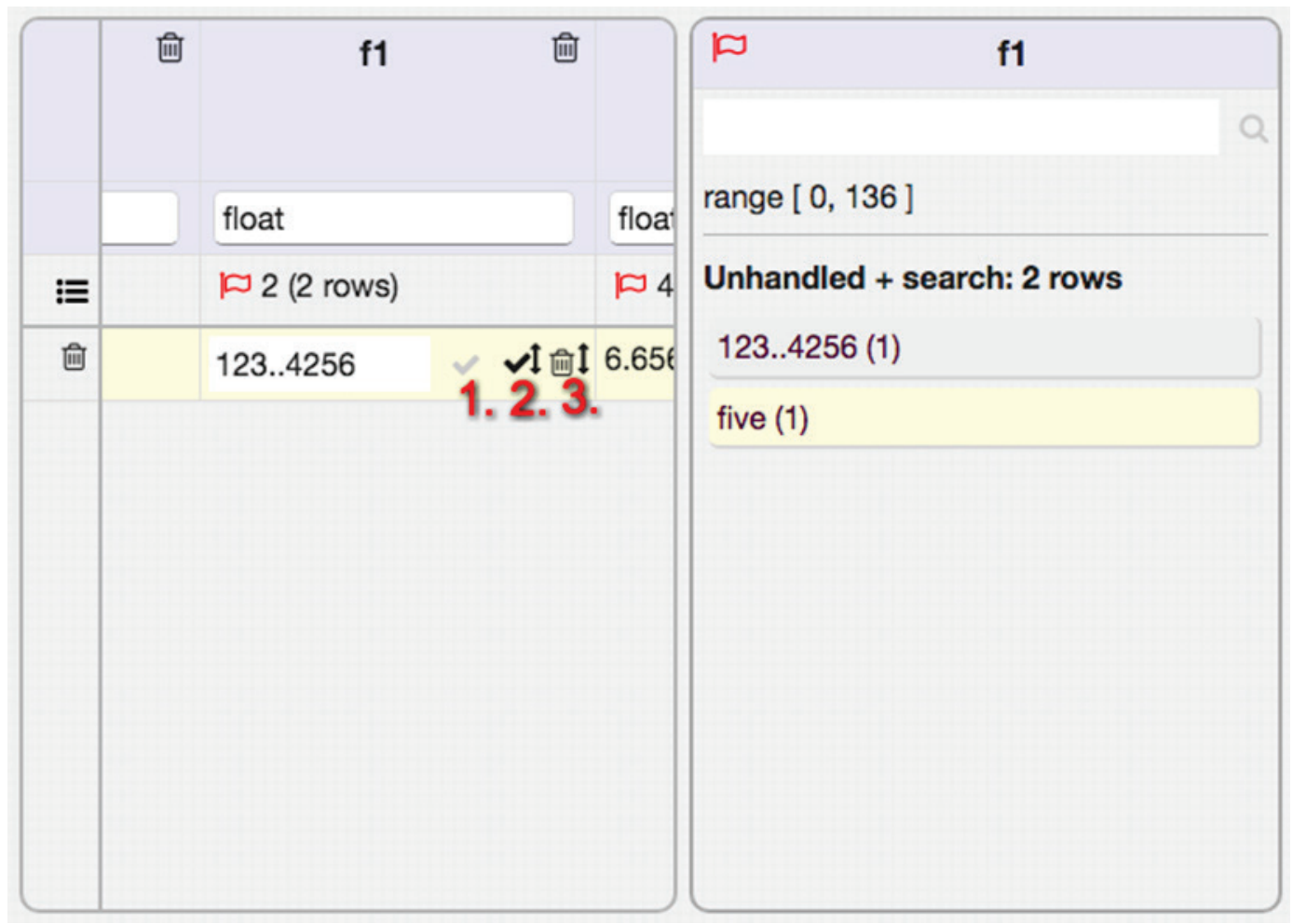

Once finished setting up the change policies, the user can finish cleaning by clicking the Create Tradespace button. Note: once the tradespace has been generated, a user cannot edit or delete a tradespace with the Data Cleaner and must upload a new CSV. This is by design, as multiple users may be relying on the data to be consistent. 


\section{Tradespace View}

The tradespace view contains a number of tools that can be used to visualize and analyze the tradespace. These tools will allow a user to create subsets, snapshots, and script results. More information about these tools will be covered in later sections. As seen in Figure 20, no subsets, snapshots, or script results are included in the tradespace by default.

Figure 20. Tradespace view.

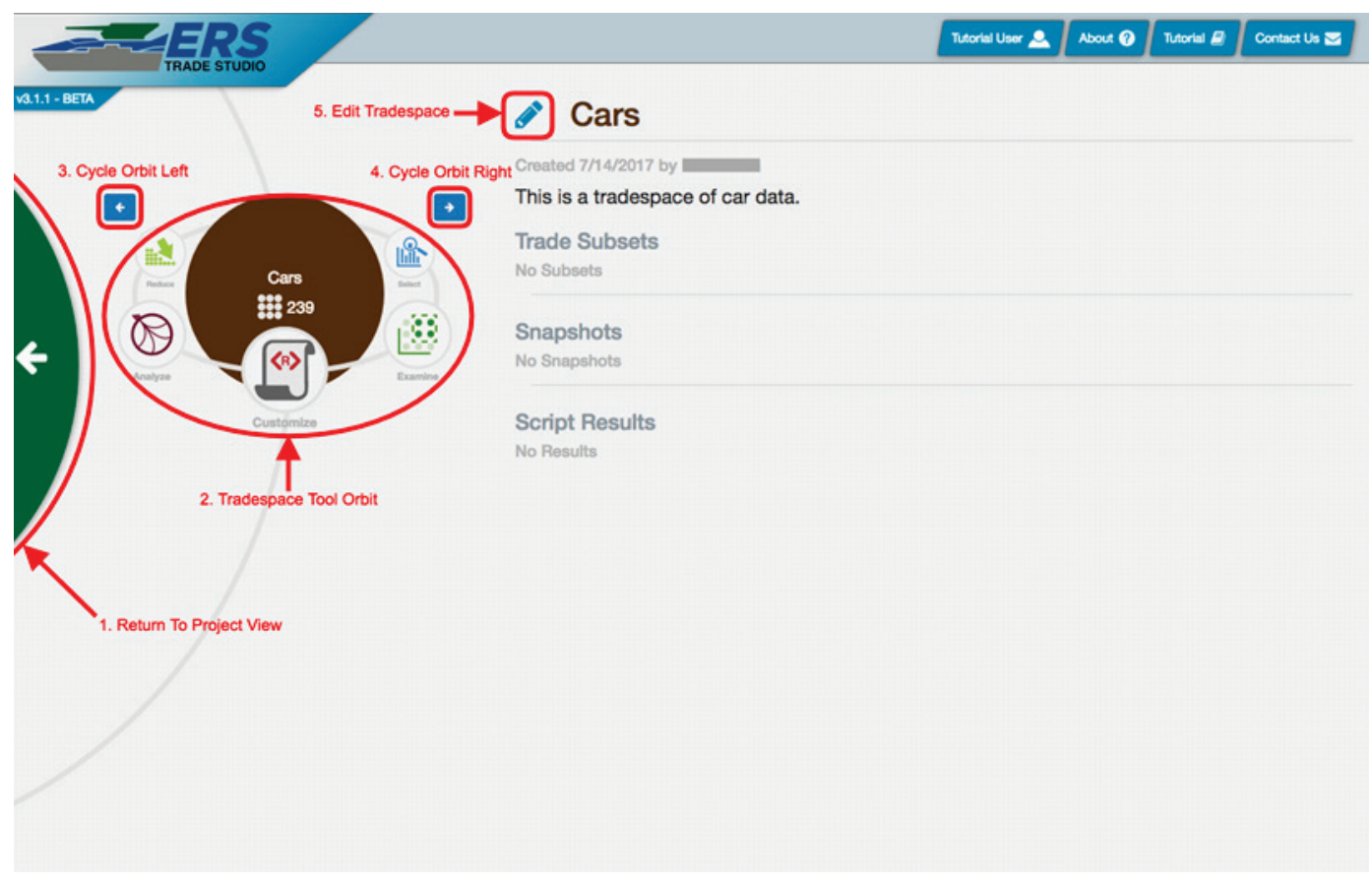

The button on the left side of the screen returns the user to the Project View. When inside any tools, the button returns the user to the Tradespace View.

The tool orbit contains all of the tools on the page. These tools exist to refine, analyze, and visualize the tradespace. Each tool will be explained further in the guide in their respective sections. The arrows to the upper left and right of the tool orbit cycles through the available tools.

Clicking the pencil next to the tradespace name presents the user with the Edit Tradespace Modal that modifies the name and description of the tradespace. Users who are Guests on the project will not be allowed to use this feature. 
Any subsets to the tradespace appear below the name and description (Figure 21). Clicking on a subset goes to its own Tradespace View. Trade subsets function exactly as normal tradespaces.

Figure 21. Tradespace view with subset.

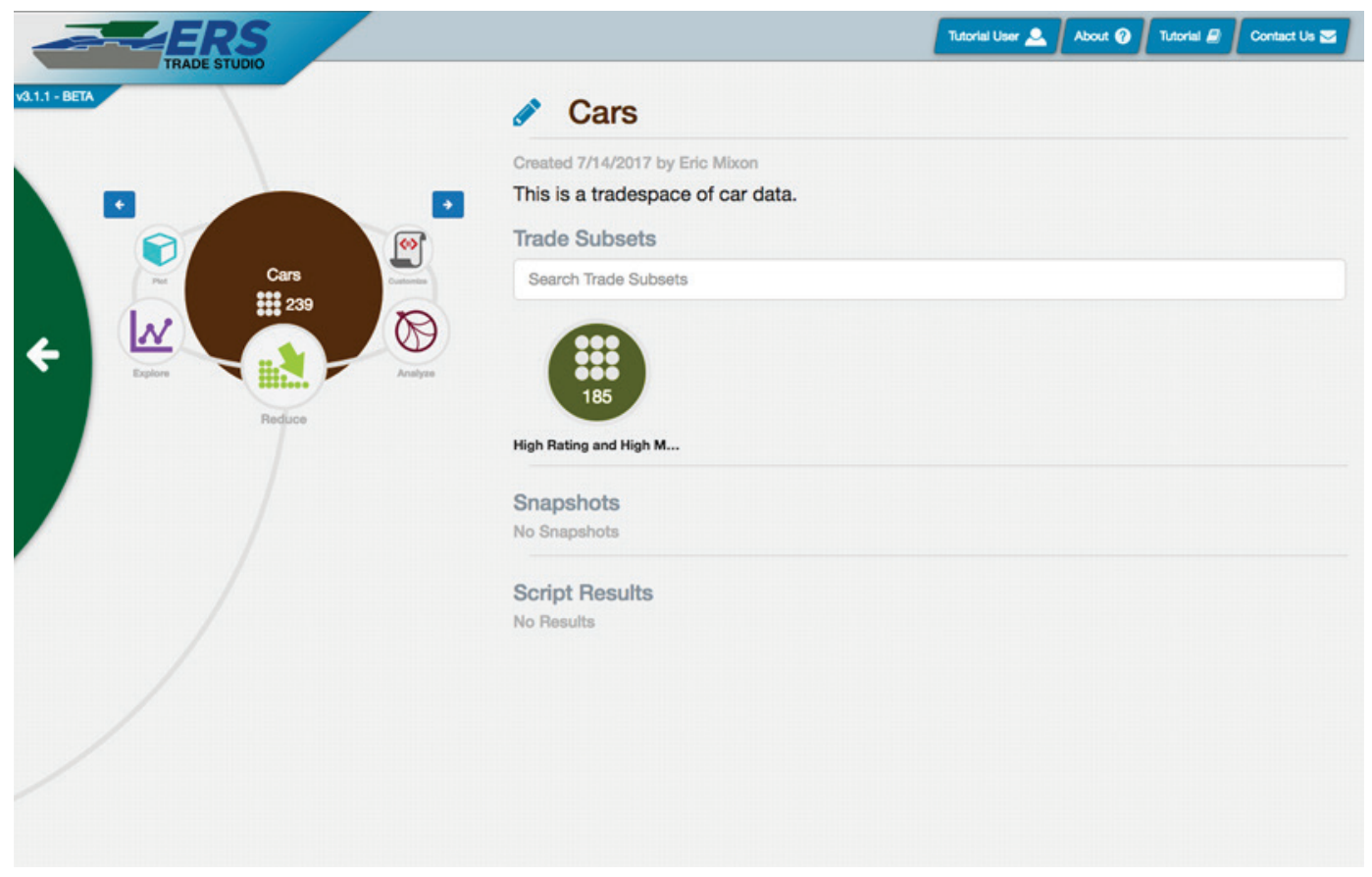

When opening trade subsets, the tradespace view shows the path to the current tradespace as a series of icons along the left side (Figure 22). The user can navigate back up the chain by clicking any of the icons or using the up arrow. 
Figure 22. Tradespace view with subset selected.

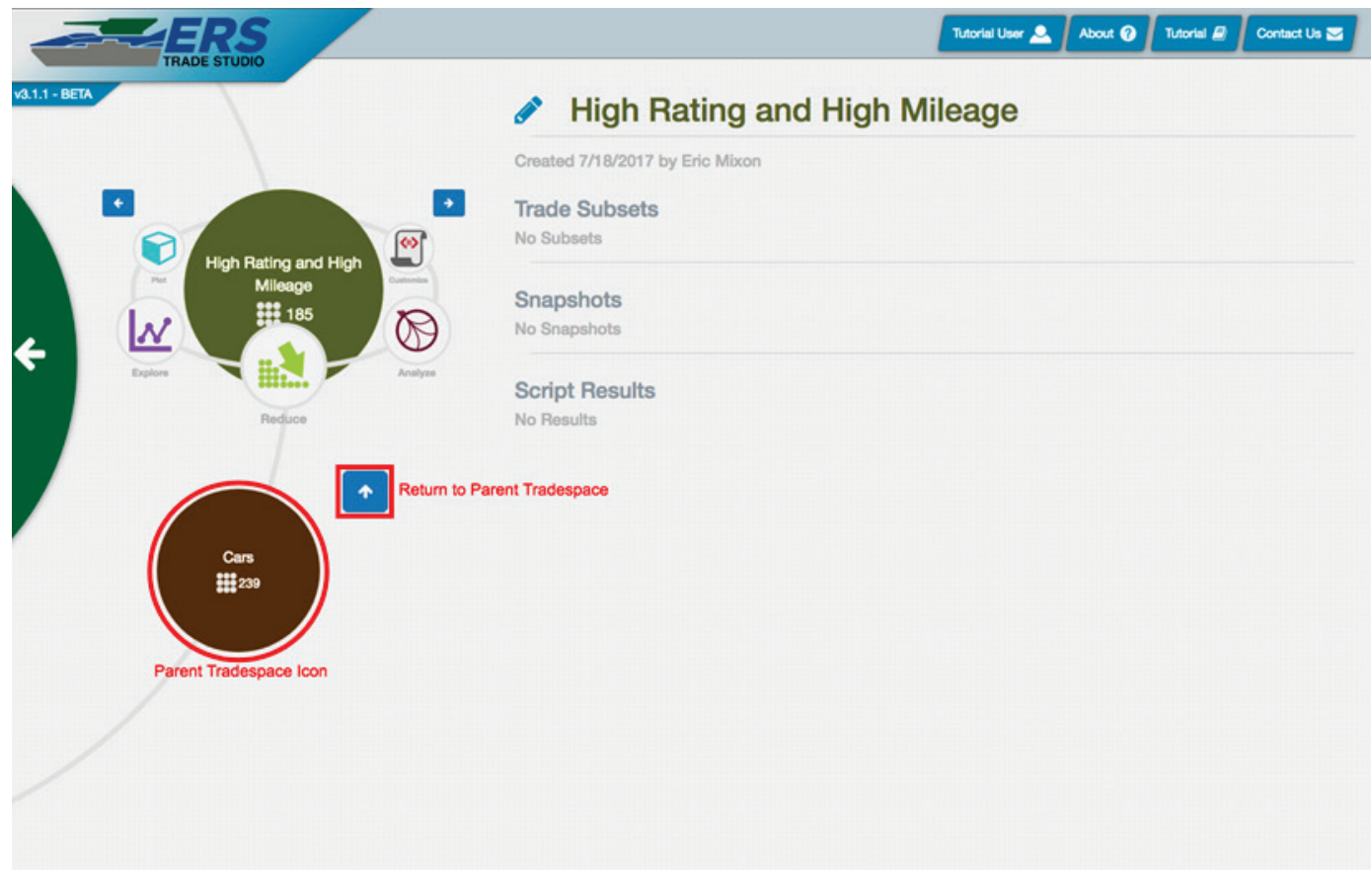

Snapshots contain specific information that has been saved from a tradespace analysis. Snapshots appear in their own section below the name and description (Figure 23). Clicking a snapshot loads a previously saved state. This will include saved objectives and data selections. These topics will be covered more thoroughly in later chapters.

Figure 23. Tradespace view with snapshot.

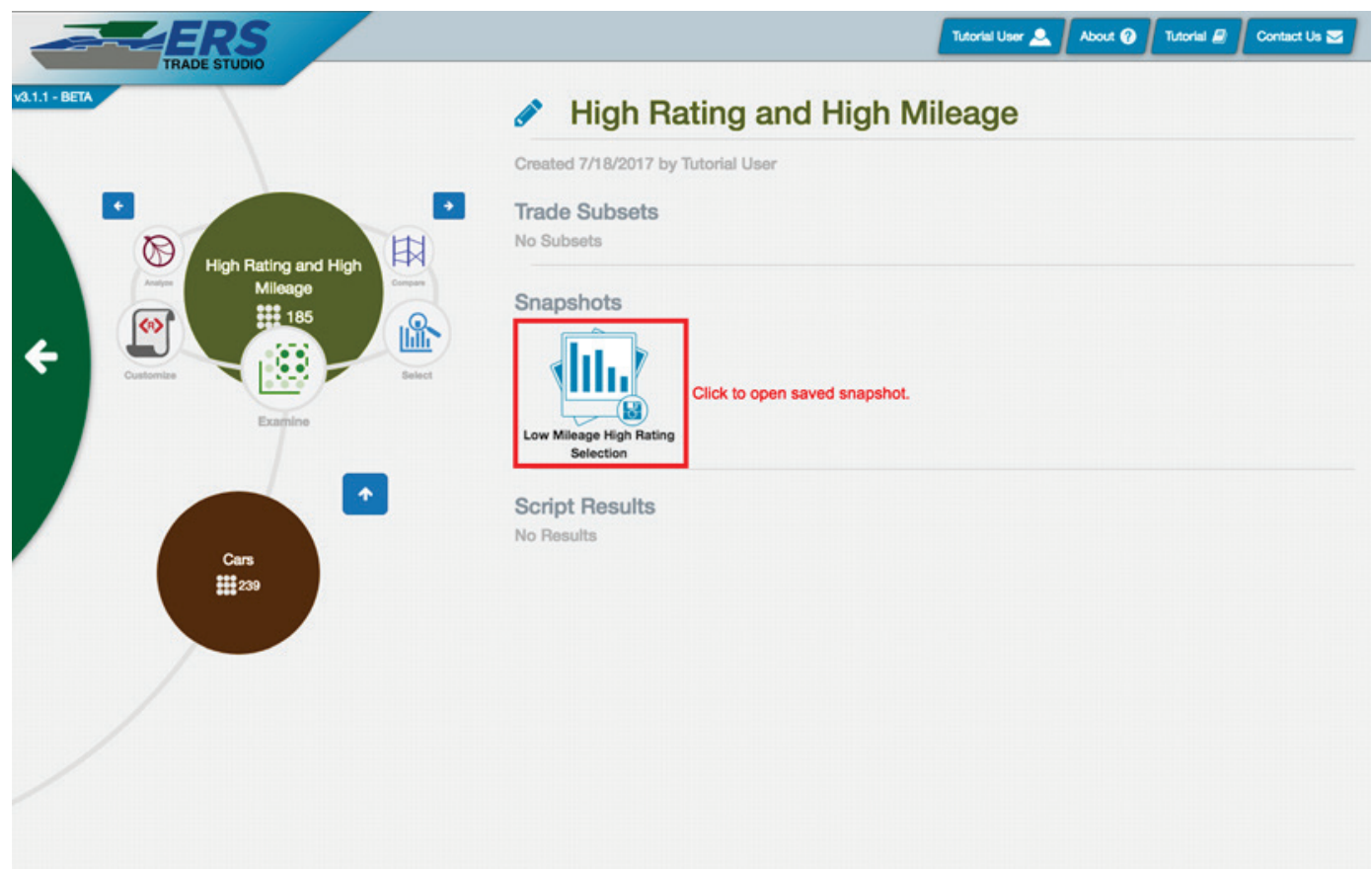


Script results produced by custom written and uploaded R Scripts are displayed below the snapshots (Figure 24). Clicking on a result will display open the result images or display information about trade subsets that may have been generated by the script.

Figure 24. Tradespace view with script result.

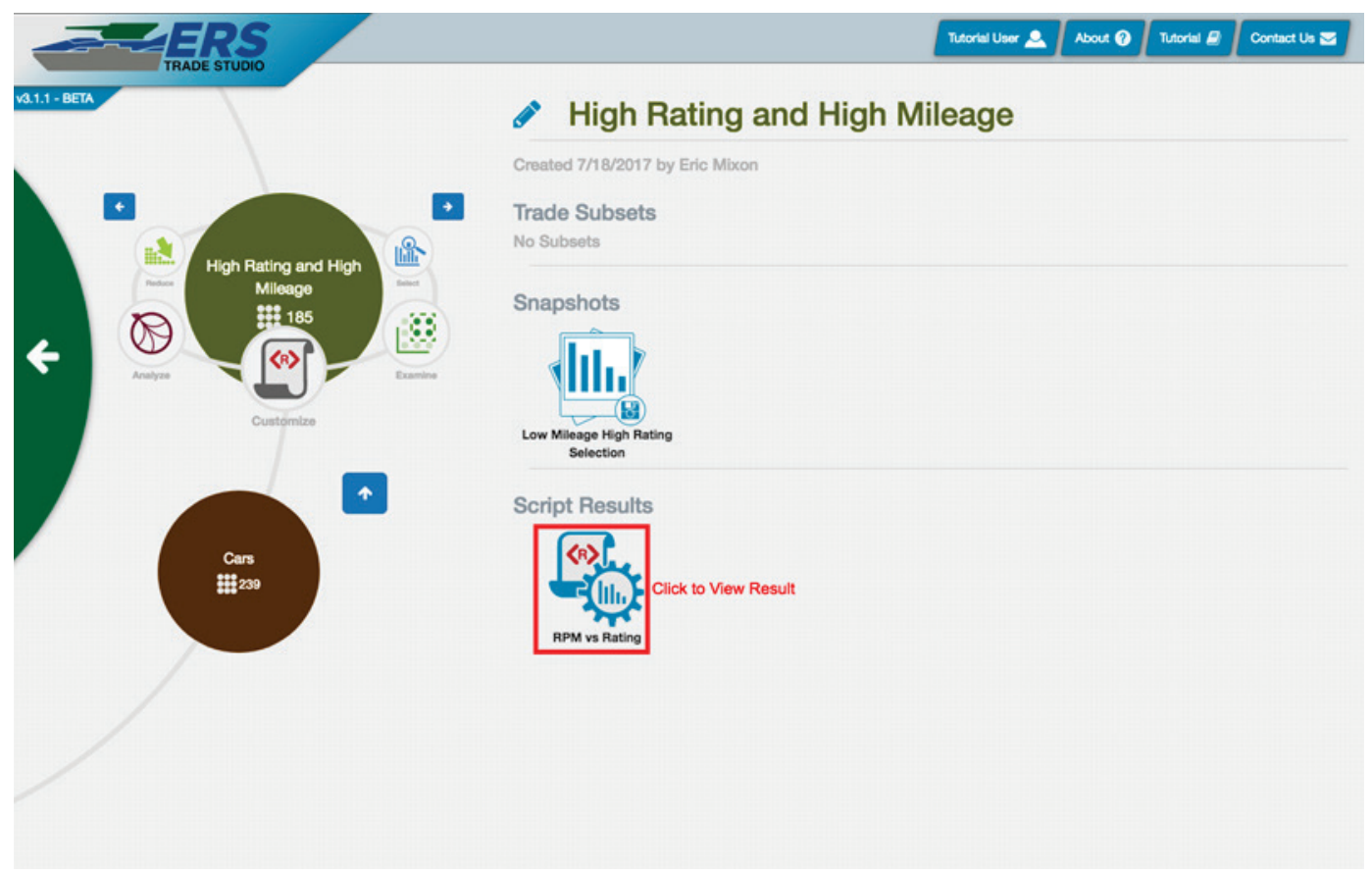




\section{Parameters}

Parameters are aspects of your points that differ from point to point. If the points are rows in your data set, parameters are columns. Many tools show comparisons between points along one parameter, while others show how parameters relate to other parameters.

Figure 25. Parameter selector.

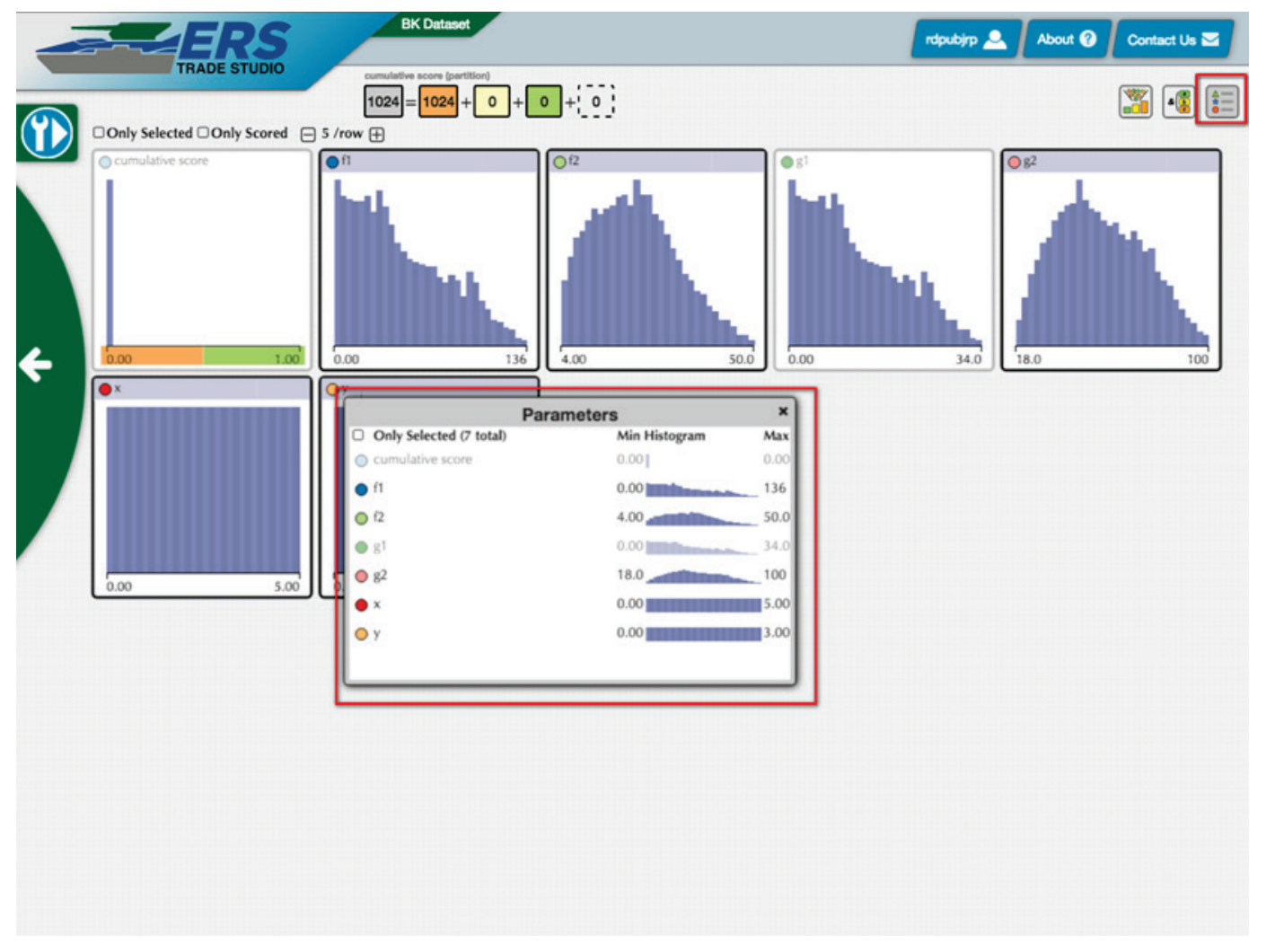

The parameter selector, outlined in red in the above screenshot, is a tool for selecting and deselecting parameters. This tool is available in many views, allowing the user to visualizations only the selected parameters. The user can choose to list only the selected parameters by clicking the checkbox label Only Selected in the top left of the parameter selector. Also, the parameter selector shows histograms of the parameters along with the minimum and maximum values.

A user can access the parameter selector by selecting the parameter selector icon outlined in red in the top right of the screenshot above. A user can exit the parameter selector by clicking the $x$ at the top right of the parameters box. 


\section{Annotations}

Annotations are a way to rate points based on desired parameter values. There are two different types of annotations, partition annotations and range annotations. Both types of annotations can be fine-tuned in the Annotation Editor, this is accessed by clicking the icon as shown in Figure 26.

Figure 26. Annotation Editor icon.

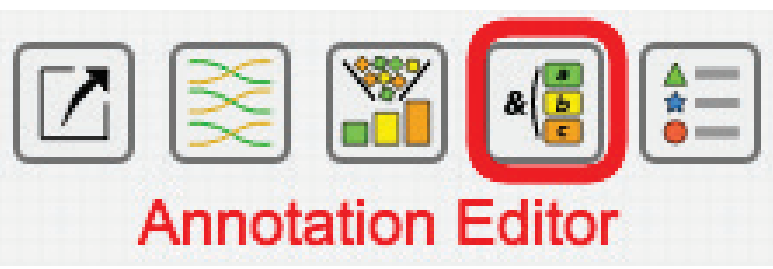

This section will focus on the Annotation Editor's functionality and annotation editing capabilities in the Reduce and Compare tools. Further explanation of the Reduce and Compare tool's functionality are in their respective sections.

Partition annotations are created using the Reduce tool's histograms and are limited to an individual parameter. These have different partitions selected as yes, no, and maybe, represented by green, orange, and yellow, respectively. If a point falls into a yes category, it receives a positive score equal to the weight given to the annotation. If a point falls into a no category, it receives a negative score. If it falls into a maybe category, it receives no score.

Uncertainty can be added to any boundary between two categories. Any point falling in the uncertainty area receives a score between the two scores, with the boundary itself getting the average of the neighboring scores.

In the Reduce tool, clicking the star in the top right of a histogram creates a partition annotation. This should select the entire parameter as a maybe (yellow) category. Clicking on the histogram will change the category of the partition and add a divider, splitting the current partition into two (Figure 27). Double-clicking a divider edits the uncertainty and the value. Any non-active annotations are shown below the respective parameter's histogram, while active annotations will cover the entire histogram. 
Figure 27. Adding a divider.

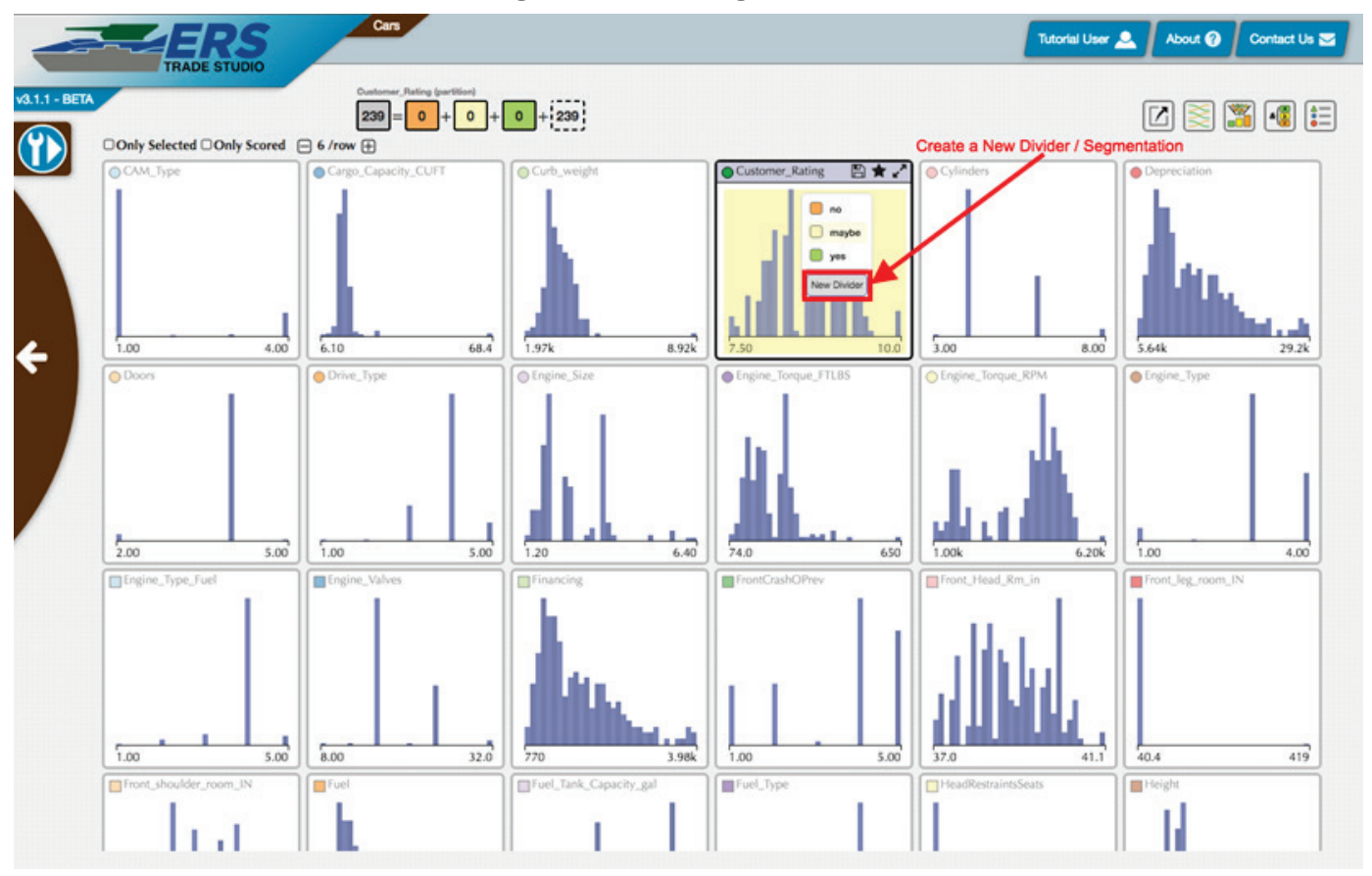

The first time an annotation is created, the Annotation Editor will open (Figure 28). This allows the user to name the partition, set exact values for the divider, adjust the weight, set uncertainty, set a rationale for the annotation, and save the annotation. The Annotation Editor only edits the active annotation and the last parameter edited. The active annotation's name appears at the center top of the screen over the grey box showing the total number of points.

The name and rationale can be changed to help identify annotations. Annotations can be switched by using the list of annotations on the left side of the Annotation Editor. Annotations can be edited and saved by clicking Update, saved as a new annotation with Save As New, revert to the old save with Revert, and cleared by clicking Reset. 
Figure 28. Annotation Editor - partition.

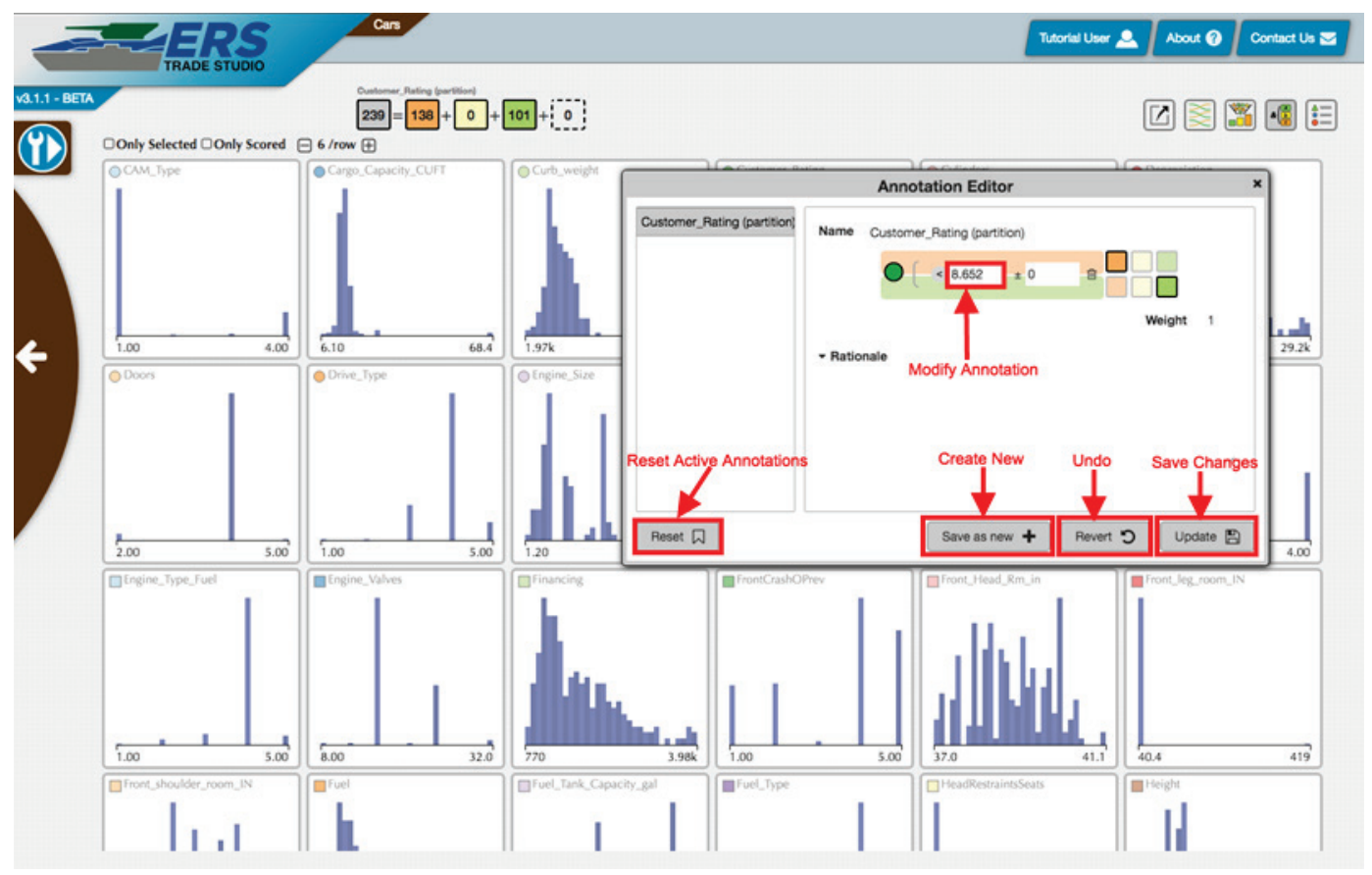

The other kind of annotation is a range annotation, this is created in the Compare tool. Unlike the partition selection, the range annotation can only have two categories, the points that fall within all of the ranges provided and those who do not. However, the range selection can be across different parameters.

The Annotation Editor is different for range annotations (Figure 29). There is no uncertainty in range annotations, the user is only either within the range, or not. Points within the range receive the appropriate score (positive weight for yes, o for maybe, and negative weight for no), while points outside of the selection receive no score. Other functionality, such as name and rationale changing, saving, and updating, is the same as the partition annotation.

The symbols \& and // in the range annotation represent what points are within the annotation. The // symbol means the points can fall in either category, usually on the same parameter. The \& symbol means the point must fall in both ranges. These are usually different parameters. 
Figure 29. Annotation Editor - range.

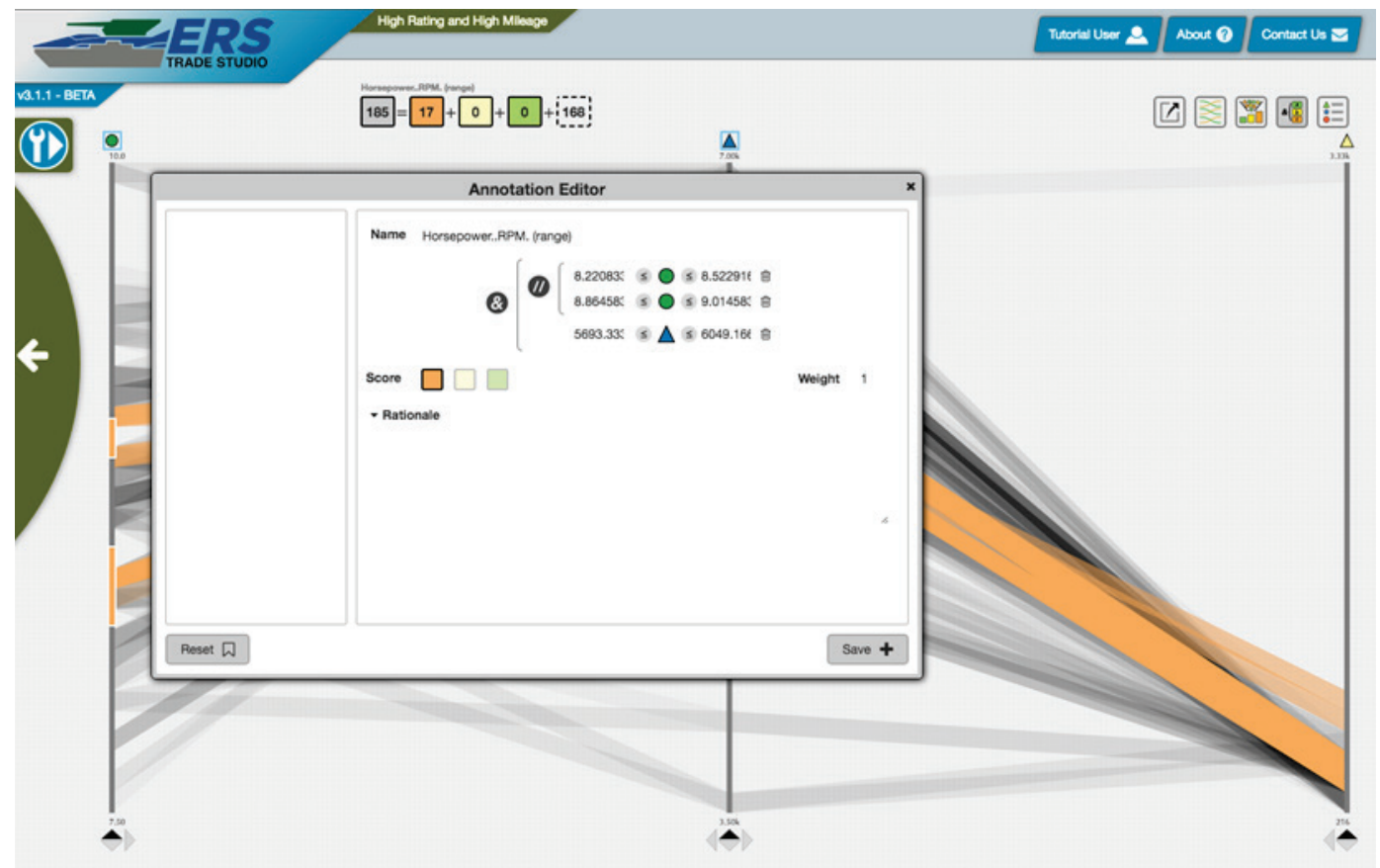




\section{Objective}

Objective is defined as a combination of annotations. Objective allows for reducing data and creating complex parameters to compare points. The user may access the Objective Function Builder (Figure 30) by clicking the icon in the top right in the Combine, Plot, Analyze, Reduce, and Compare tools. This creates and visualizes objectives.

Figure 30. Objective Function Icon.

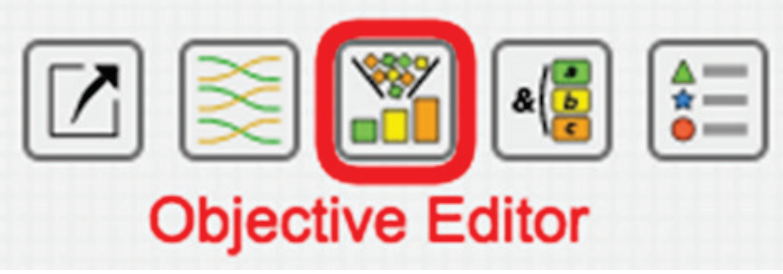

In order to build your objective, there must be saved annotations.

Selecting annotations via the dropdown or via typing their name adds them to the objective, as shown in Figure 31. Once all of the desired annotations are selected, clicking Save as New saves a new objective. This will create a histogram showing the distribution of your scores.

Figure 31. Objective Function Builder.

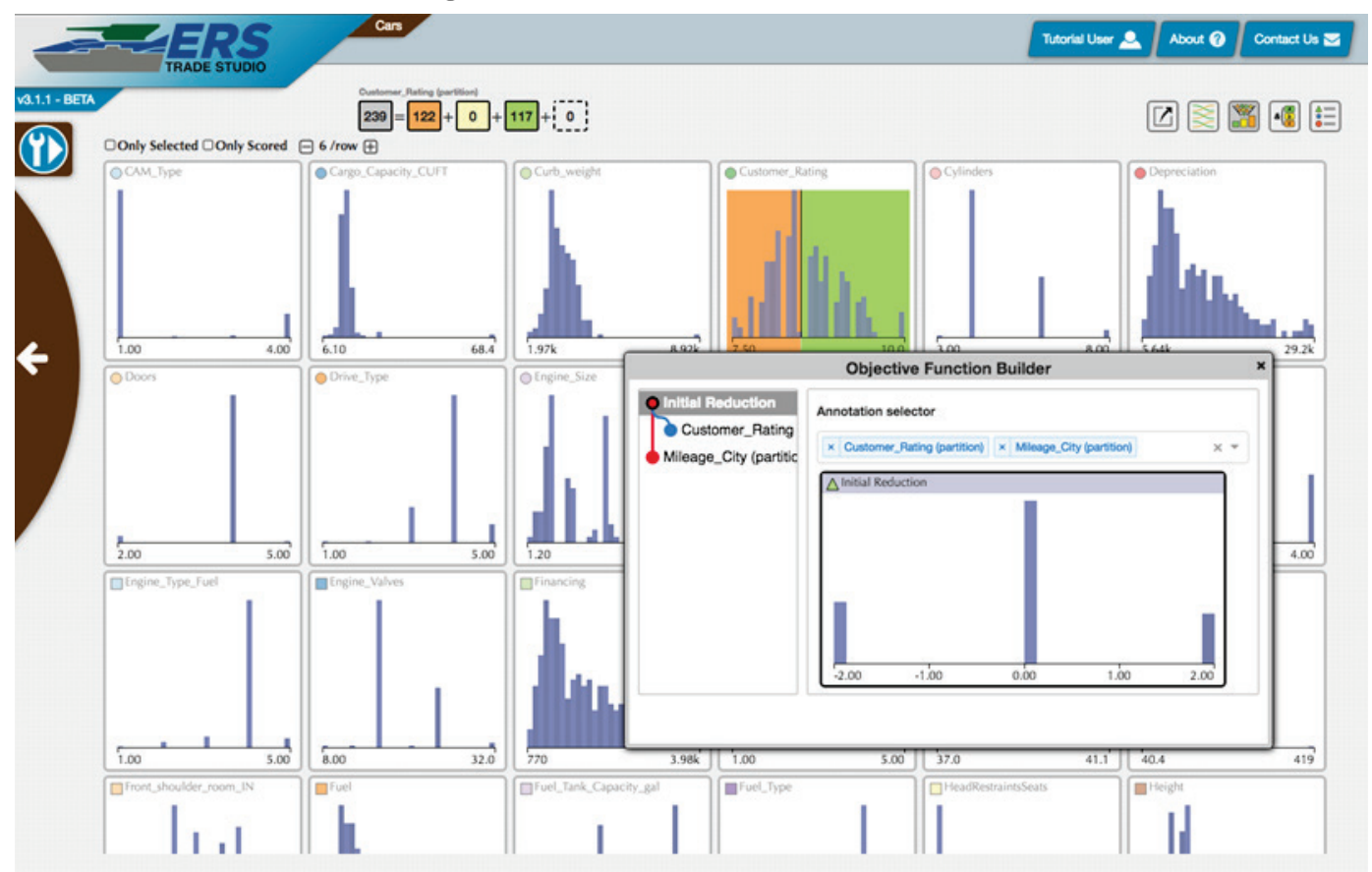


Once created, the Objective Function will operate as a parameter in the different tools. For instance, in the Plot tool, the point's size can be relative to their score. This allows you to view which parts of the tradespace are desirable. Objectives can even be added to annotations, creating multitiered objective functions.

Saved objectives and their respective annotations can be selected on the left side of the Objective Function Builder. Changing the underlying annotations or adding new annotations will edit existing objectives. Clicking Update saves any changes to the selected objective. 


\section{Export}

The Export function allows the user to create a subset of a tradespace. This function can be accessed from the top right corner of the Reduce, Analyze, Compare, Combine, and Plot tools.

Figure 32. Export Button Location

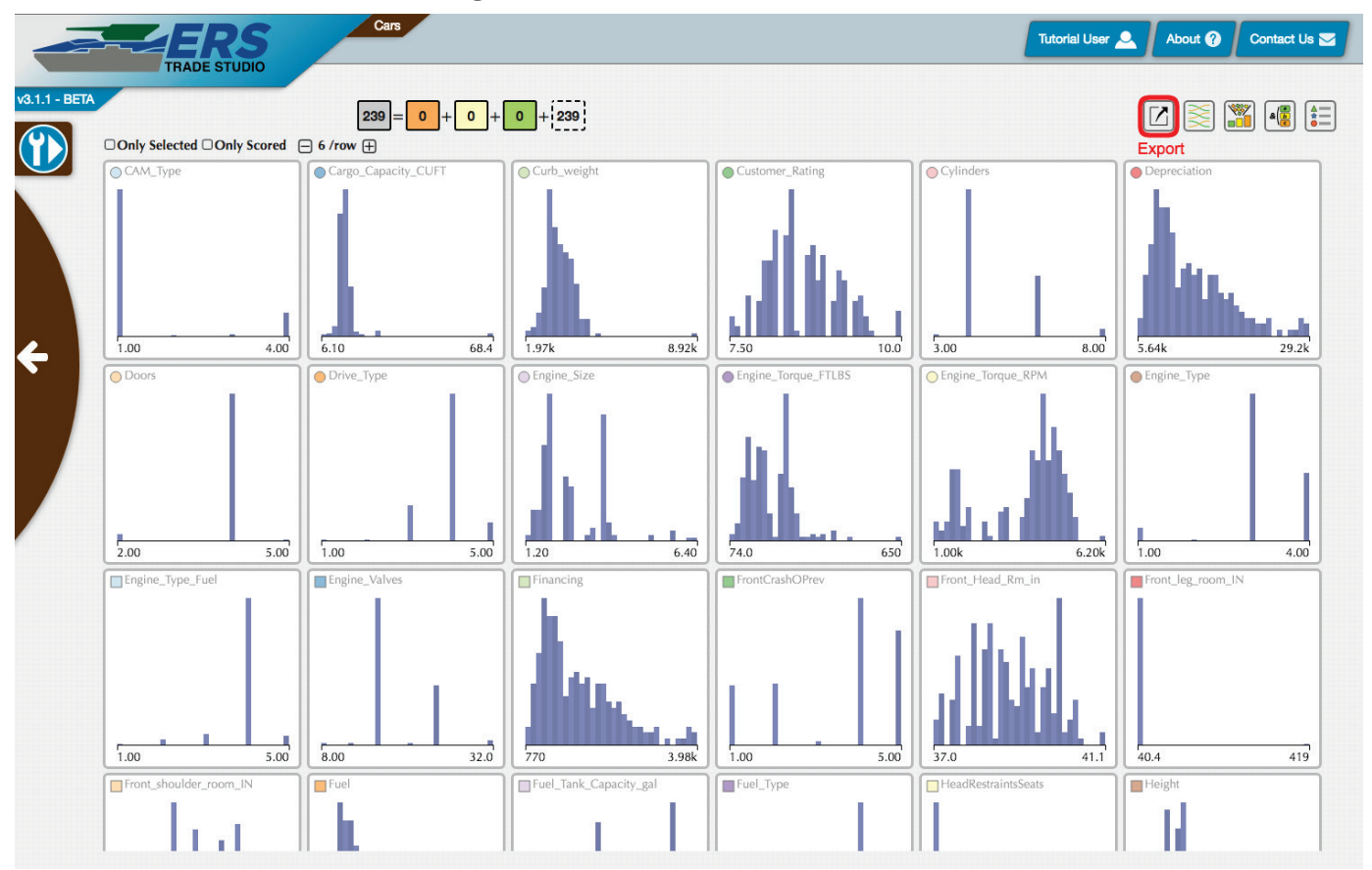

Once the user clicks the button, they will be presented with the export package view. 
Figure 33. Export Package View.

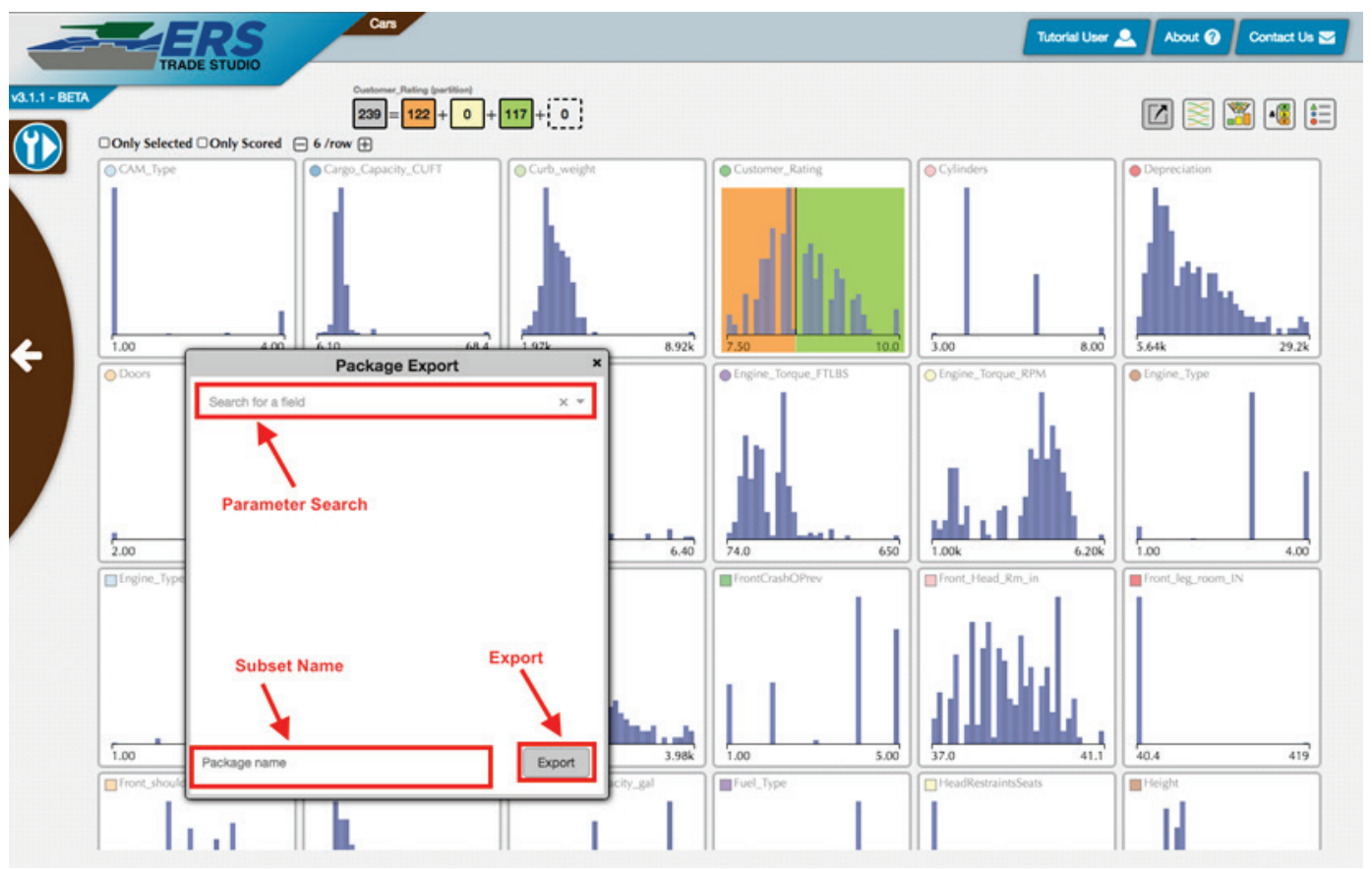

The following is a list of features within the package export view:

- Parameter Search - Allows the user to search for a specific field within the selected tradespace. This can be done by typing in a field name or using the drop down arrow on the right side of the search bar.

- Subset Name - Allows the user to name the subset. The user must provide a name before exporting.

- Export - Exports the selected data. The subset will show up in the tradespace orbit.

When the user selects data from the subset while in the package export view, the annotations view will appear with the option to further customize the subset. For more information about annotations, refer to the Annotations section. 
Figure 34. Export to Tradespace Subset.

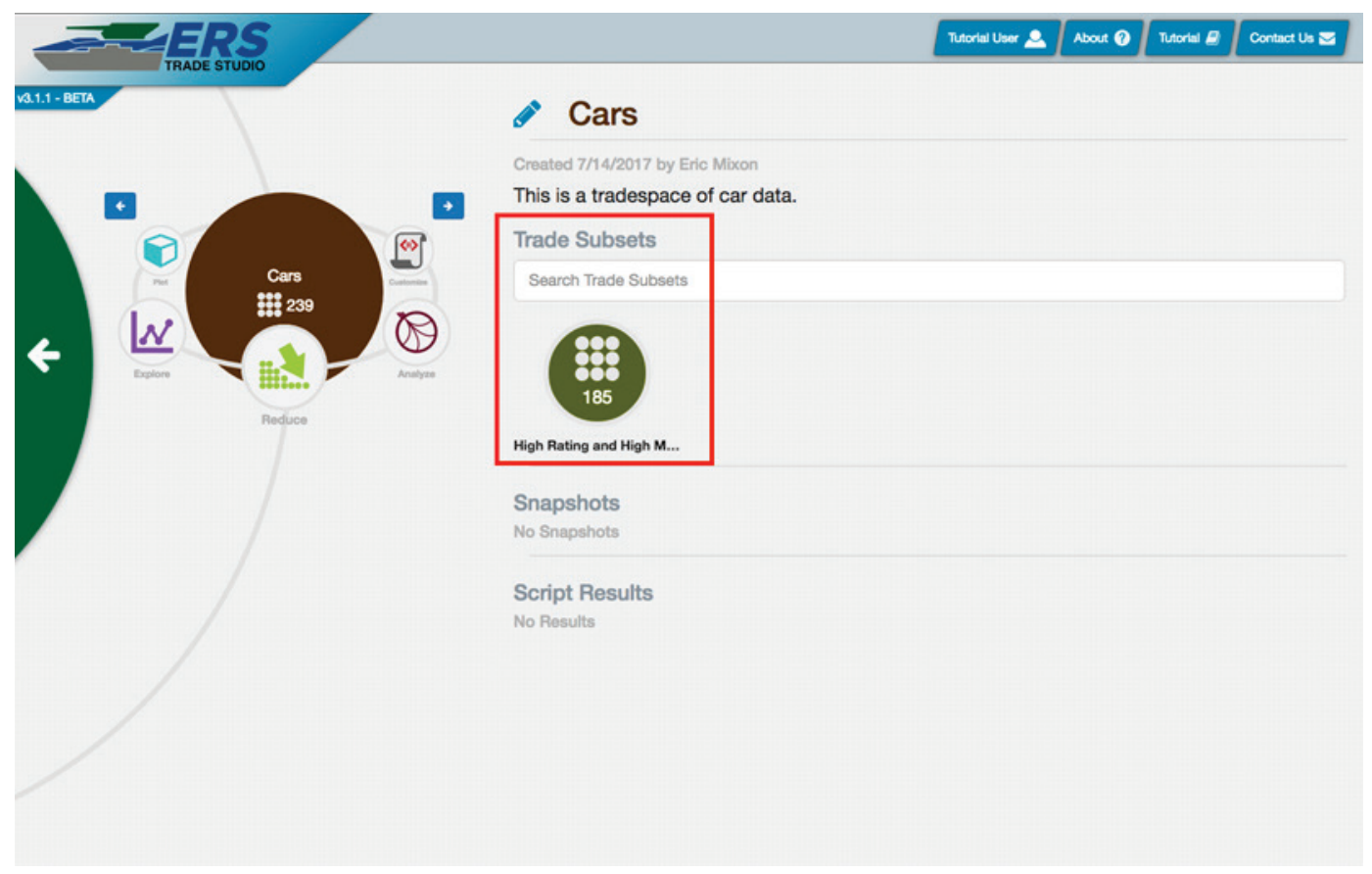

A subset can also be created within the Customize Tool. Additional information can be found in the Customize Tool section. 


\section{Compare Tool}

The Compare Tool helps compare parameters in your tradespace, highlighting how point values in one parameter relate to point values in another. It visualizes and edits range annotations, which can create objectives and subsets.

The Compare Tool is access by clicking the Compare icon around the tradespace orbit (Figure 35) or by selecting Parallel Coordinates in the Combine tool.

Figure 35. Compare Tool tradespace orbit.

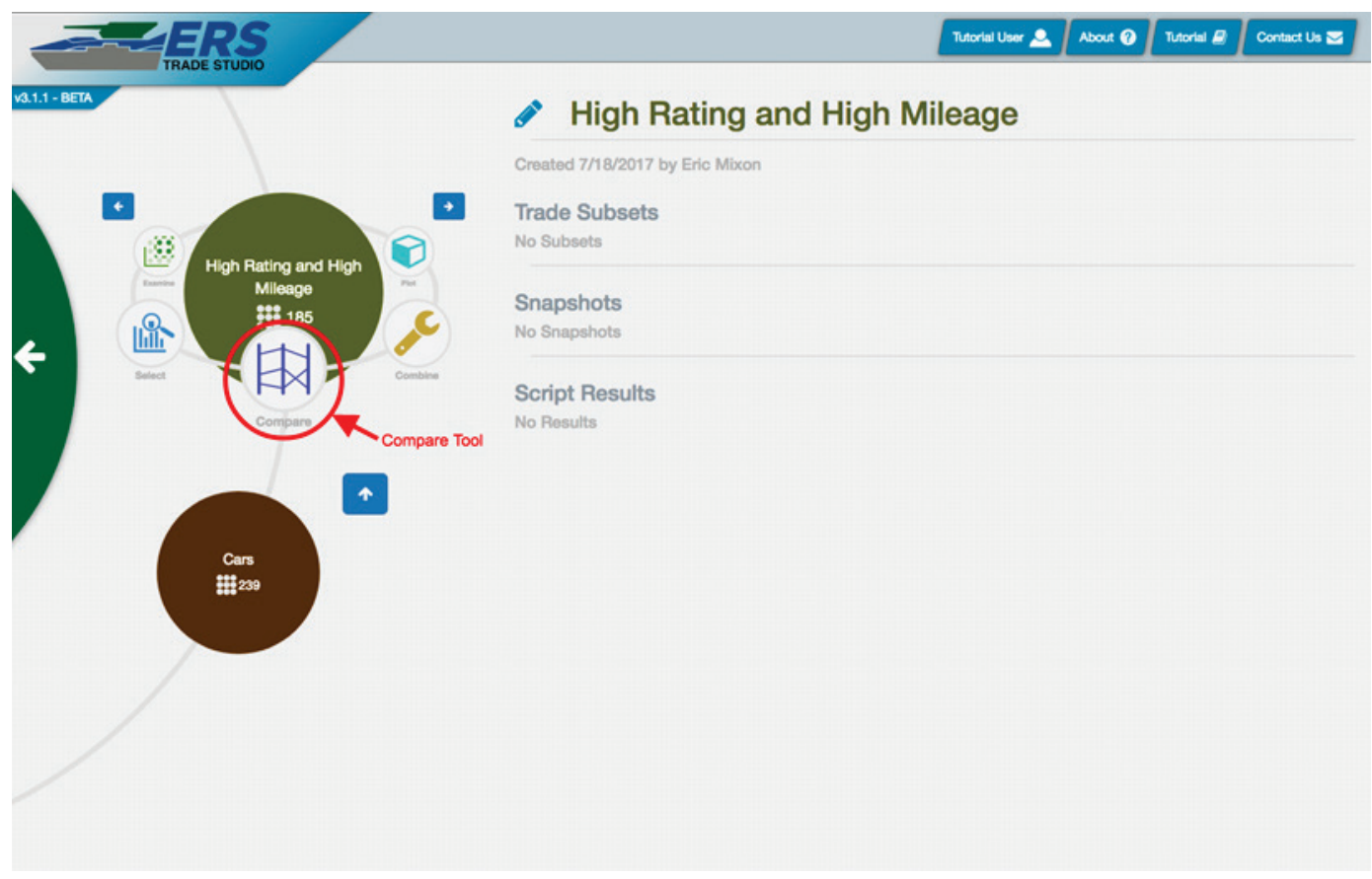

The Compare Tool creates range annotations and visualizes them across different parameters. Once a user creates a range annotation, the points within the range are highlighted across all selected parameters. If there are ranges across multiple parameters, only those points that fall in both parameters' ranges will be highlighted.

Note that because this view can visualize millions of points, the highlighted regions are binned, with increased opacity signifying more of that bin's points within the selection. 
Figure 36. Compare Tool selection view.

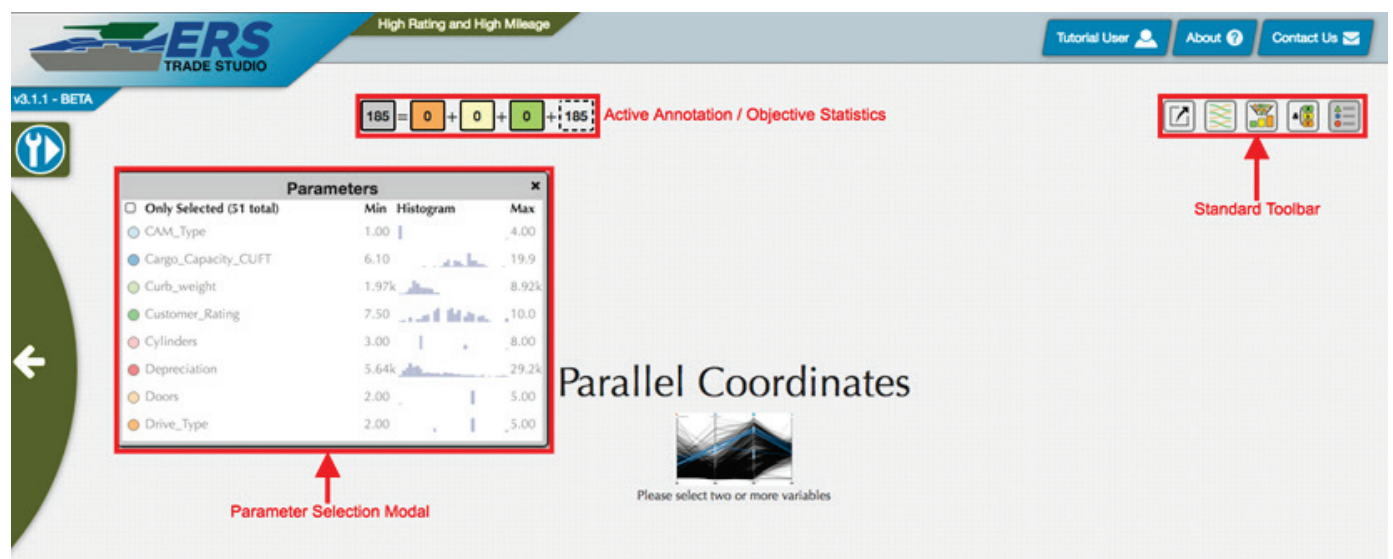

The initial view of the Compare Tool has the following features (Figure 36):

- Active Annotation - This shows the active annotation, as covered in the Annotations section.

- Standard Toolbar - This allows access to different tools in windows. The five tools from left to right are Export Package, Sankey Diagram, Objective Function Builder, Annotation Editor, and Parameter Selector.

- Parameter Selector - This selects and deselects parameters, as covered in the Parameters section. If there are not at least two parameters selected when arriving to the tool, this view will be open. Selecting two or more will activate the main view.

Once at least two points are selected, the tool will show the parameters as vertical lines with translucent lines connecting parts of the lines (Figure 37). The lines represent points that coincide in the bins connected by the line, and the opacity represents how many points are between those two bins. 
Figure 37. Compare Tool parameters selected.

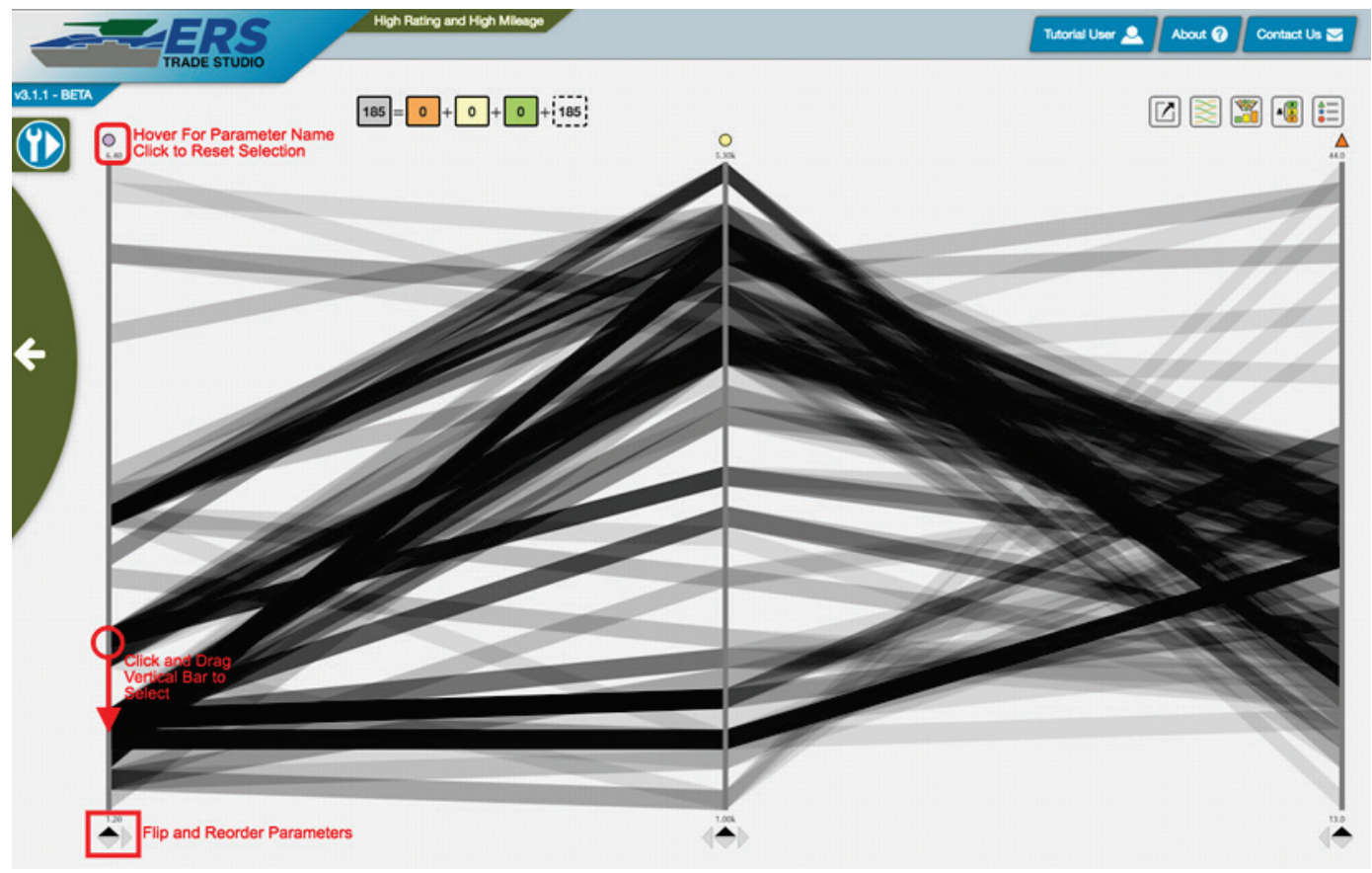

There are various controls for the visualization and annotations (Figure 37):

- Clicking and dragging the mouse along one of the parameter lines creates an annotation on that section. Multiple selections can be selected on a single parameter, allowing for values in both sections, and multiple parameters can have selections, requiring points that fall in parameter's selections.

- Any selection on a parameter can be removed by clicking the parameter's symbol on top of the line.

- The parameters can be flipped, so that low values are now on the top, to better visualize correlation between two parameters.

- The parameters can also be rearranged to visualize correlations between different parameters.

The ranges can be adjusted by placing the mouse along the edge of a selection (Figure 38). 
Figure 38. Compare Tool adjust annotation.

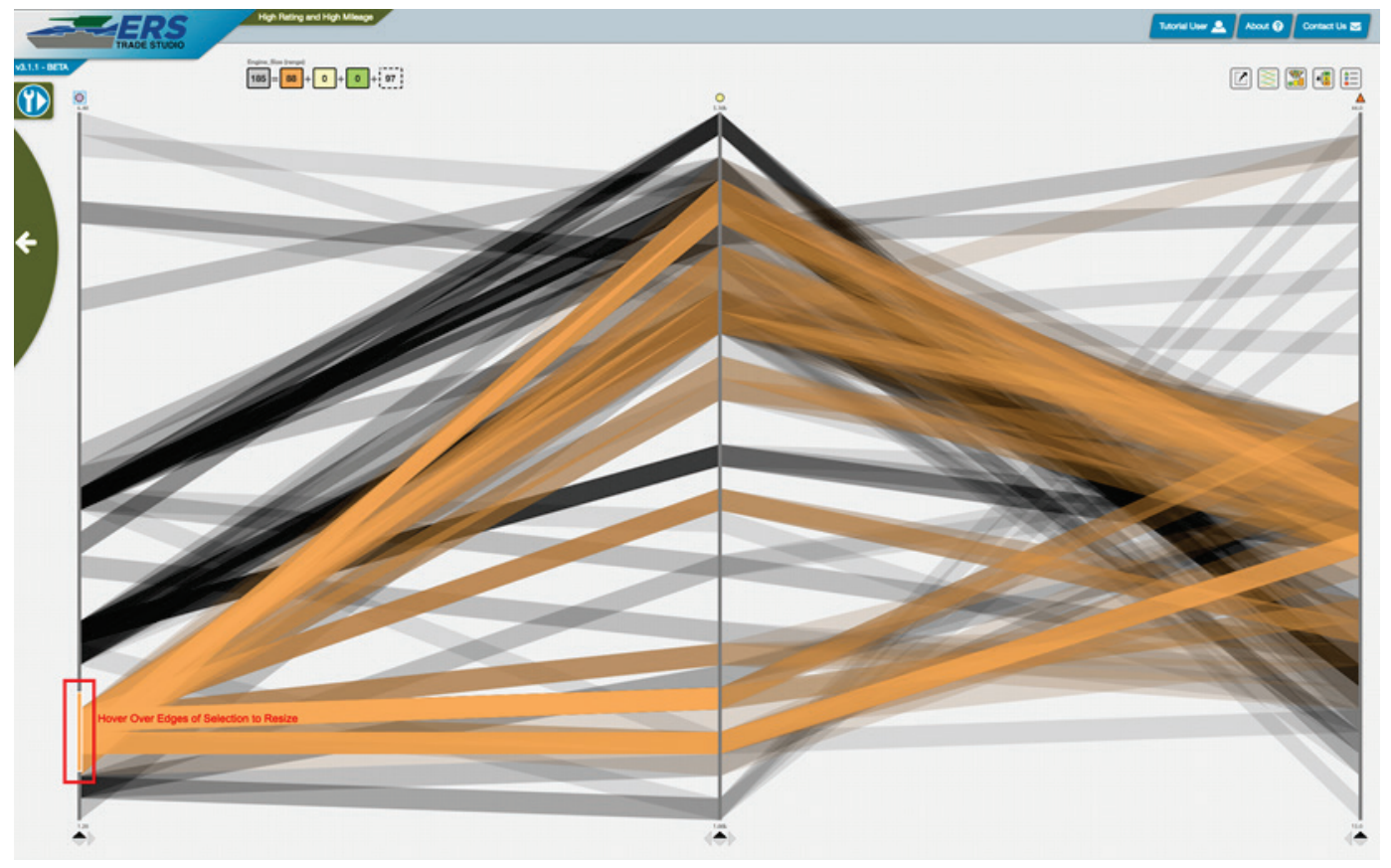




\section{Analyze Tool}

The Analyze Tool visualizes correlations between parameters. More accurately, it visualizes the mutual information of the parameters, which is the amount of information one can mathematically determine about one value using the other. This visualization hints toward which parameters are related and which bins of one parameter relate to other parameter's bins.

Mutual information is defined as the reduction in uncertainty due to another random variable. Entropy is how much uncertainty is in a parameter (also known as self-information). Pointwise mutual information is the mutual information of a single or a subset of events. These three measures point to important connections between parameters, both to show unexpected connections, and/or redundant parameters.

The Analyze tool is access by clicking the Analyze icon around the tradespace orbit (Figure 39), or by selecting Mutual Information in the Combine tool.

Figure 39. Analyze Tool tradespace orbit.

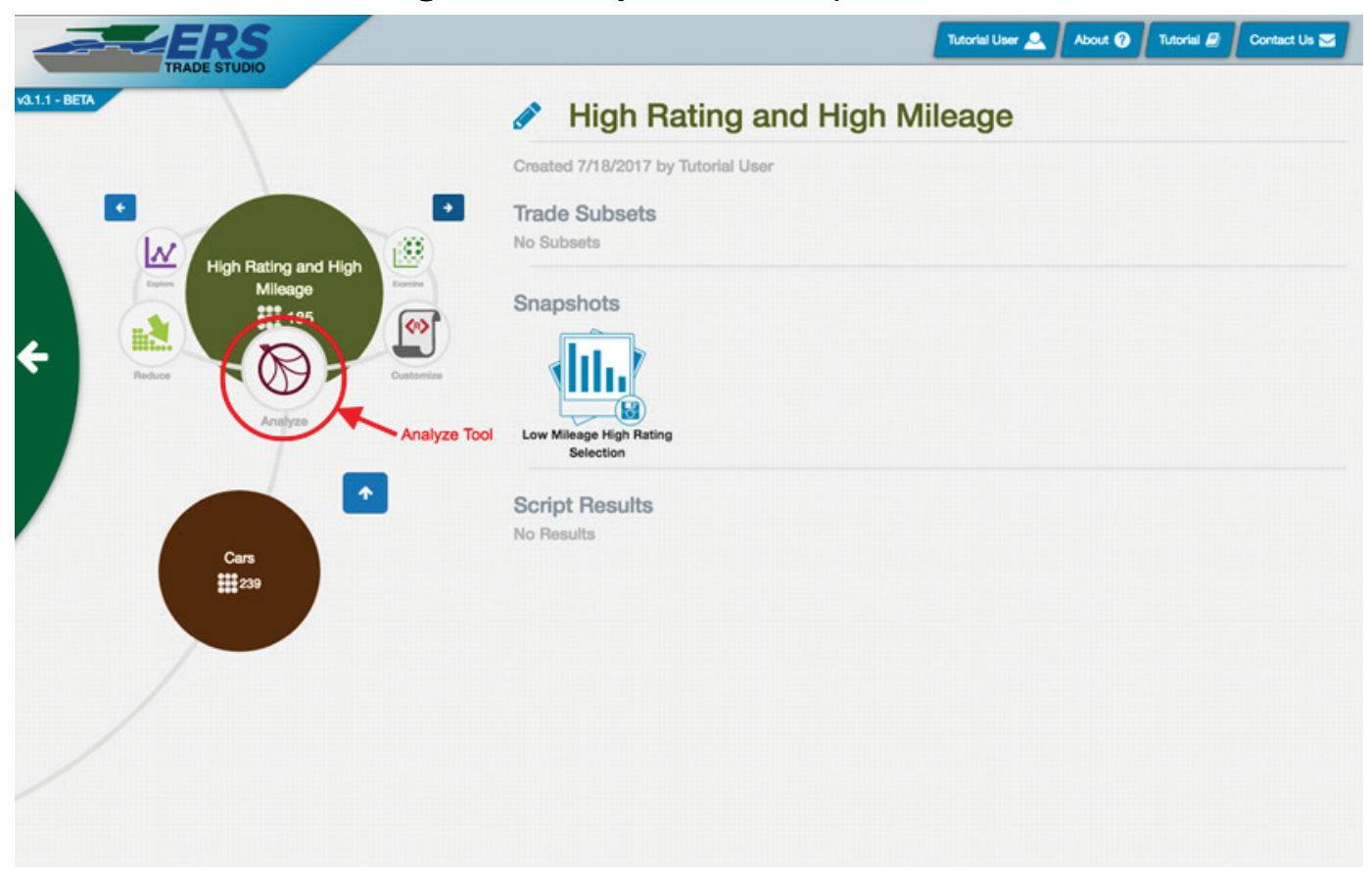


The initial view of the Compare is shown in Figure 40. The Standard Toolbar allows access to five different tools in windows: Export Package, Sankey Diagram, Objective Function Builder, Annotation Editor, and Parameter Selector. If at least two parameters are selected when arriving to the tool, the Parameter Selector will be open. Selecting two or more parameters activates the main view.

Figure 40. Initial view of the Compare window.

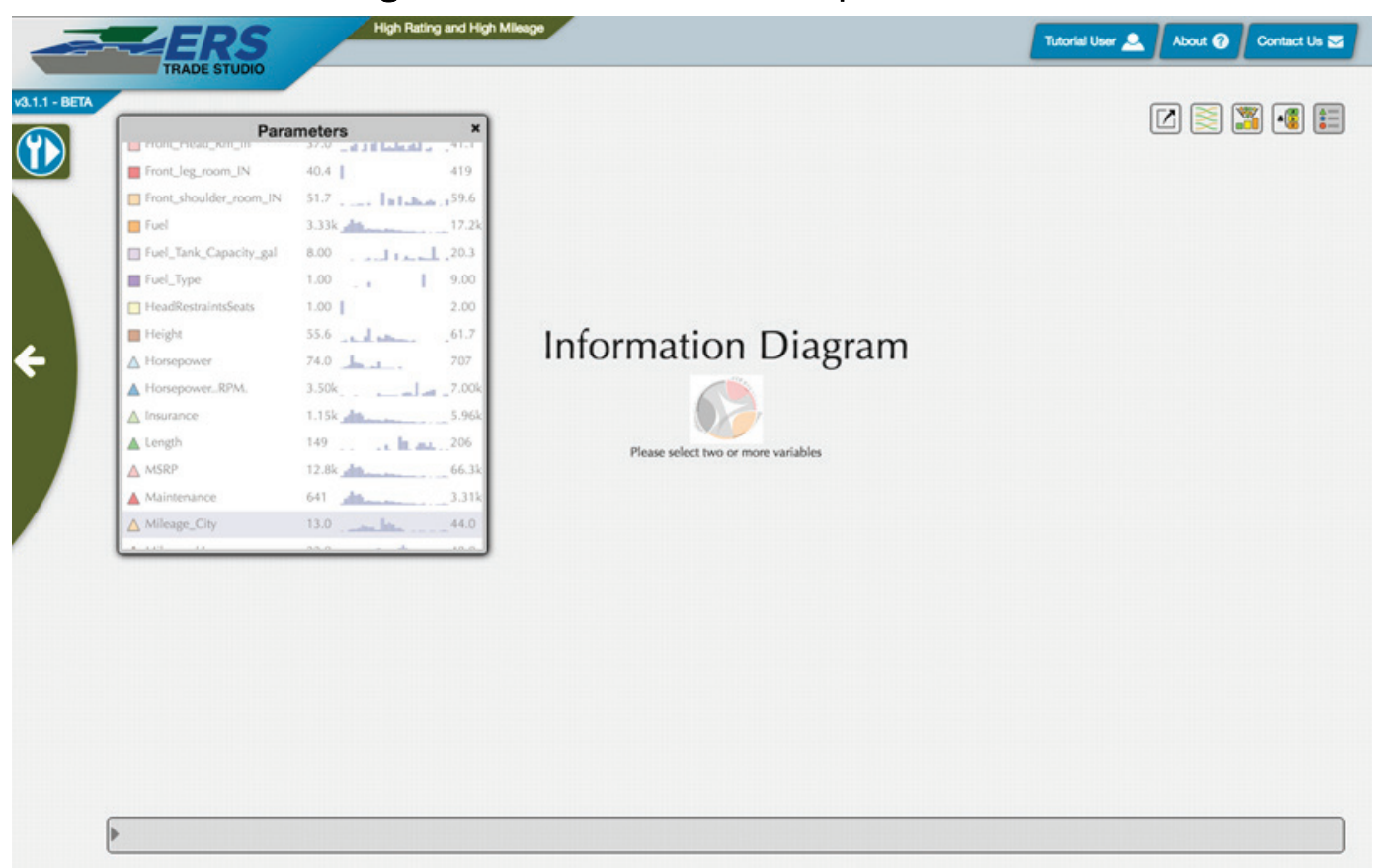

Once the parameters are selected, a circular chord chart appears representing the following metrics:

- Histograms - Around each parameter's arc, a histogram shows the parameter's distribution.

- Mutual Information - The thickness of the chords connecting parameters represents the mutual information between the two parameters.

- Entropy - The size of the hump next to the chords represents a parameter's entropy.

The visualization has the following functionality (Figure 41):

- Clicking a parameter's arc shows only that parameter's chords.

- Specific information will appear at the bottom when hovering over parts of the visualization. 
Figure 41. No parameters selected.

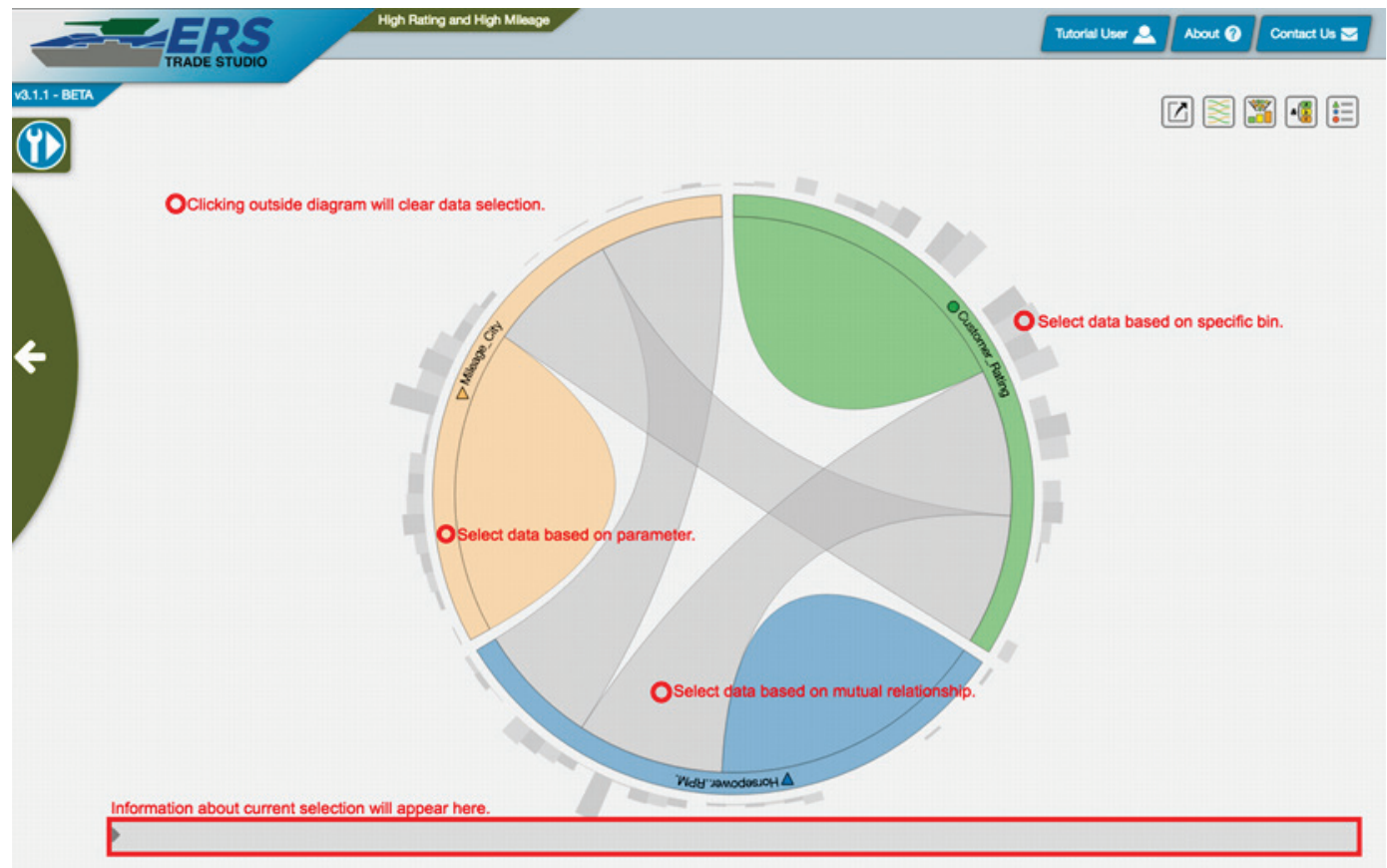

Clicking a chord between two parameters shows the pointwise mutual information between the bins (Figure 42). Only the bins that have a high amount have chords between them are shown to the user.

Figure 42. Two parameters selected.

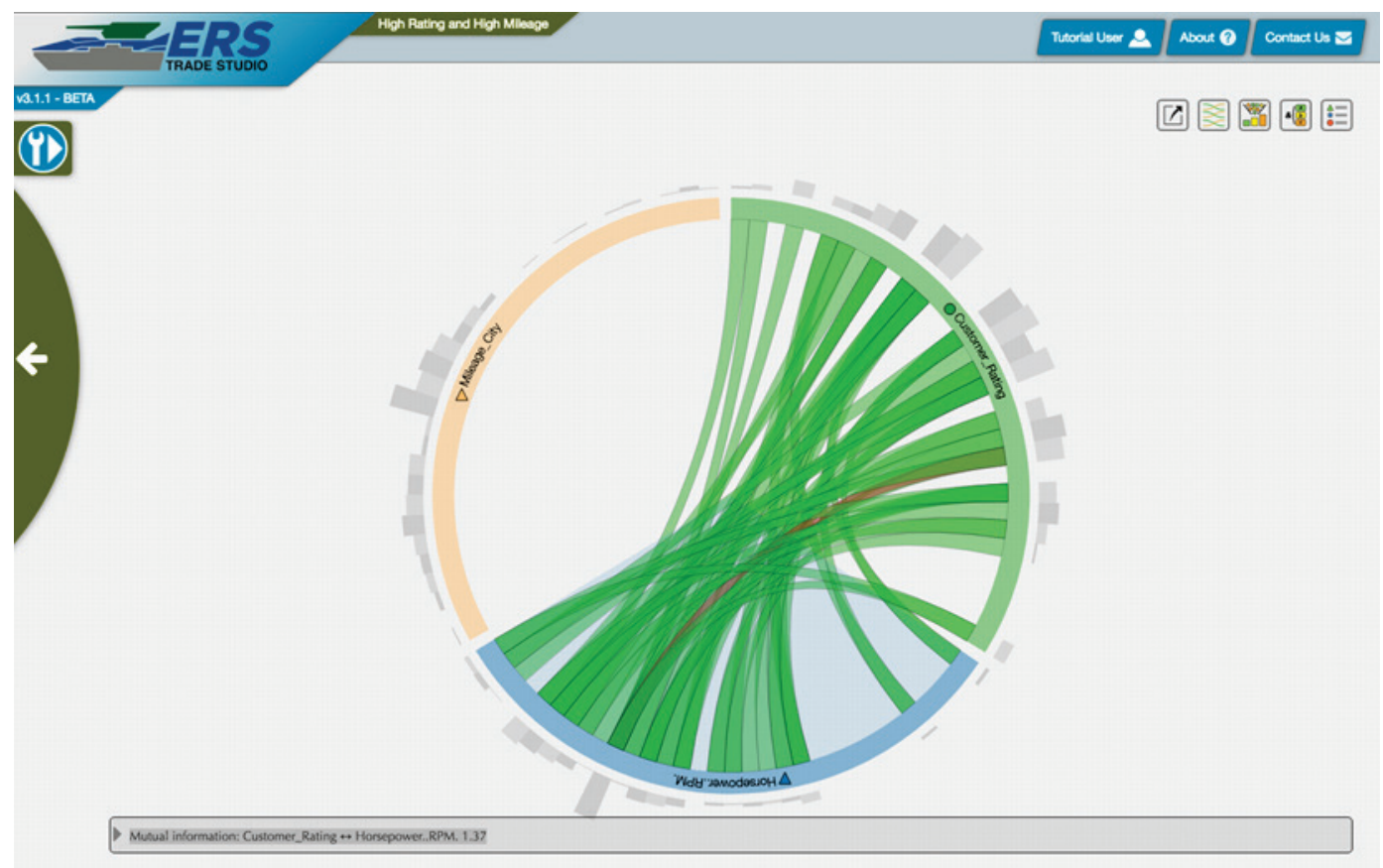


Clicking a specific bin shows chords to all other bins with high pointwise mutual information (Figure 43).

Figure 43. Single bin selected.

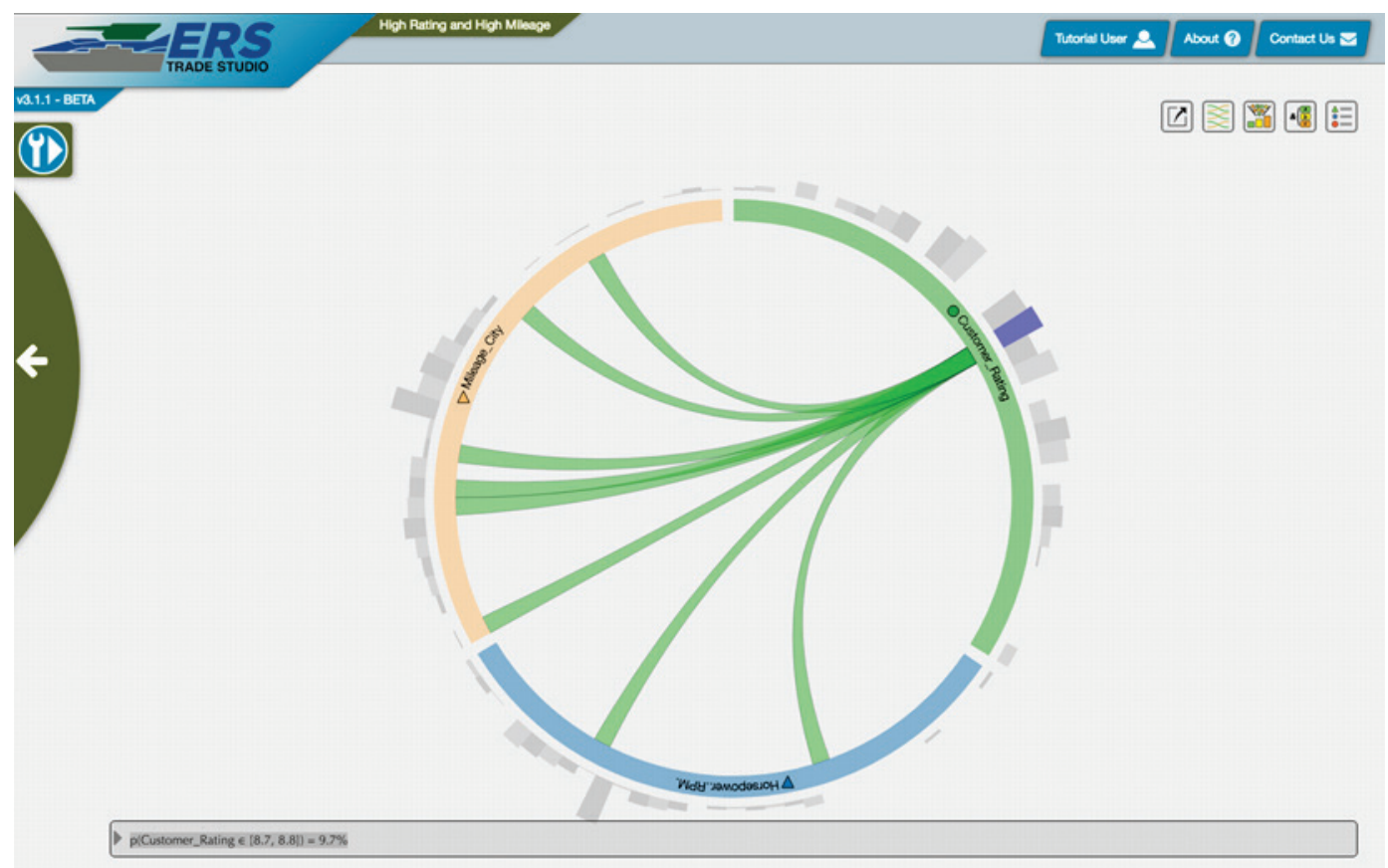




\section{Reduce Tool}

The Reduce Tool allows a user to see more information about a particular parameter in the tradespace using histograms (Figure 44). There is a histogram for each parameter in the tradespace. The following is a list of available features within the tool.

- Filters - Allows the user to view the parameters based on the currently selected parameters, scores, and rows.

- Accumulative Score - Allows the user to view the score based on the dividends of the annotation view.

- Select/Unselect - The user can narrow the parameter set by selecting or deselecting the parameters.

- Save Annotation - Allows the user to save their annotation.

- Activate Annotation - Activates annotation editing.

- Expand Parameter View - Enables full-screen for the currently selected parameter.

- Functionality Tools - From left to right, respectively.

- Export Package - Refer to the Export section.

- Sankey Diagram - Refer to the Analyze Tool section.

- Objective Function - Refer to the Objective section.

- Annotations - Refer to the Annotations section.

- Parameters - Refer to the Parameters section. 
Figure 44. Reduce Tool home page.

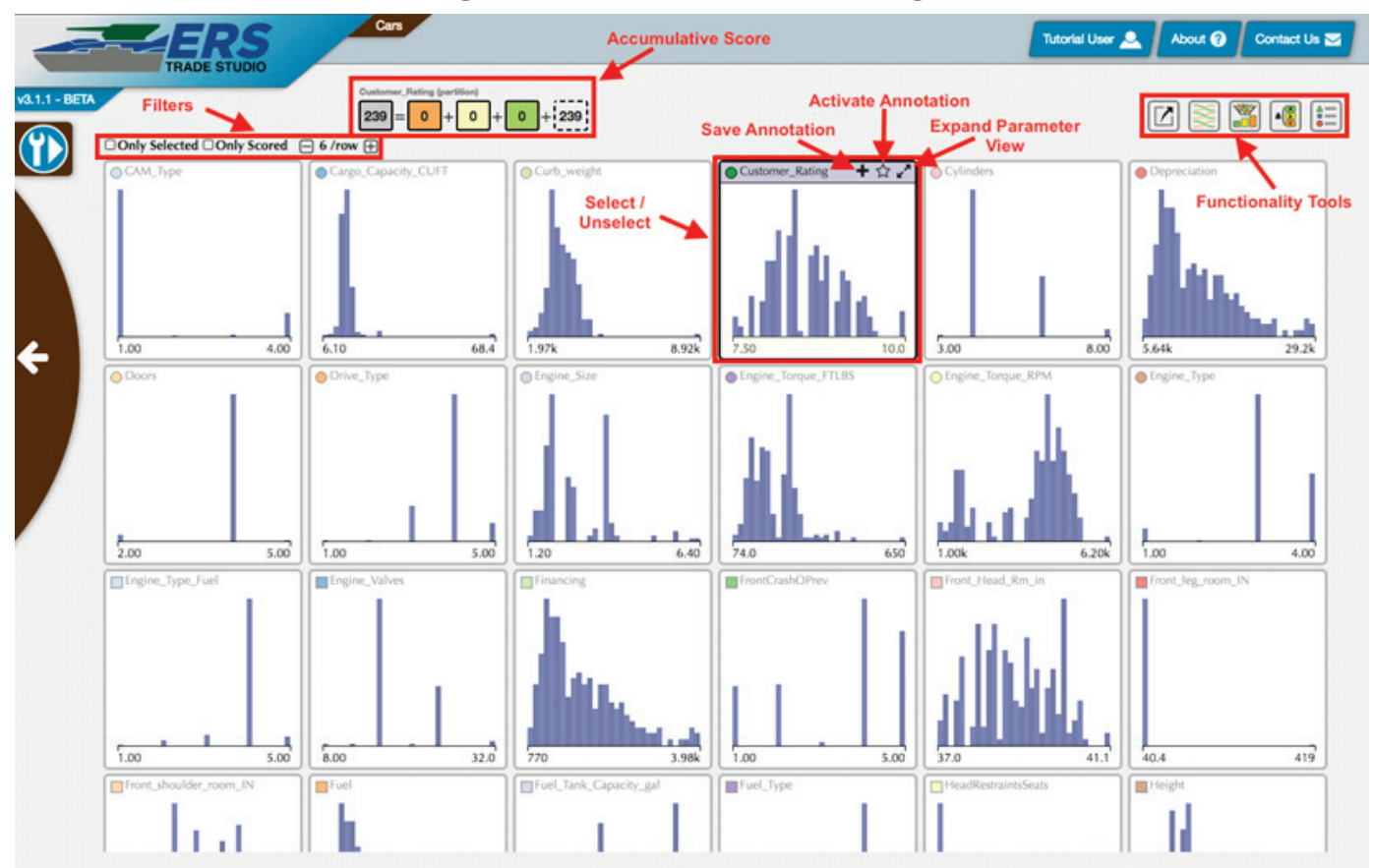




\section{Plot Tool}

The Plot Tool allows a user to see a 3D scatterplot of the currently selected tradespace. The visualization is computed on the server and sent to the website as images. This allows more computationally intensive visualizations than are possible within the browser.

The Plot Tool is accessed by clicking the Plot icon around the tradespace orbit (Figure 45) or by selecting $3 D$ Scatterplot in the Combine tool.

Figure 45. Plot Tool Orbit.

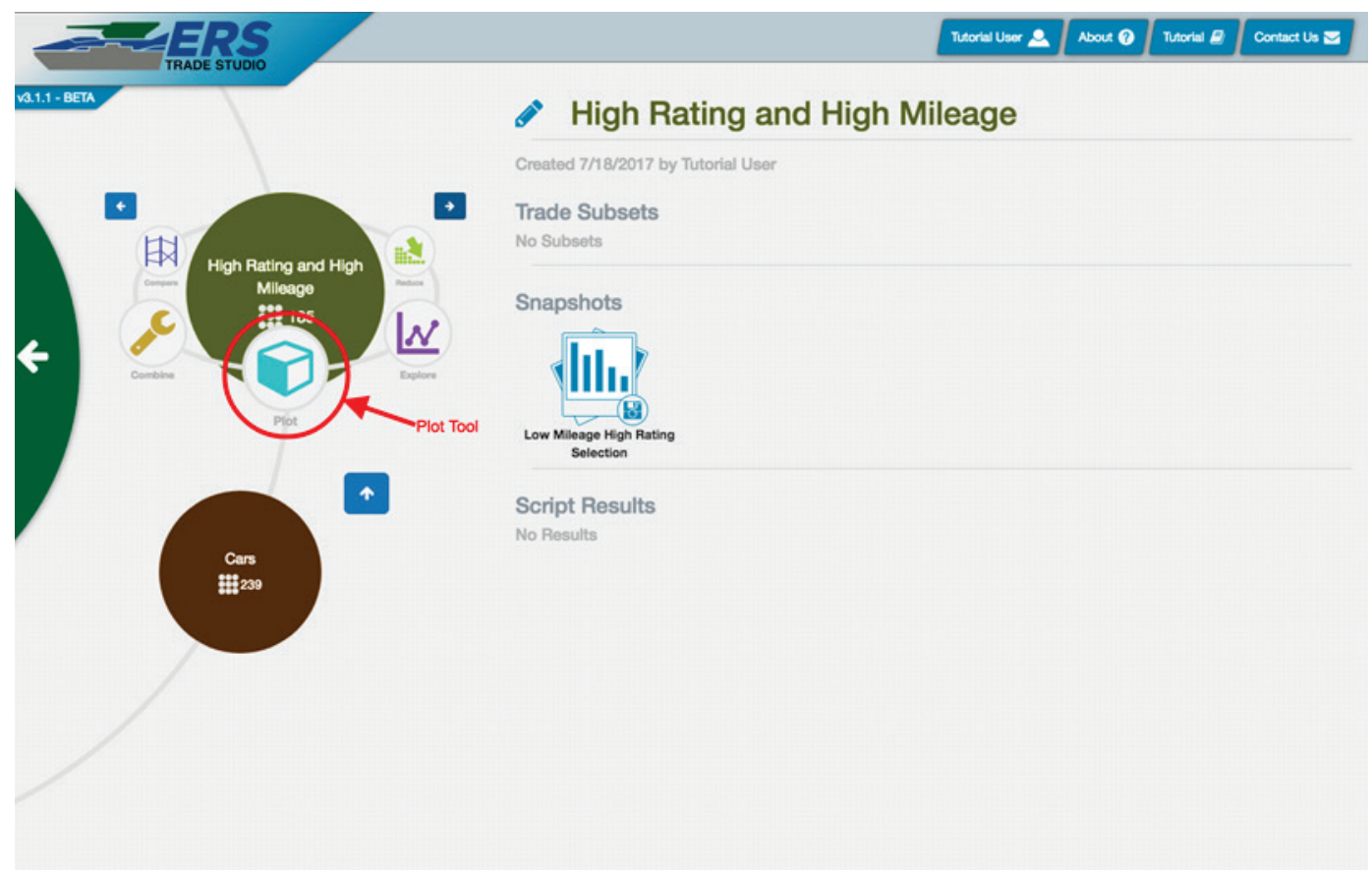

When a user first arrives to the $3 \mathrm{D}$ Scatterplot tool page, there will be customizable $3 \mathrm{D}$ representation of data (Figure 46). 
Figure 46. Plot tool - home view.

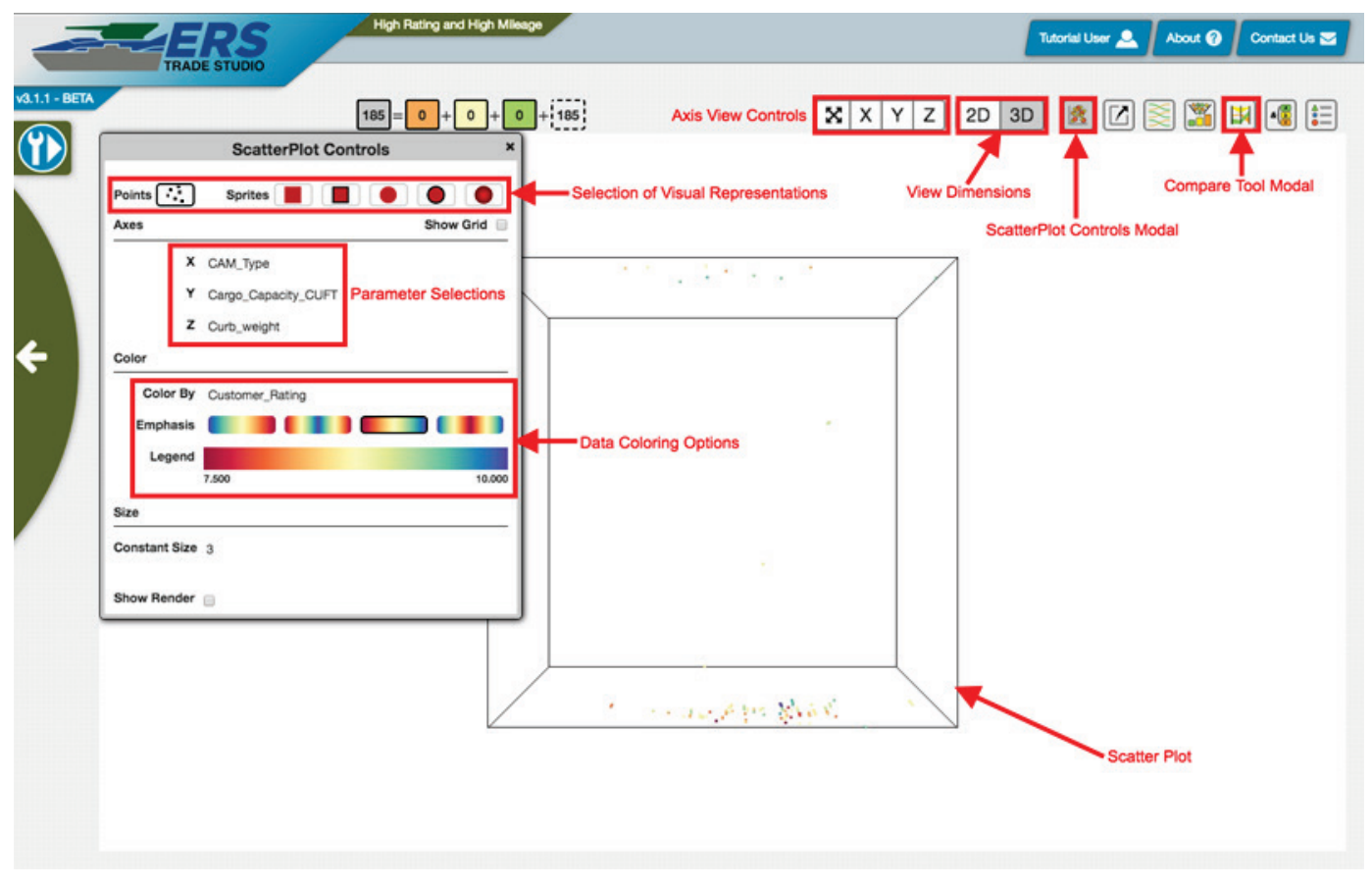

A user can move around the visualization in various ways. Clicking and dragging rotates the graph. Scrolling the mouse wheel, or Ctrl + click and drag, zooms in or out. Shift + click and drag rotates the graph around the Z-axis (i.e., if you were rotating a picture on a wall). In the top of the screen are the axis view controls, this allows the user to either select the $\mathrm{X}$, $\mathrm{Y}$, or $\mathrm{Z}$ axis to orient the view along the respective axis and center the view. Next to the axis controls is the option to switch between $2 \mathrm{D}$ and $3 \mathrm{D}$ views.

The Scatterplot Controls button in the top right opens the Scatterplot Controls window on the left side of the screen (Figure 46). The initial options are as follows:

- Point/Sprite type - These options allow for different ways for rendering the individual points. The Points option is less computationally intensive, this is good for visualizing millions of points. The sprites option, while more computationally intensive, are more visually distinct and offer more options to be discussed later.

- Show Grid - This option shows the axis grid lines. Turning this option on can reduce the performance while moving the graph.

- Parameter Axes Selection - Users can decide which parameters are which axes.

- Show Render Stats - This reveals rendering statistics and can help diagnose what is making the visualization poor. Common causes could 
be slow network connection, overtaxed server, and poor handling by the client.

If the Sprite option is selected, additional options are available in the Scatterplot Controls window (Figure 47). A point's size and opacity can scale with a parameter or an annotation. How they scale can be customized, such as changing whether large points have high points or lower or how large the points can get.

Figure 47. Plot Tool - additional options.

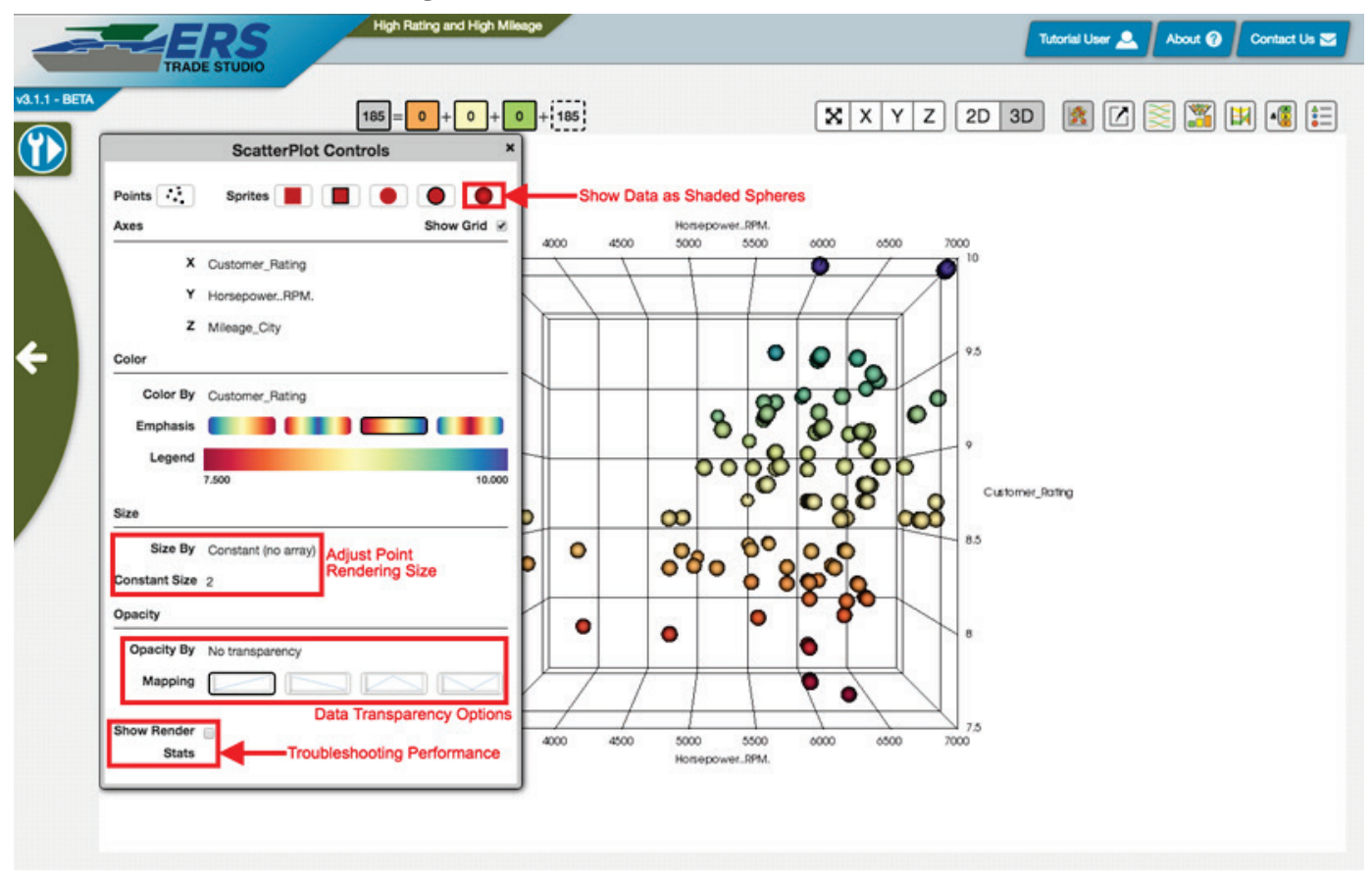

An additional functionality is the Compare Tool popup. Clicking the Compare icon in the top right opens this popup (Figure 48). This popup allows an easy way to change the active annotation. Complete coverage of how to use the Compare Tool is in the Compare Tool section. 
Figure 48. Plot Tool - Compare Tool annotations.

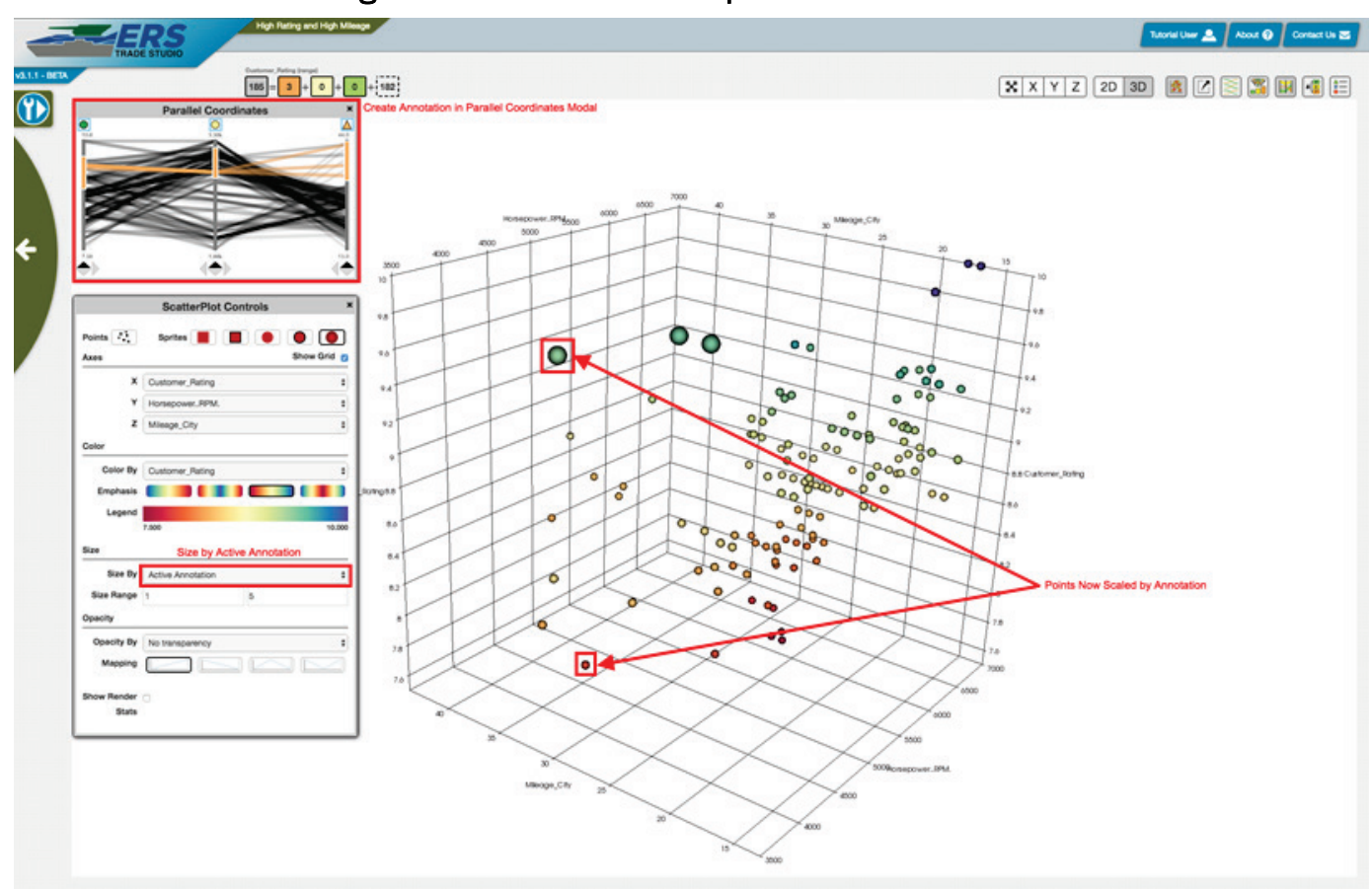

A user can change either Size by or Opacity by to the active annotation, changing the respective selection by the current annotation. The Compare pop-up can change the annotation, allowing responsive visualization of the annotation without changing the view. The annotation editor (in the top right) can change the annotations further and is covered in the Annotations section. 


\section{Combine Tool}

The Combine Tool merges multiple tools in one location, so that the correlations of data can be simpler to visualize (Figure 49). This tool includes the mutual information diagram, histograms, parallel coordinates, Plotly charts, 3D scatter plot, and field selector. The following additional information can be found at:

- Mutual Information - Refer to the Analyze Tool section.

- Histograms - Refer to the Reduce Tool section.

- Parallel Coordinates - Refer to the Compare Tool section.

- Plotly Charts - Refer to the Explore Tool section.

- $3 D$ Scatter Plot - Refer to the Plot Tool section.

- Field Selector - Refer to Parameters section.

- Workbench - Allows the user to change the chart types, parameters, and panel layout.

Figure 49. Combine Tool Home Page.

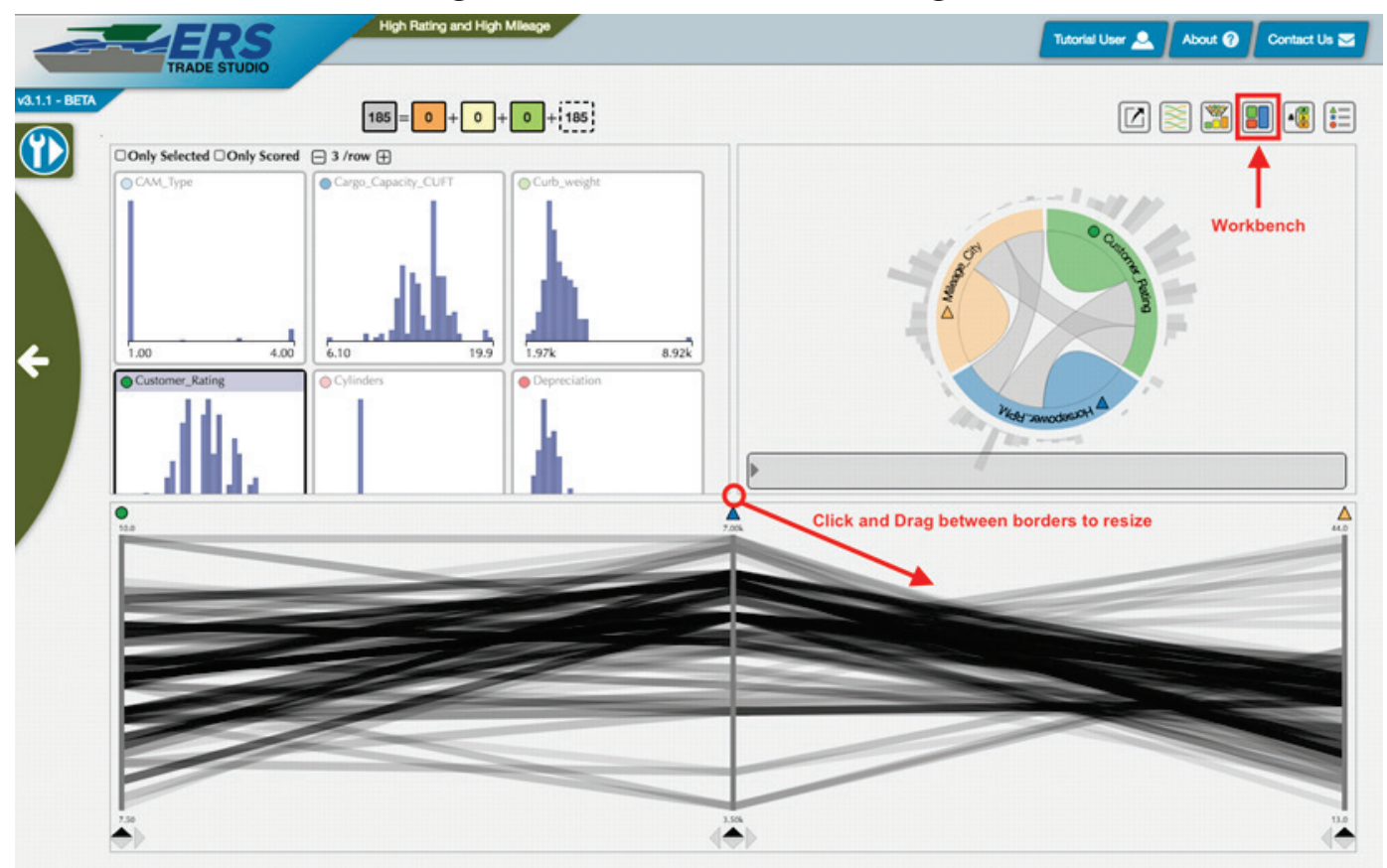

When a user first accesses the workbench, they will see various tools. Some tools, such as the mutual information diagram, can only be accessed once two or more parameters have been selected. 
In the workbench layout, the user can specify the panel layout by clicking on any of the provided icons. The user can specify which tool they want for each panel (Figure 50).

Figure 50. Workbench layout.

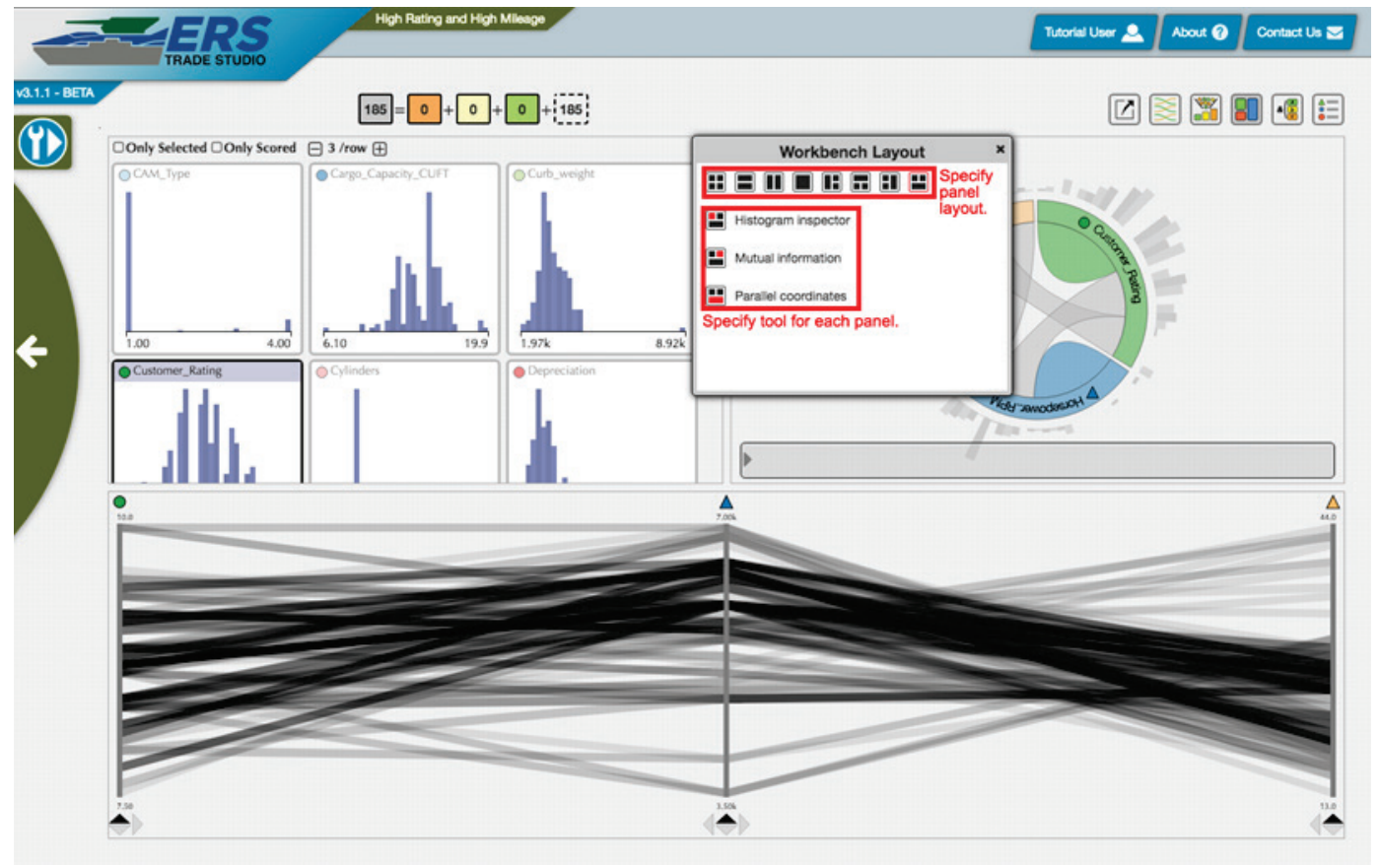

When the user selects information within one of the tools, the changes will be reflected in the other tools. 


\section{Examine Tool}

The Examine tool allows users to view scatter-plots and histograms related to their selected parameters, and to select individual points on those charts for later use.

A 2D histogram will exist for each parameter selected, and a scatterplot matrix will exist for every relationship that exists between two or more selected parameters. On these histograms, individual points can be selected. If there are no selected parameters, no charts will appear (Figure 51).

Figure 51. Examine tool no parameters selected.

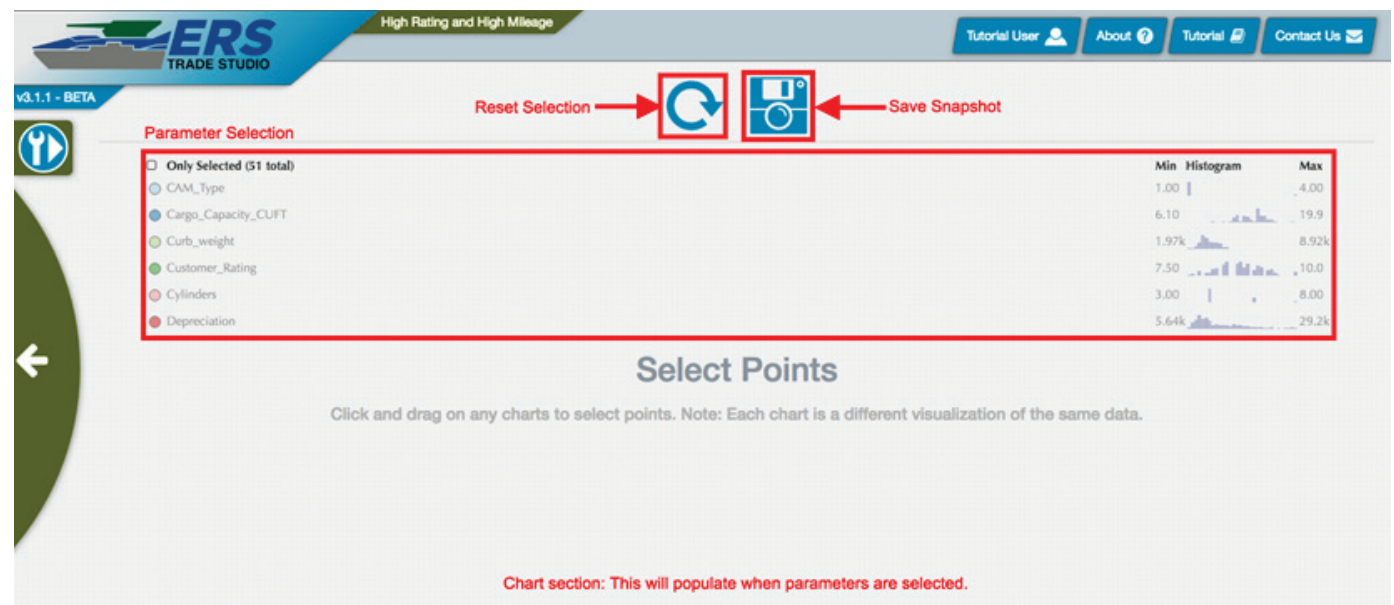

Below is a list of other available features on the webpage (Figure 52):

- Reset Selection - Deselects all points and parameters currently set, along with resetting the current analysis.

- Save Snapshot - The snapshot icon will save a snapshot of the currently selected points and parameters. A snapshot will allow a user to instantly return to the current view with the same points, parameters, and analysis.

- Parameter Selection - Allows the user to change the currently selected parameters. 
Figure 52. Examine tool parameters selected.

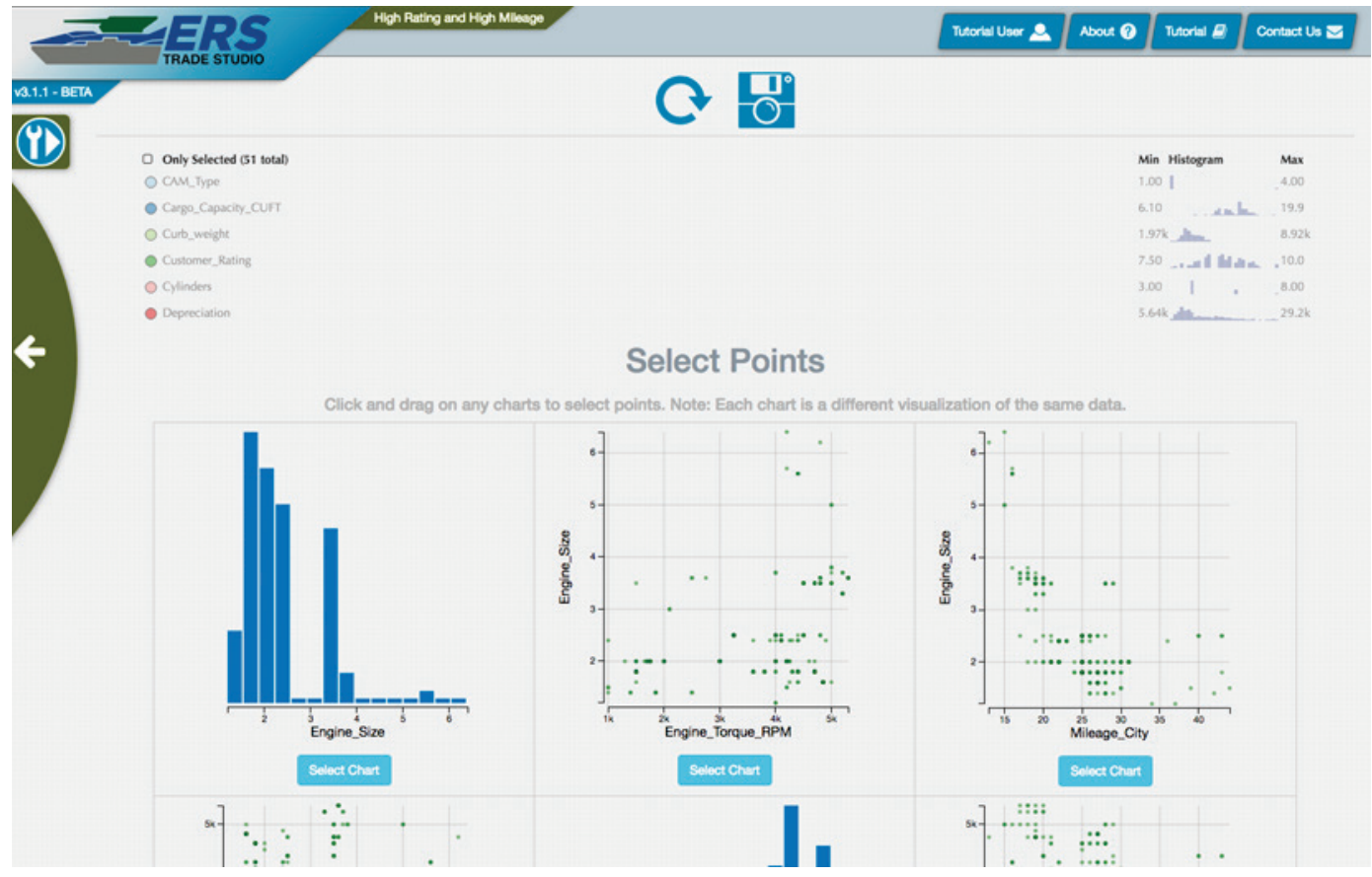

Once the parameters have been selected, the histograms and scatterplots related to those parameters will be shown. Each chart can be selected for a more detailed view by clicking Select Chart (Figure 53).

Figure 53. Select chart view.

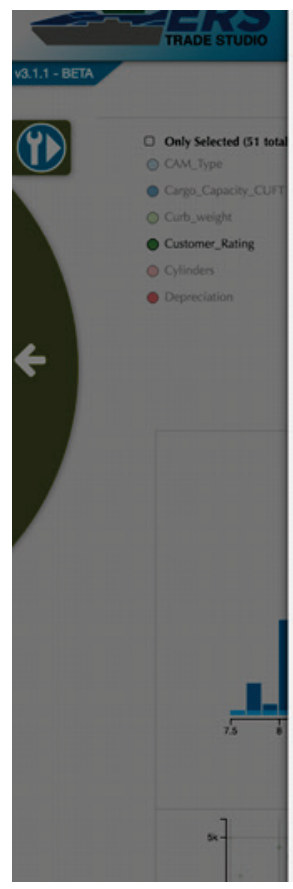

Customer_Rating vs. Mileage_City
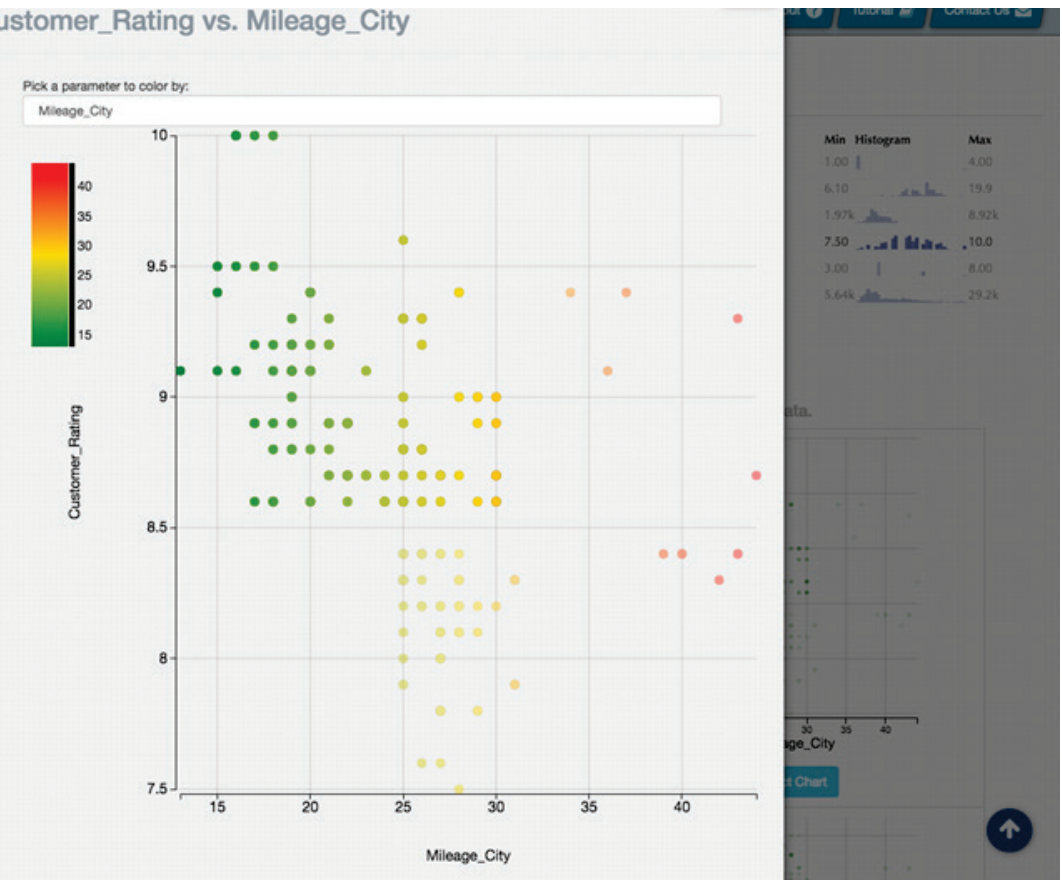
In this view, points can be individually selected and the chart can be colored by particular parameters. To exit the select chart view, click the $x$ in the top right corner of the select chart window to return to the examine tool.

Once the user has made their selections, they can save a snapshot for later viewing by clicking the Save Snapshot button.

At any time, a user can return to the tradespace tool orbit by hitting the arrow at the left border of the screen. 


\section{Select Tool}

The Select tool looks at individual points, allowing for user-defined data point selections, saving points, and comparisons using objectives (Figure 54). This requires data to be loaded into the browser, therefore, it cannot be used on vast data sets.

Figure 54. Select tool home page.

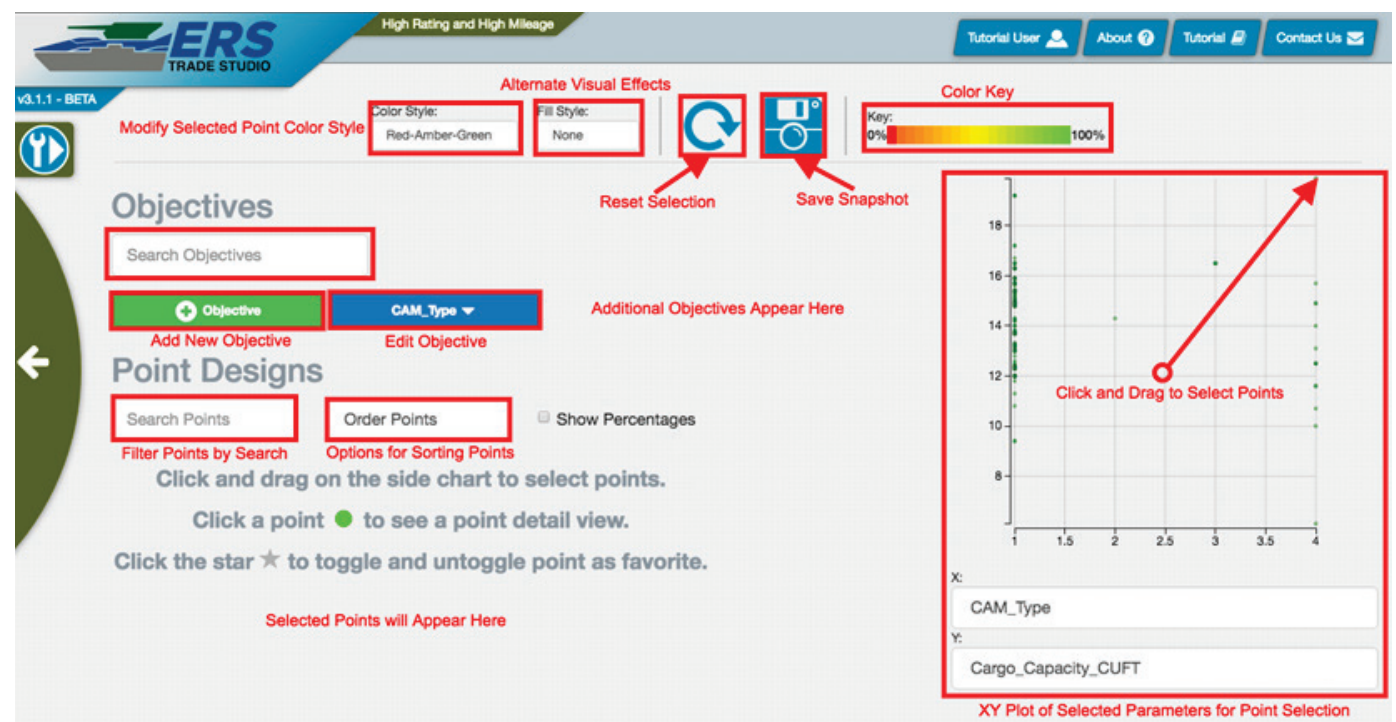

The following is a list of the available features:

- Color Style - Allows the user to select their preferred color style to accurately represent their selected data. The following three styles are available: Red-Green-Amber, Greyscale, and Black

- Fill Style - Allows the user to select their preferred fill style. This affects the way the points are displayed to the user. The fill levels depend on the chosen objectives. There are four available styles: None, Moons, Pie Charts, and Circles.

- Reset All - Deselects all points and parameters currently set, along with resetting the current analysis.

- Snapshot - The snapshot icon will save a snapshot of the currently selected points and parameters. A snapshot will allow a user to instantly return to the current view with the same points, parameters, and analysis. 
- Color Key - Shows the colors associated with the currently selected color style. The colors coordinate with its equivalent percentage rate. If a point met $0 \%$ of the objectives, it would be red on the Red-AmberGreen color style. If a point met $100 \%$ of the objectives, it would be green.

- Search Objectives - Allows the user to filter specific objectives. This is useful if the user has a large number of objectives.

- Add Objectives - Allows the user to add a new objective by clicking the green button labeled objective.

- Edit Objective - Allows the user to edit the selected objective. The available features are shown in Figure 55 .

Figure 55. Select tool Edit Objective.

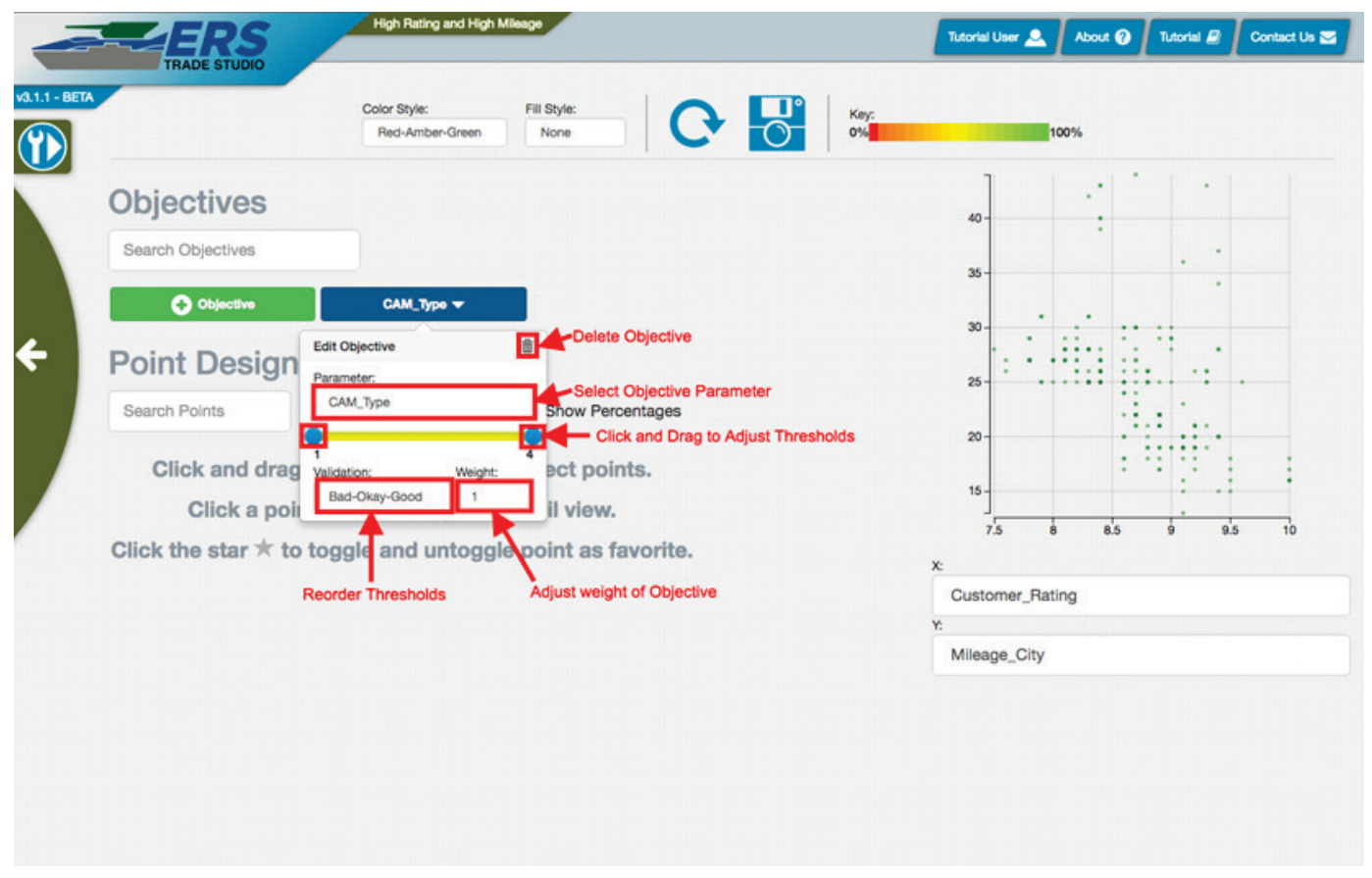

- Delete Objective - Allows the user to delete the objective by clicking the trashcan icon.

- Objective Parameter - Allows the user to change the currently selected objective. After clicking in the box, a drop down list of the available parameters will become visible.

- Adjust Thresholds - Allows the user to adjust the thresholds of the given objective. There are two blue buttons that can be adjusted on either side. This gives the user the ability to alter the range of acceptability. Figure 56 shows the Mileage_City objective threshold being adjusted. 
Figure 56. Select Tool Adjusting Thresholds

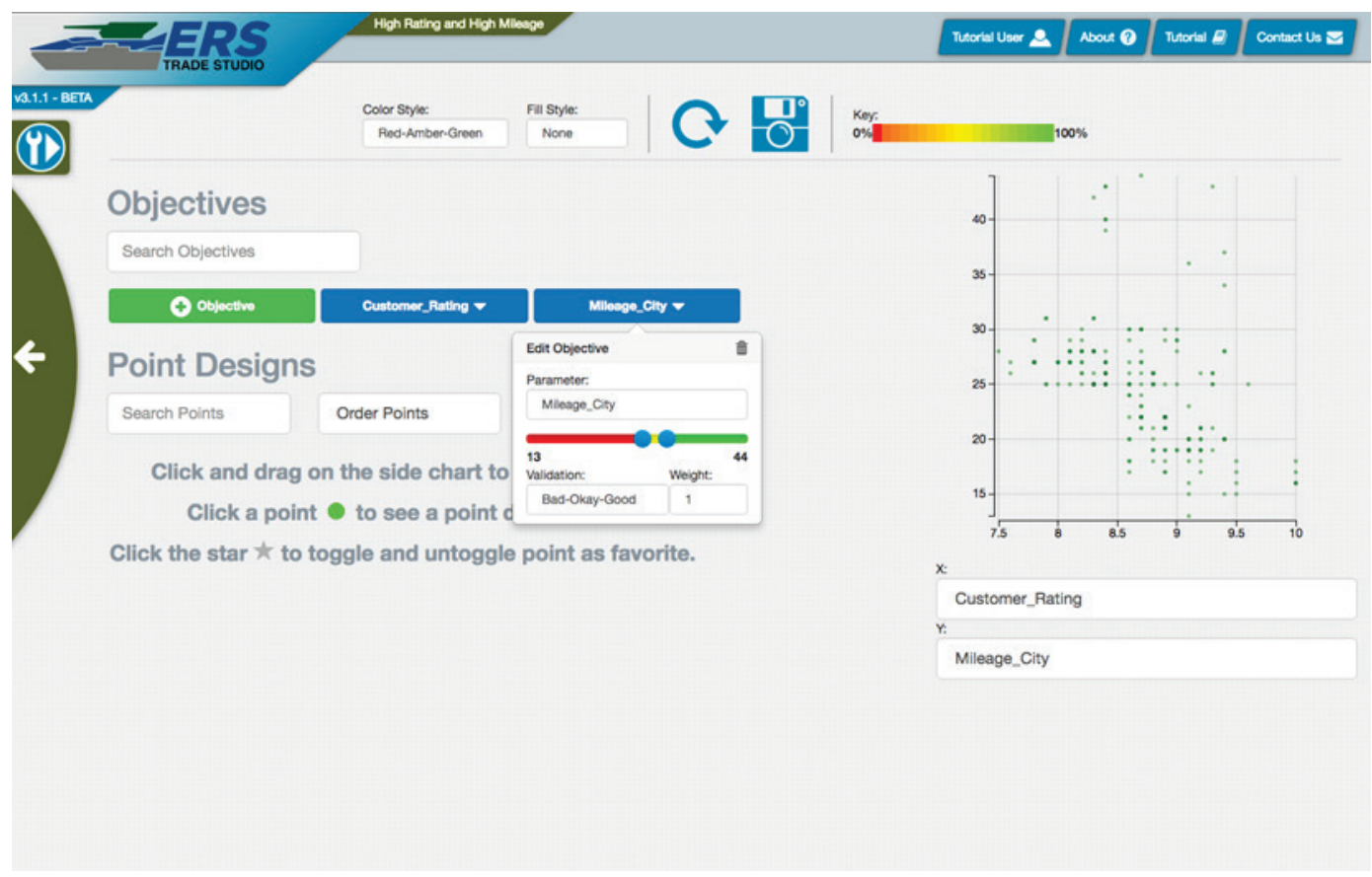

- Validation - Allows the user to reorder the validation threshold. Changing these values will switch the order in which the values are interpreted. The following four options are available: Bad-Okay-Good, Good-Okay-Bad, Bad-Good-Bad, and Okay-Good-Okay.

- Weight - Allows the user to adjust the impact of the given objective. There is a range from one to five, along with the option to ignore the impact. When a higher weight is applied to the objective, the more impact it has on the overall objective score. One would be a low impact, while five would be a high impact. Ignore means this objective does not influence the decision within the objective.

- Search Points - Allows the user to search for specific data points. This is useful if the user has selected a large amount of data points.

- Order Points - Allows the user to order the points by parameters, scores, or favorites (Figure 57). 
Figure 57. Select Tool Order Points.

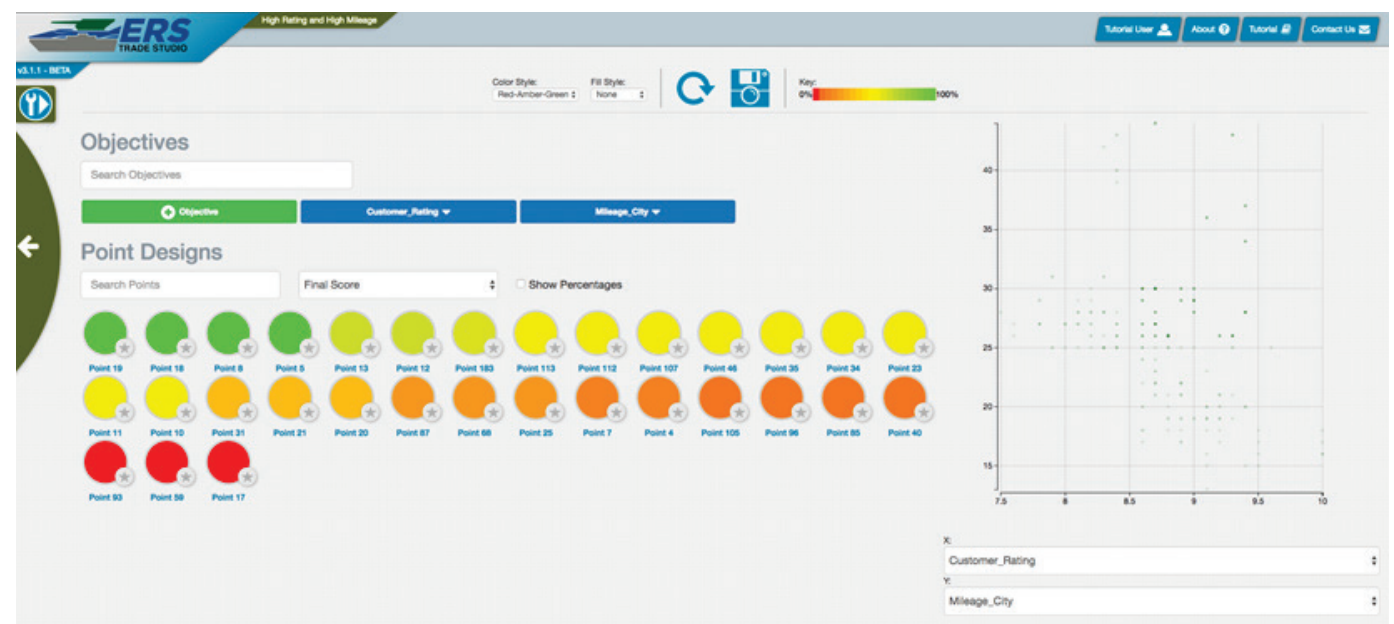

- Show Percentages - Shows the percentage of how the current point is meeting the selected objective.

- Favorite - The user can click the star icon on a point to set it as a favorite point (Figure 58).

Figure 58. Select Tool Setting Favorites.

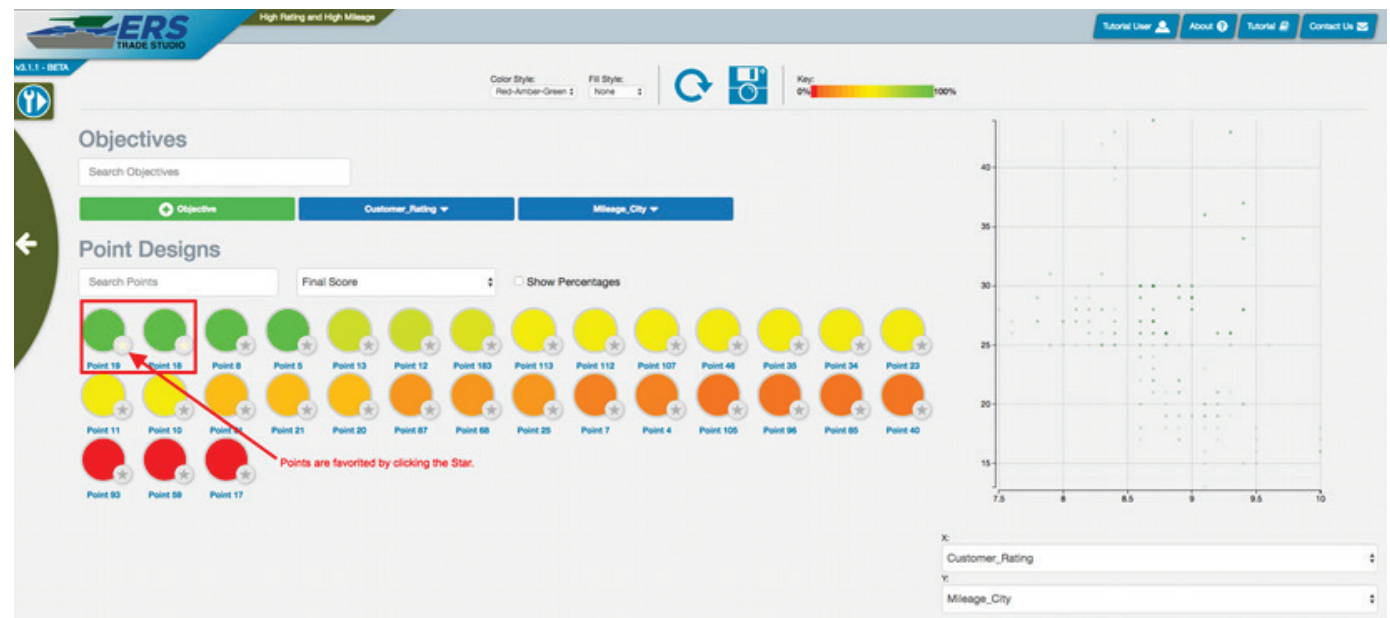


- Selecting Data Points - Before any of the functionality can be used, the user must first select a set of data points (Figure 59). To select a set of data points, the user must click and drag their mouse over the area of interest. The selected points will appear below the point design section.

Figure 59. Select Tool Choosing Data Points.

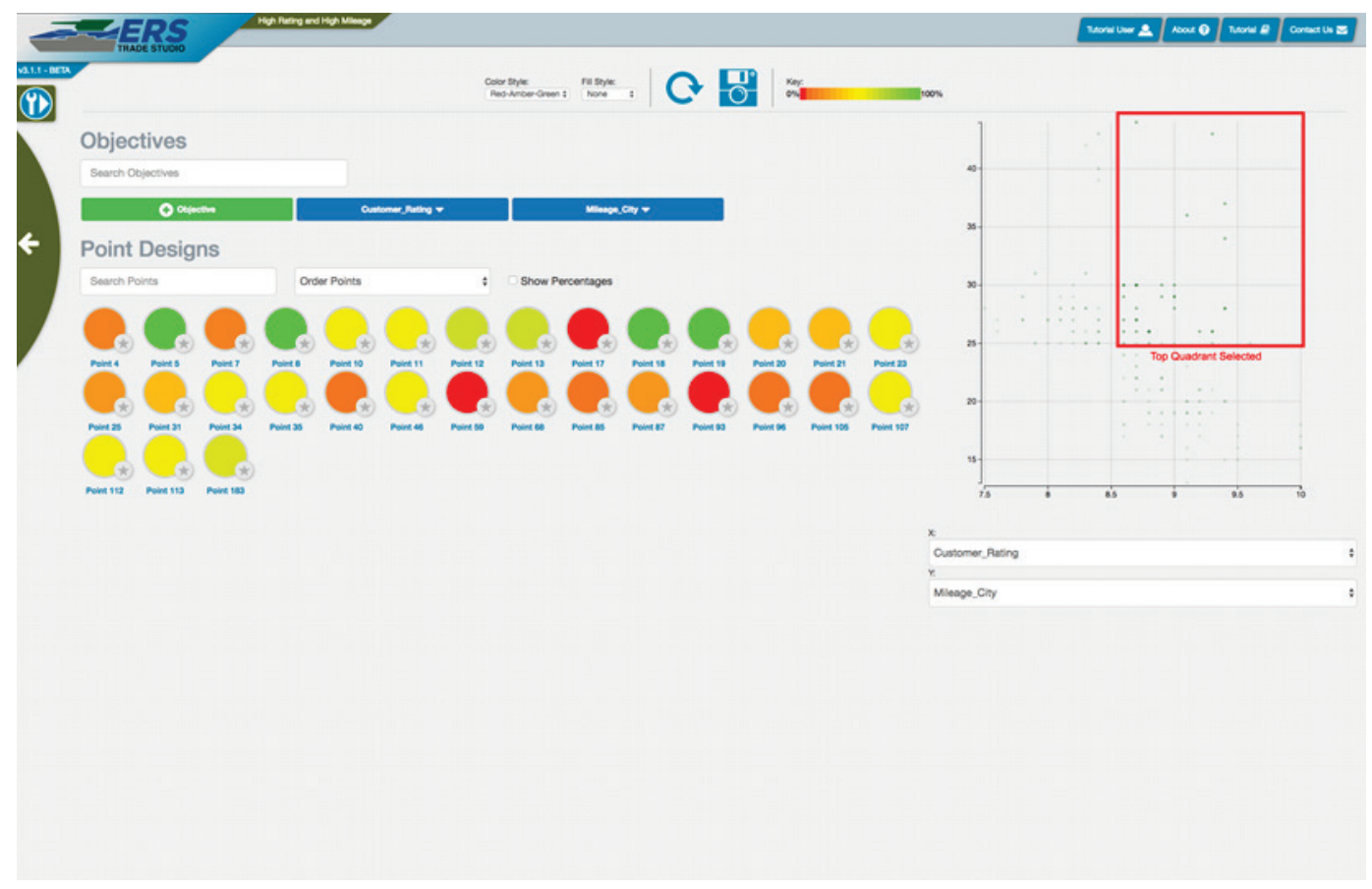

- Modify X-Axis - Allows the user to select the X coordinate from a list of available parameters.

- Modify Y-Axis - Allows the user to select the Y coordinate from a list of available parameters.

The point comparison allows the user to compare two data points by displaying the current objectives and parameters of the specified points. This page will only be visible once a point is selected (Figure 60). 
Figure 60. Select Tool Points Comparison Page

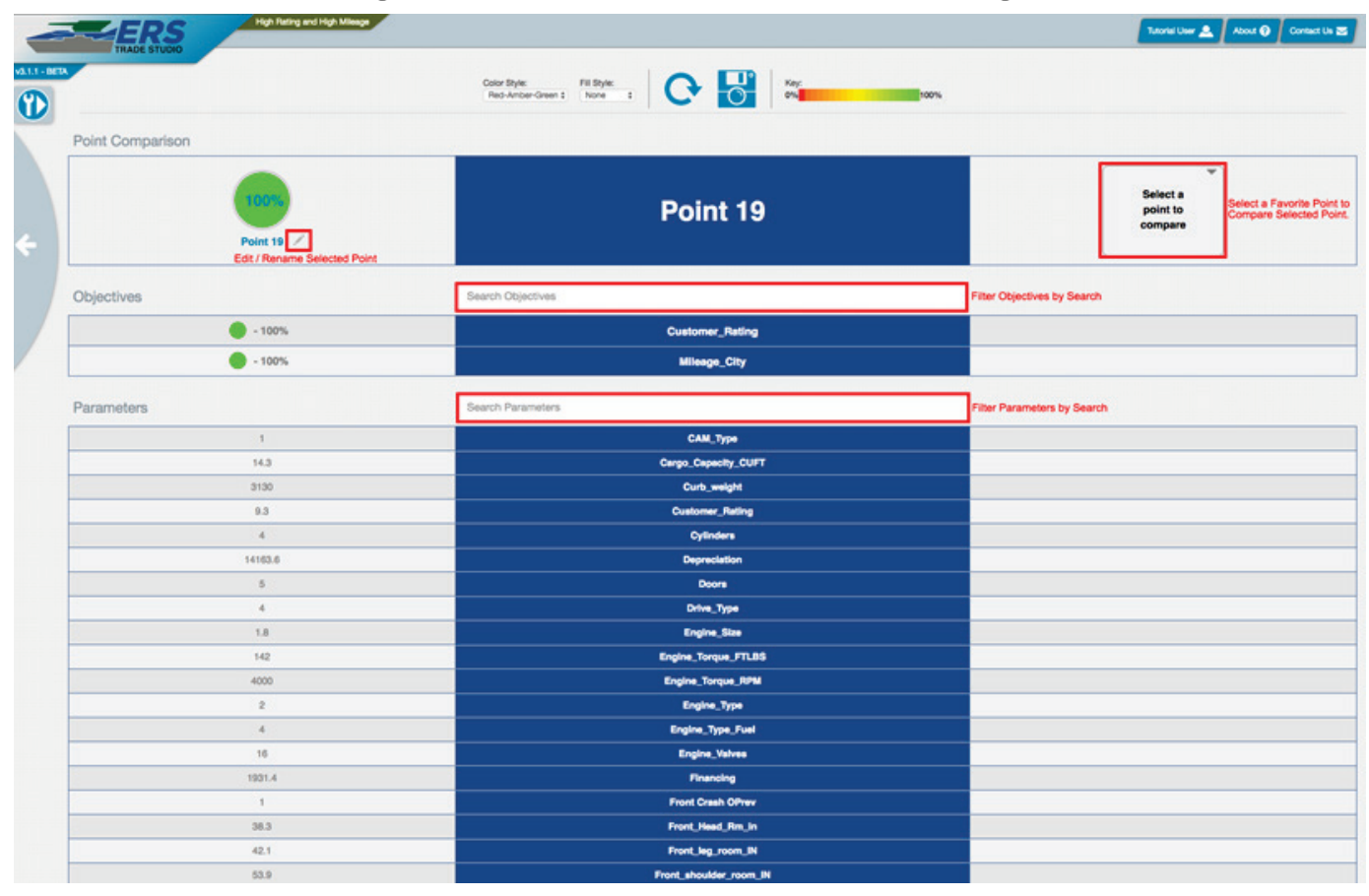

The following is an outline of the features labeled in Figure 60:

- Edit/Rename Selected Point - Allows the user to rename the point.

- Search Objectives - Allows the user to filter objectives by the search query.

- Search Parameters - Allows the user to filter parameters by the search query.

- Select Comparison Point - This allows the user to select a favorite point and compare it to the currently selected point. This functionality cannot be utilized if there are no favorite points. To make a data point a favorite point, the user must click on the star symbol within the point (Figure 61). Once a point has been selected, it will show up on the page. 
Figure 61. Select Tool Points Selected for Comparison.

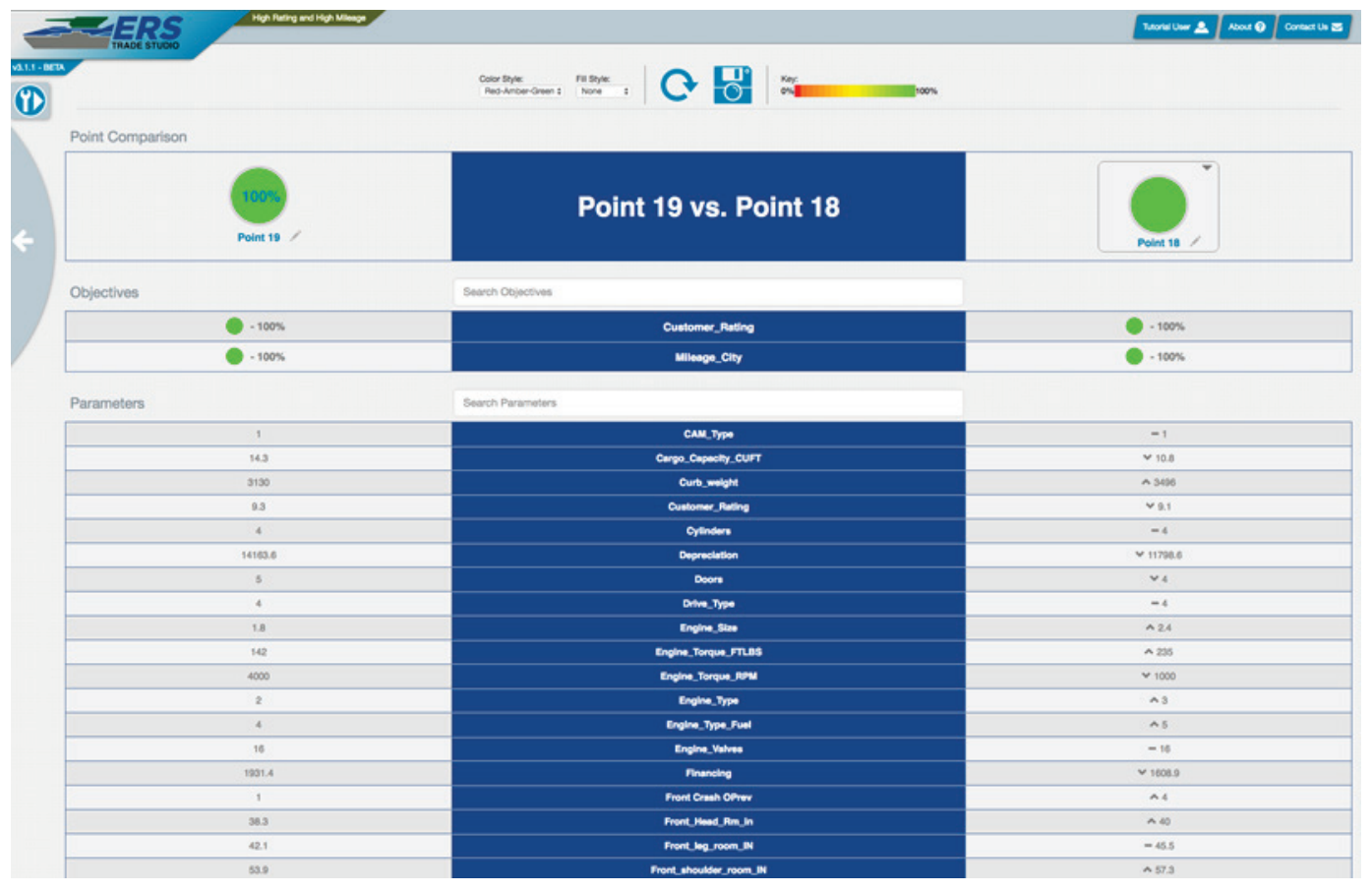

The left column represents the point that has been selected from the previous page; whereas the right column represents the favorite point selected for comparison. The middle blue column serves as the labels for the given values. Within each column, the parameters and objective values will be visible for comparison. 


\section{Explore Tool}

The Explore tool creates a graph that allows users to explore their tradespace by selecting various plots (Figure 62). These plots are rendered in a browser using the Plotly library (https://plot.ly/), therefore, they are limited by the size of the data.

The Explore tool can be reached by clicking Explore the tool orbit or by selecting the Plotly Charts selection in the Combine tool.

Figure 62. Explore Tool home page.

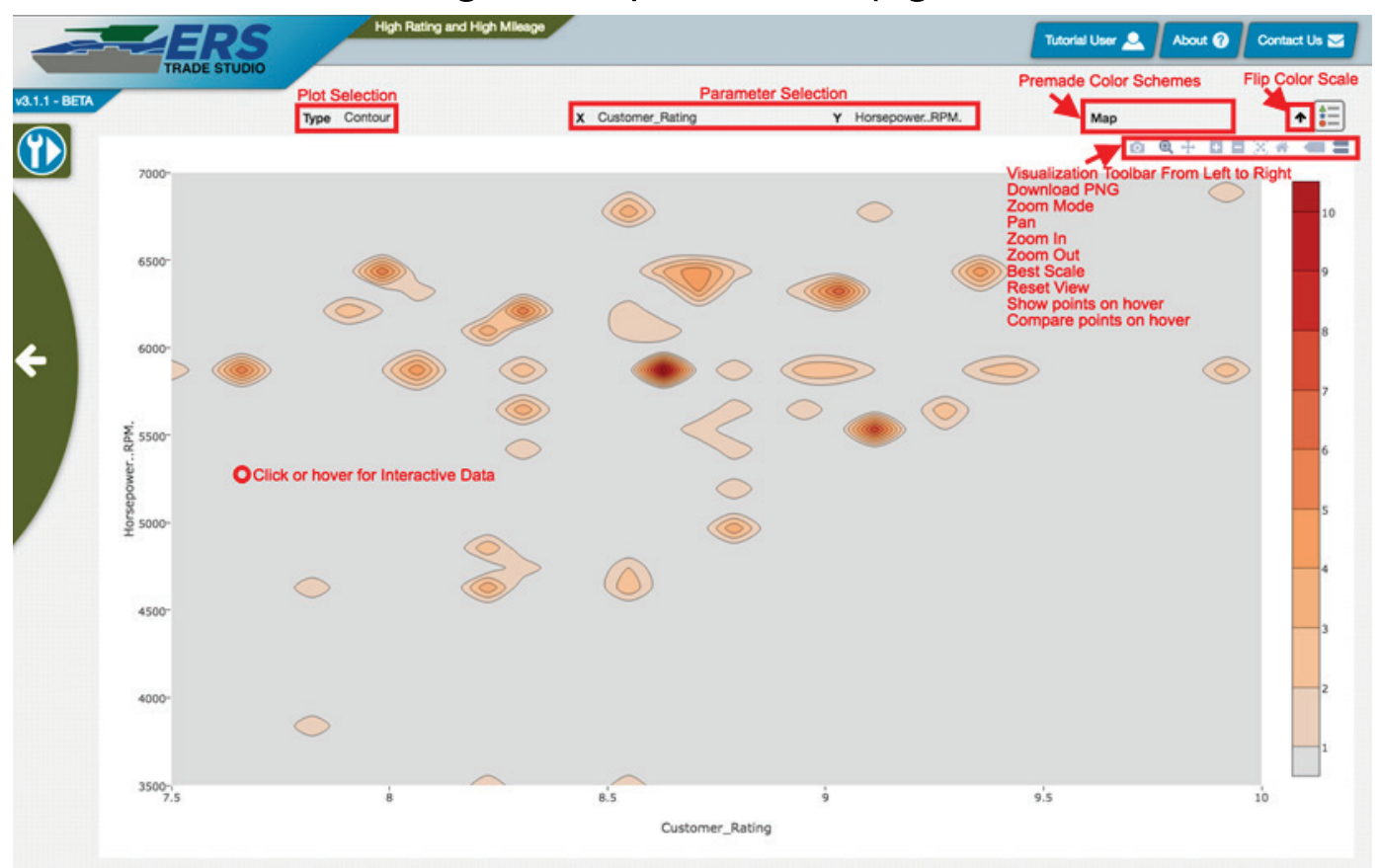

When a user first arrives to the Explore tool page, a graph may or may not have data showing, depending on the default parameters. However, the following tools will allow the examination of data:

- Plot Selection - The Type drop down changes the chart. There are seven different types which will be discussed later.

- Parameter Selection - The X and Y drop down change the axes of the visible chart to any selected parameter in the tradespace.

- Change Color Schemes - The Map drop down changes the color for the visible chart. Some charts do not have color maps, and specifically the Trend visualization will be replaced with Axes Sampling, this will be covered later. 
- Flip Color Scale - The color scale on the right of the chart will be flipped.

- Visualization Controls - This toolbar provides various ways to interact with the graph and has the following abilities, in order of left to right (download plot as a .png, zoom, pan, zoom-in, zoom-out, autoscale, reset axes, show closest data on-hover, and compare data on-hover).

The user has an option of the following seven different visualizations:

- Contour - A view of point density using curved edges around similarly dense areas (Figure 63).

Figure 63. Explore Tool contour view.

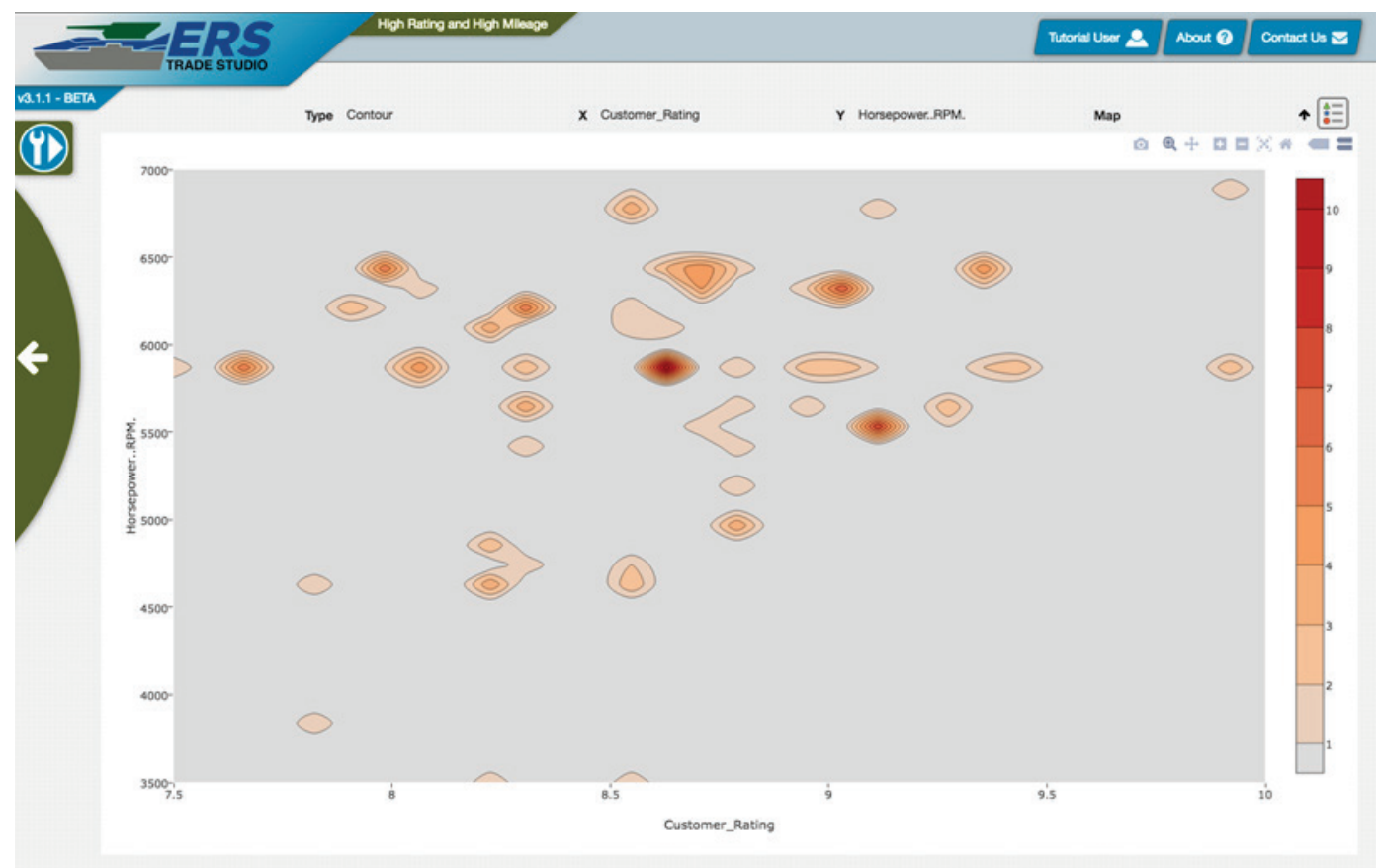

- Heatmap - A view of point density using a uniform grid and counting the number of points in each tile (Figure 64). 
Figure 64. Explore Tool Heatmap view.

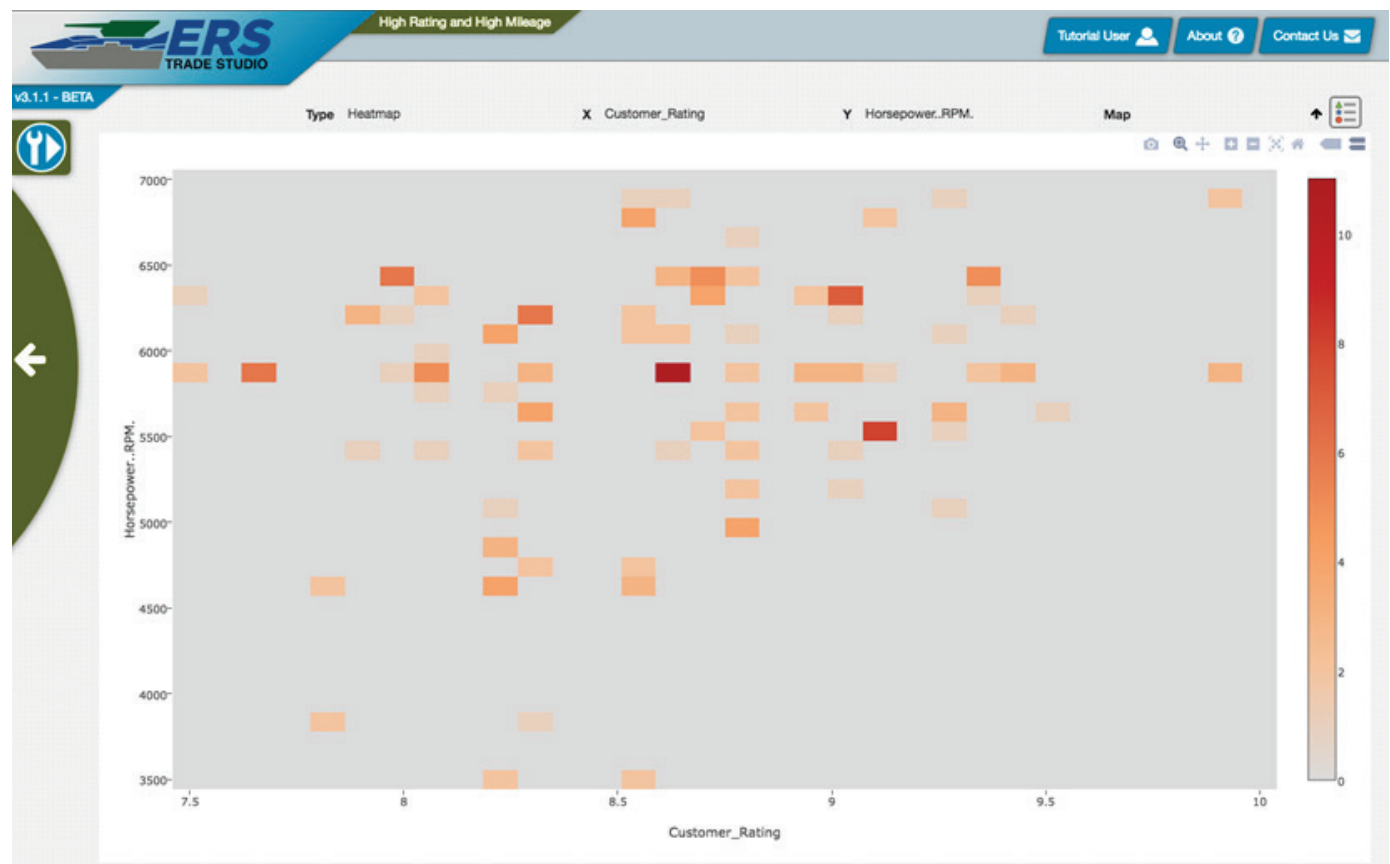

- Scatter - A view of point density using a uniform grid similar to heatmap, except replacing tiles with individual points (Figure 65).

Figure 65. Explore Tool Scatter view.

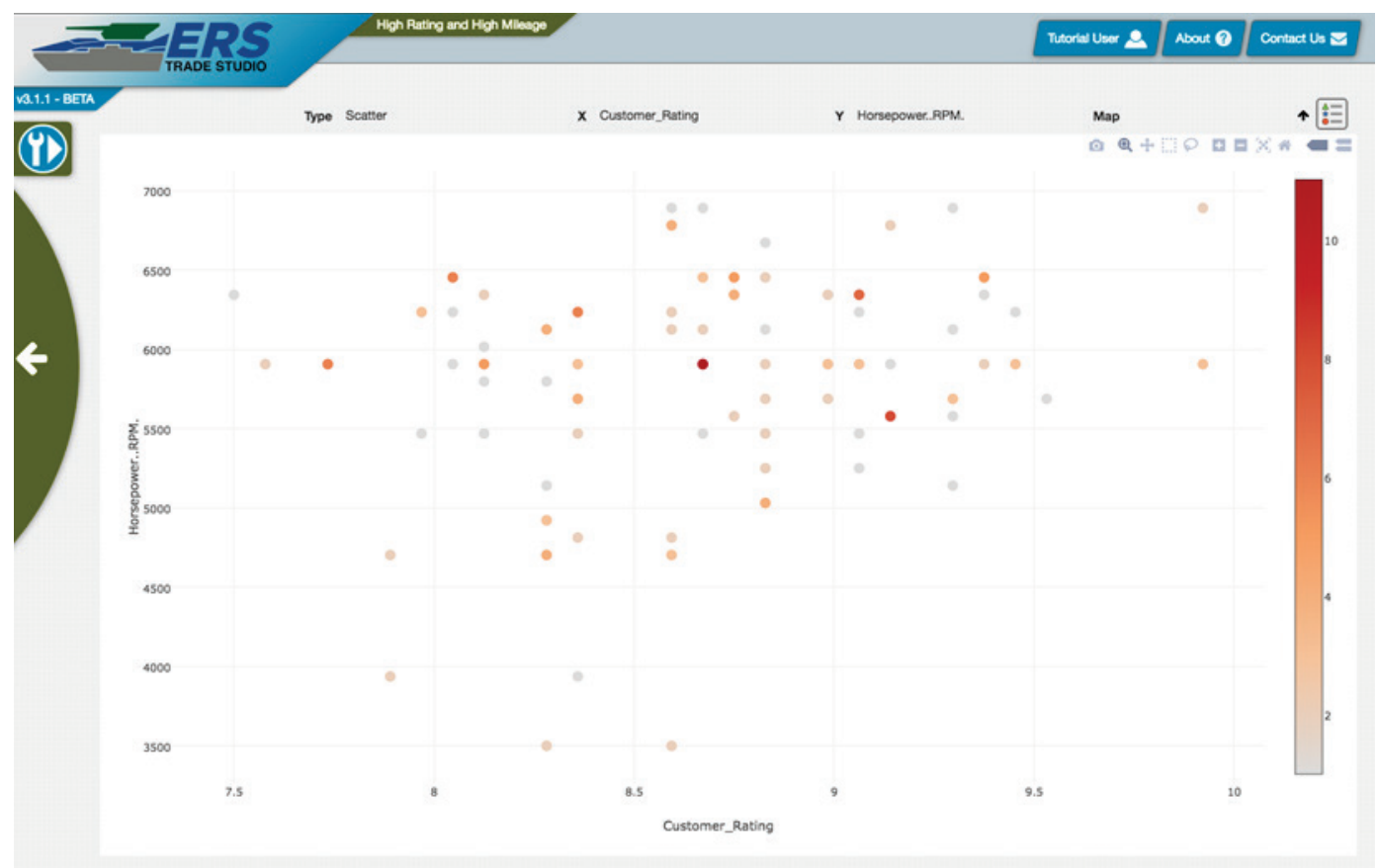

- ScatterXY - A basic scatterplot showing all of the points in an X-Y graph (Figure 66). 
Figure 66. Explore Tool Scatter view

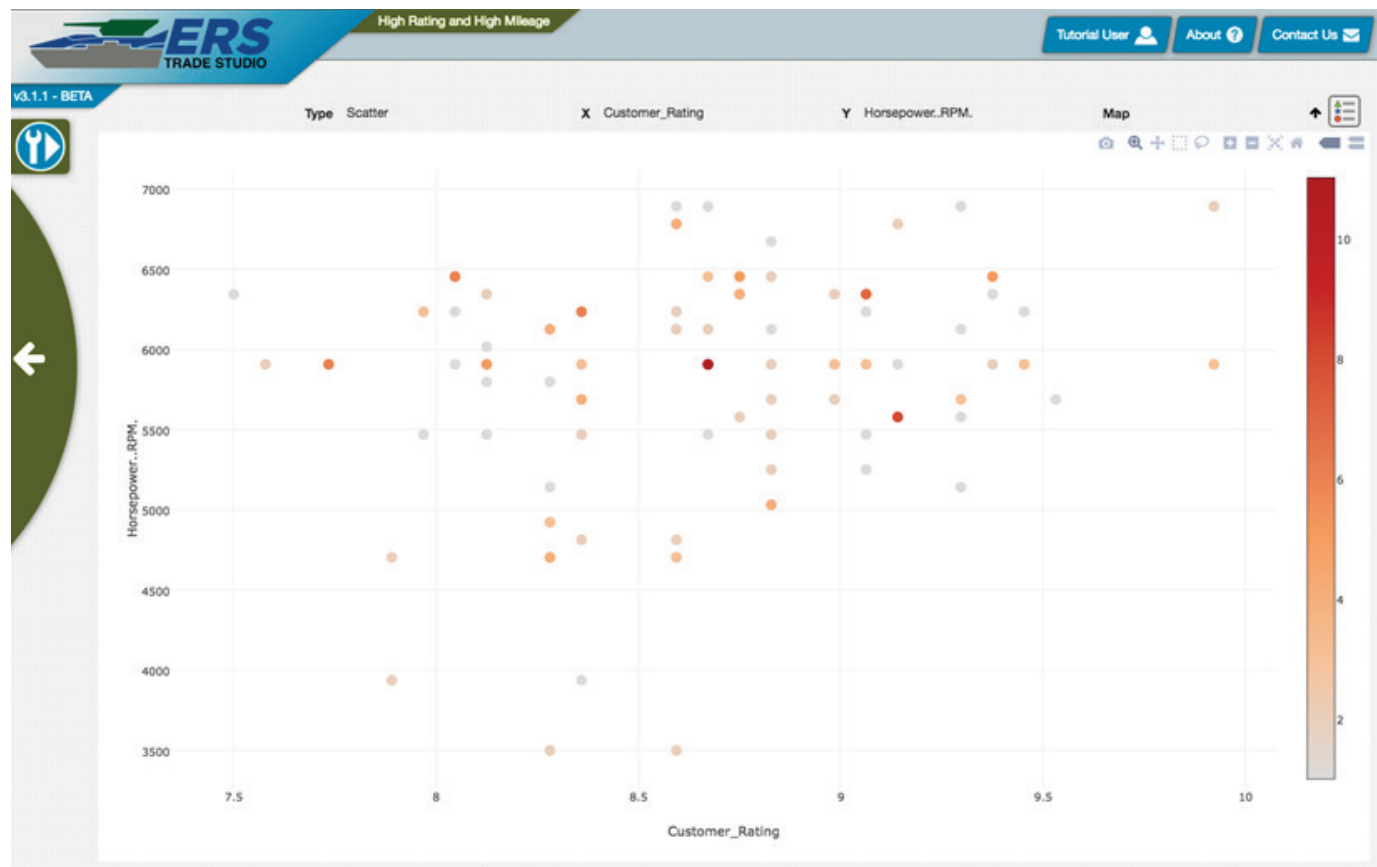

- ScatterXY - A basic scatterplot showing all of the points in an X-Y graph (Figure 67).

Figure 67. Explore Tool ScatterXY view.

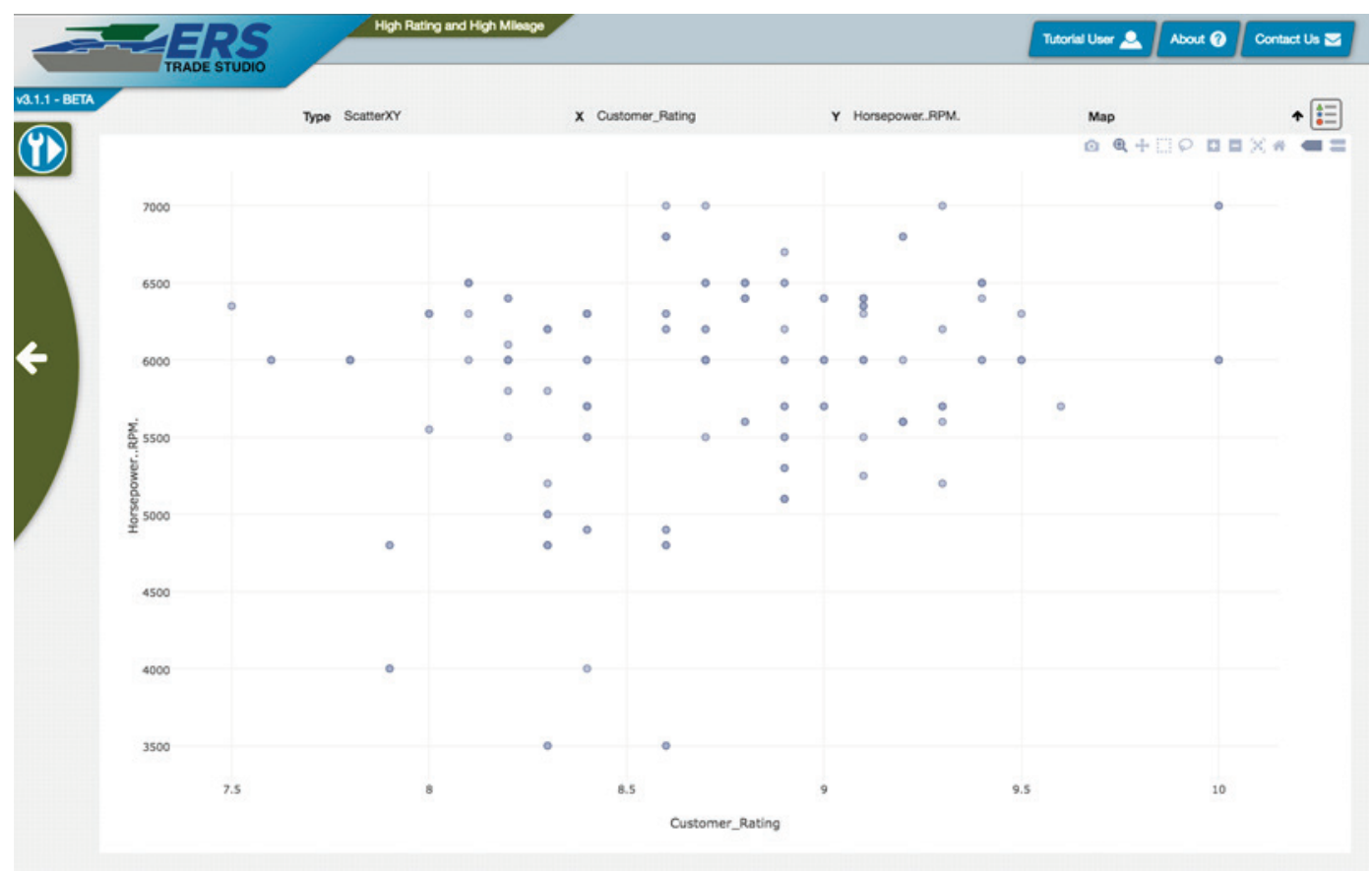

- ScatterXYContour - The ScatterXY plot overlaid on the Contour plot (Figure 68). 
Figure 68. Explore Tool ScatterXYContour view.

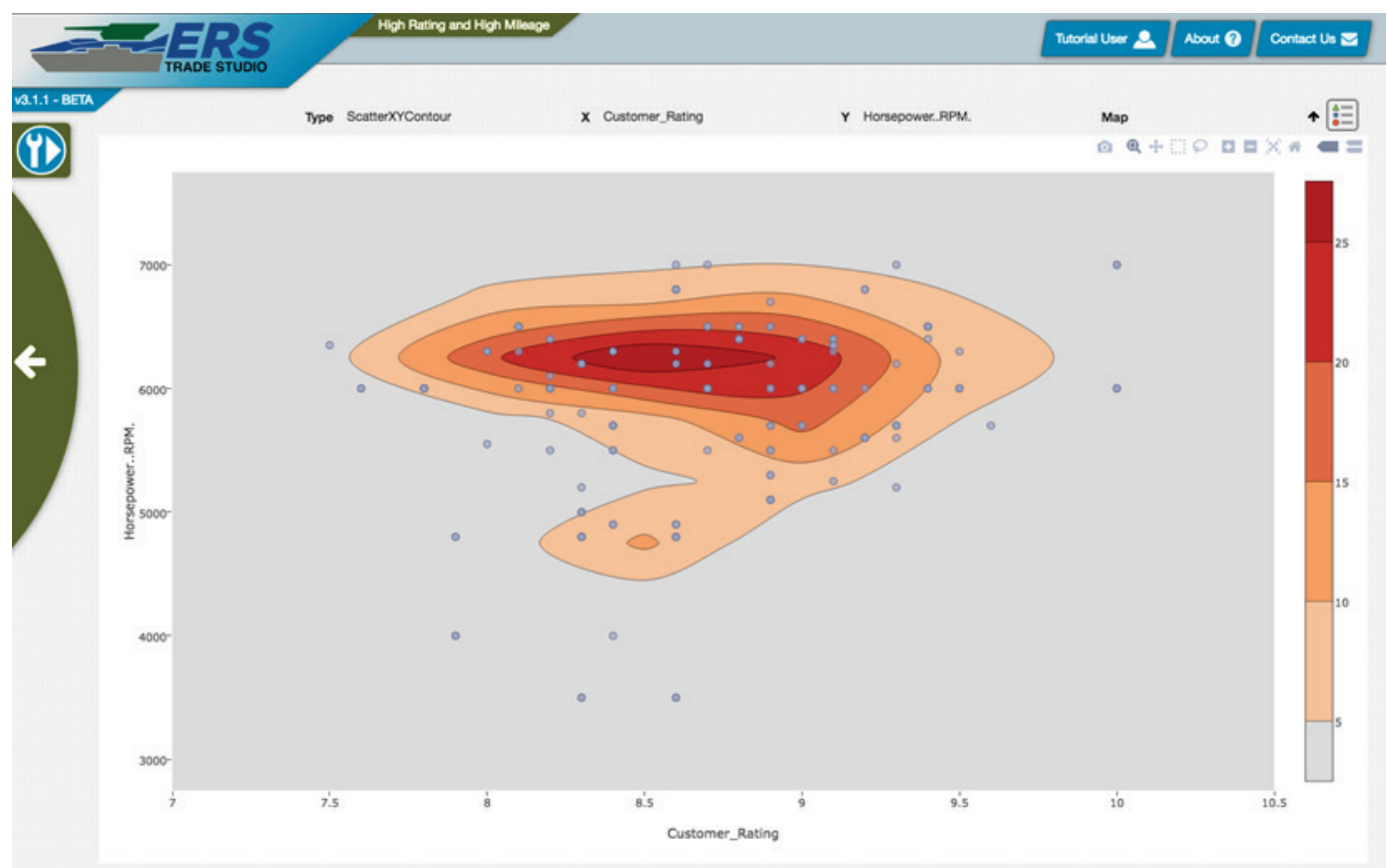

- Surface $3 D-\mathrm{A} 3 \mathrm{D}$ visualization showing density. The height of the plot reflects how many points are in that area. This uses WebGL to render the plot, utilizing the browser's rendering capability. The performance of this tool is dependent on the user's computer capability (Figure 69).

Figure 69. Explore Tool Surface3D view.

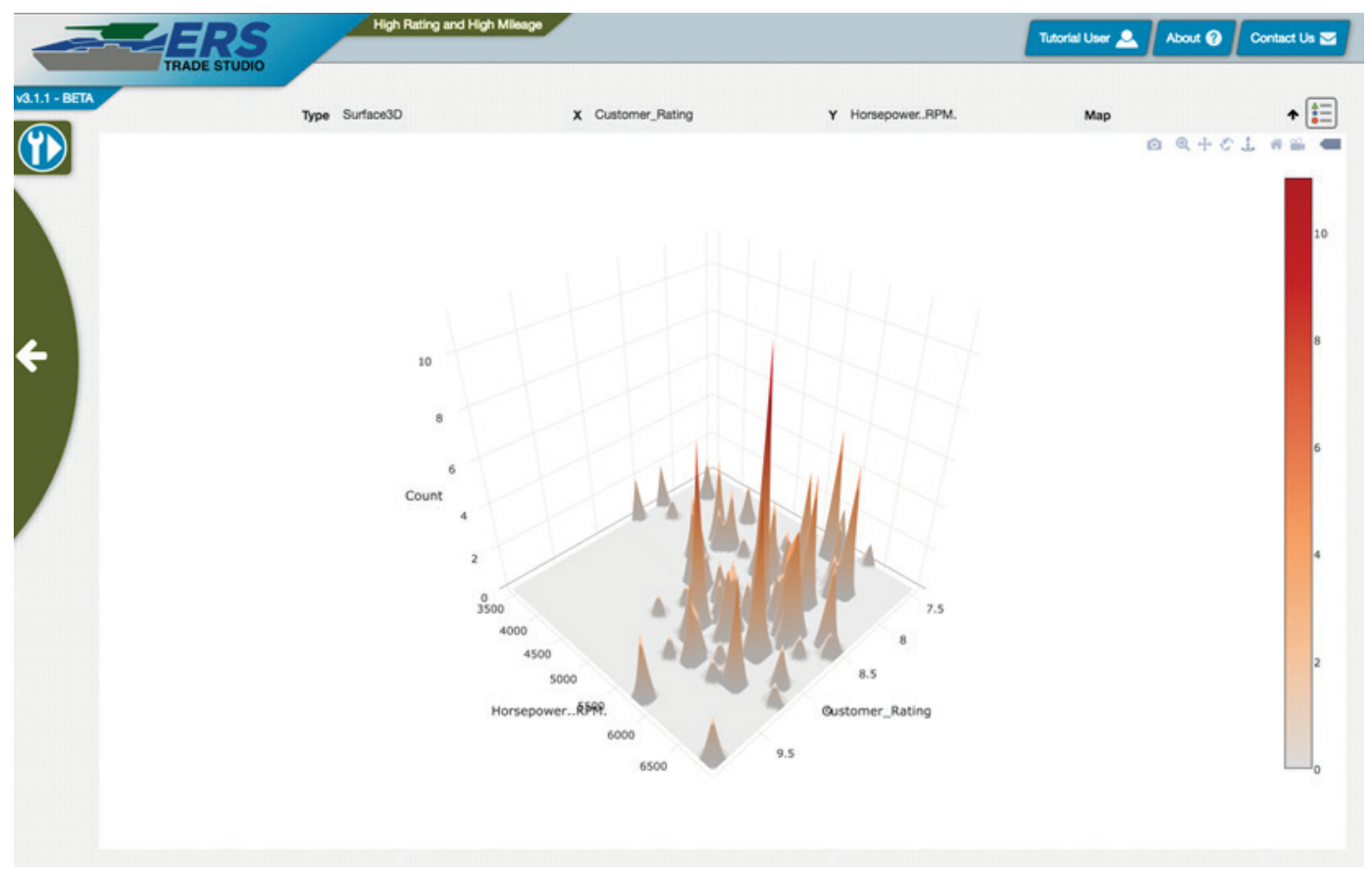


- Trend - A visualization of various statistical measures. This visualization's capabilities will be covered more in depth further in this chapter (Figure 70).

Figure 70. Explore Tool Trend Visualization Statistics.

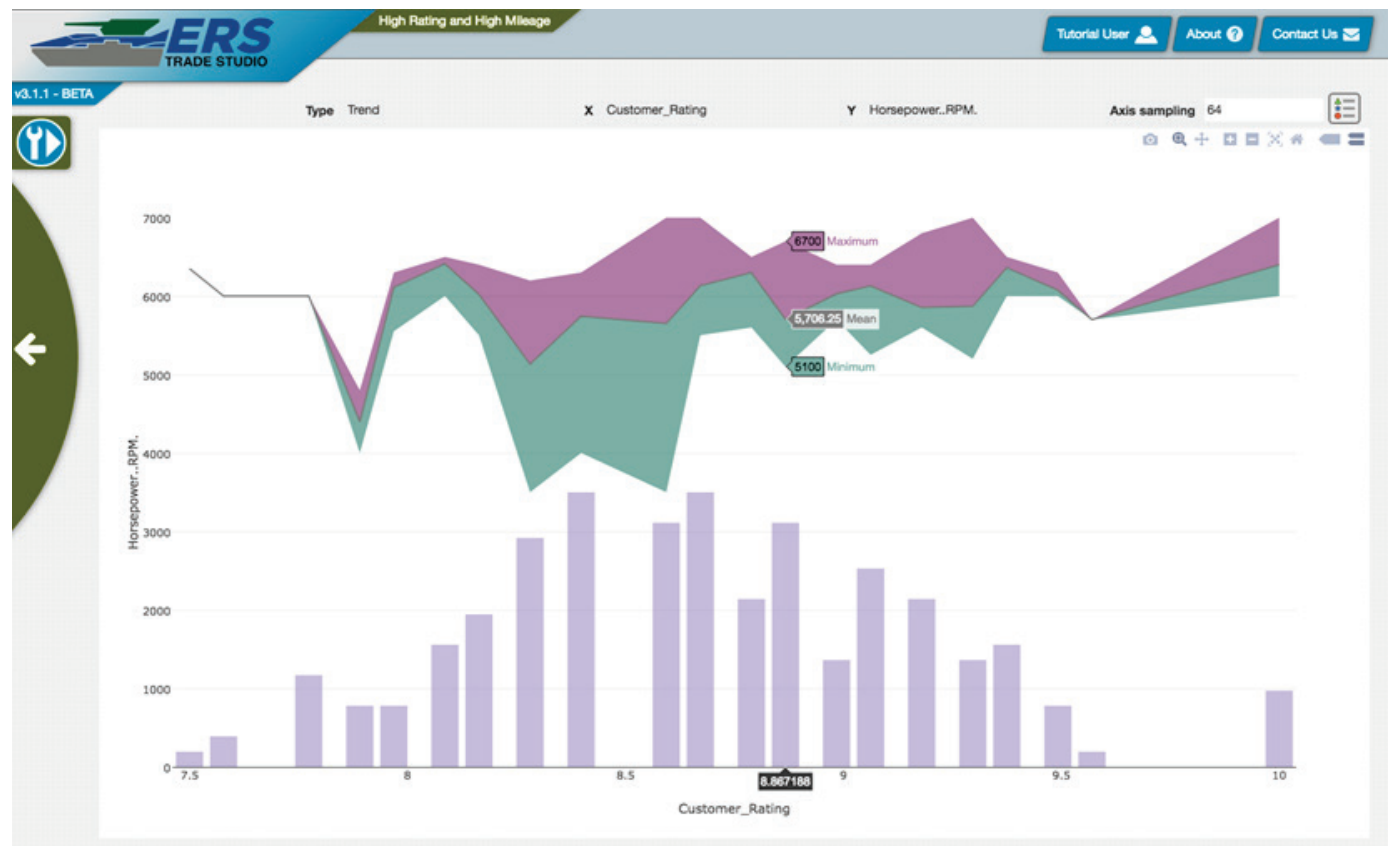

For the Trend tool, a user can zoom in on a specific area in the chart by clicking, holding down the mouse button, and dragging until the area that the user wants to analyze is in a box. To zoom back out, the user can double click the graph or click the zoom out button in the top right corner.

The user can adjust the size of the chart by scrolling up or down while hovering over the chart.

The user can move the chart around by holding down shift, clicking an area, and holding down the left mouse button while dragging around the cursor. This will move the chart around. To return the chart to its original view, the user can double click anywhere on the chart.

The $\mathrm{X}$ axis of the graph represents the number of $\mathrm{X}$ variables that occur, and the $\mathrm{Y}$ axis represents the number of $\mathrm{Y}$ variables that occur.

Since that data by itself is not valuable, there is also a shaded area. This is seen in Figure 69, where the purple and green shaded regions provide a user with additional information. When a user left clicks a point on the graph, the Maximum, Minimum, and Mean value associated with the $\mathrm{X}$ and $\mathrm{Y}$ axis intersection that was clicked will be shown in this area. 


\section{Customize Tool}

TradeAnalyzer has numerous ways for visualizing and reducing your Tradespace data. However, there will still be times when the available tools do not cover every use case. In these instances, the R-Analytics tool can be used. R-Analytics gives you the ability to add custom functionality to TradeAnalyzer for your specific tradespace.

The $\mathrm{R}$ in "R-Analytics" refers to the $\mathrm{R}$ programming language. $\mathrm{R}$ is a free software environment for statistical computing and graphics. To optimize use of the R-Analytics tool, first install RStudio on your local machine.

Begin by opening RStudio and installing the ERS-R-Tools library (visit https://public.git.erdc.dren.mil/ers/ERS-RStudio-Tools for additional up to date instructions). This resource will help write a valid R-Script that can be uploaded to TradeAnalyzer. This guide does not detail writing R scripts, but will focus on the usage of the web application. The ERS-RStudio-Tools repository also has a collection of example scripts that can be referenced and even uploaded for use. It is recommended to download and use one of those examples if the process of writing a script is not needed.

Once the user has either written or acquired the script, open the RAnalytics tool in TradeAnalyzer. When the tool is first opened, there will be three icons displayed (Figure 70). 
Figure 71. R-Analytics Tool.

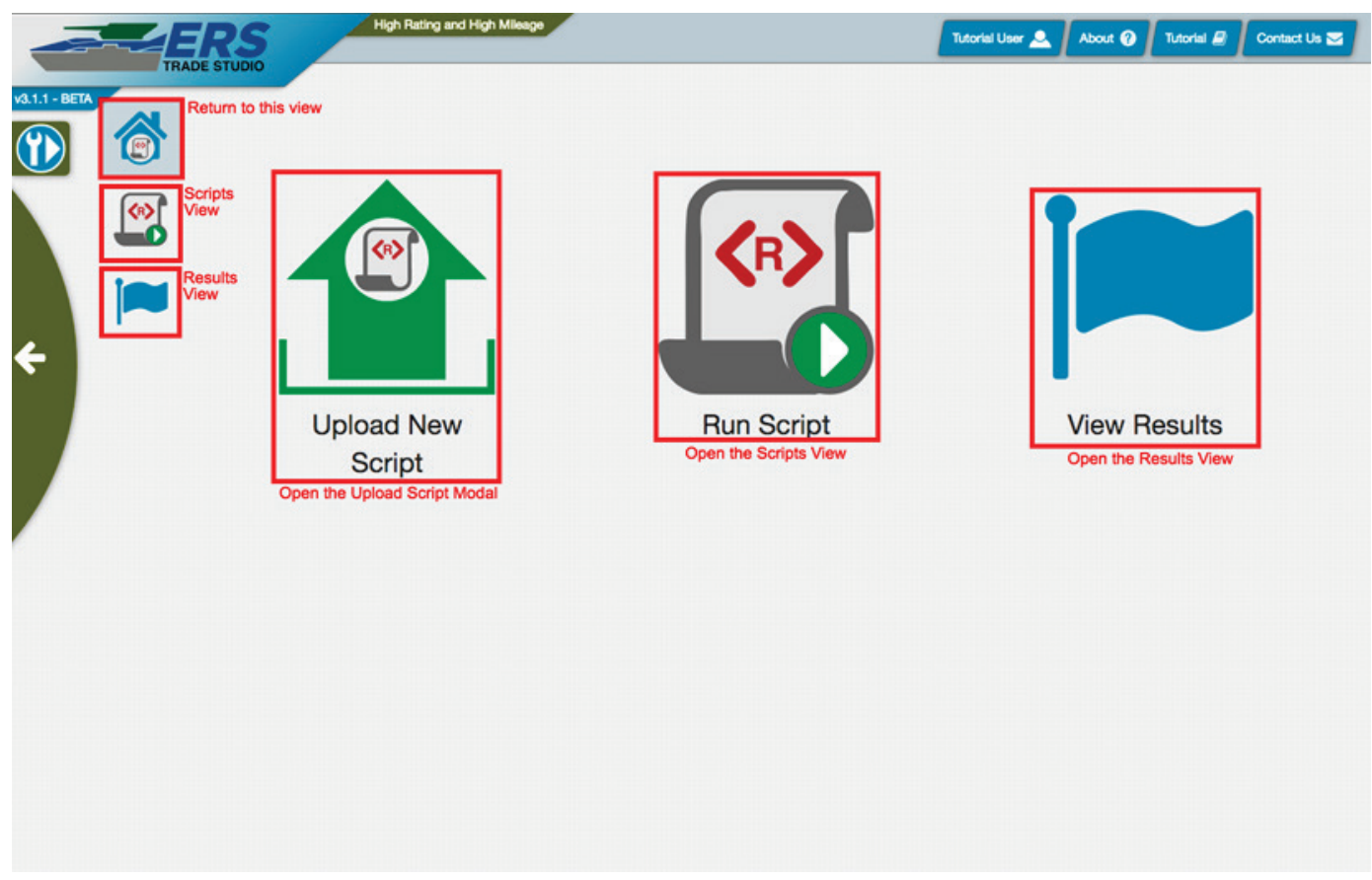

- Upload New Script - will open a modal for the user to upload a new script.

- Run Script - will present the user with a list of uploaded scripts to select and run.

- View Results - will take the user to a list of results from scripts that have already ran.

To upload a new script, click the icon Upload New Script.

Clicking the Upload New Script icon will open the Script Upload Modal shown in Figure 71. The R-Analytics Choose File button will allow the user to select a file with an extension of .r. Select the script you wish to upload and give it a short but meaningful name and an optional description. Click Next and this will upload your script and begin the validation process. This can take some time, however, the user is free to leave the application and come back later. Once the script is finished, it will appear in the Select Script list under the Run Script view. Sometimes scripts can run into errors when validating. The server will send the user a notification of script upload failure and attempt to provide a meaningful error message to help the user understand how to correct the script and try again. 
Figure 72. R-Analytics script upload.

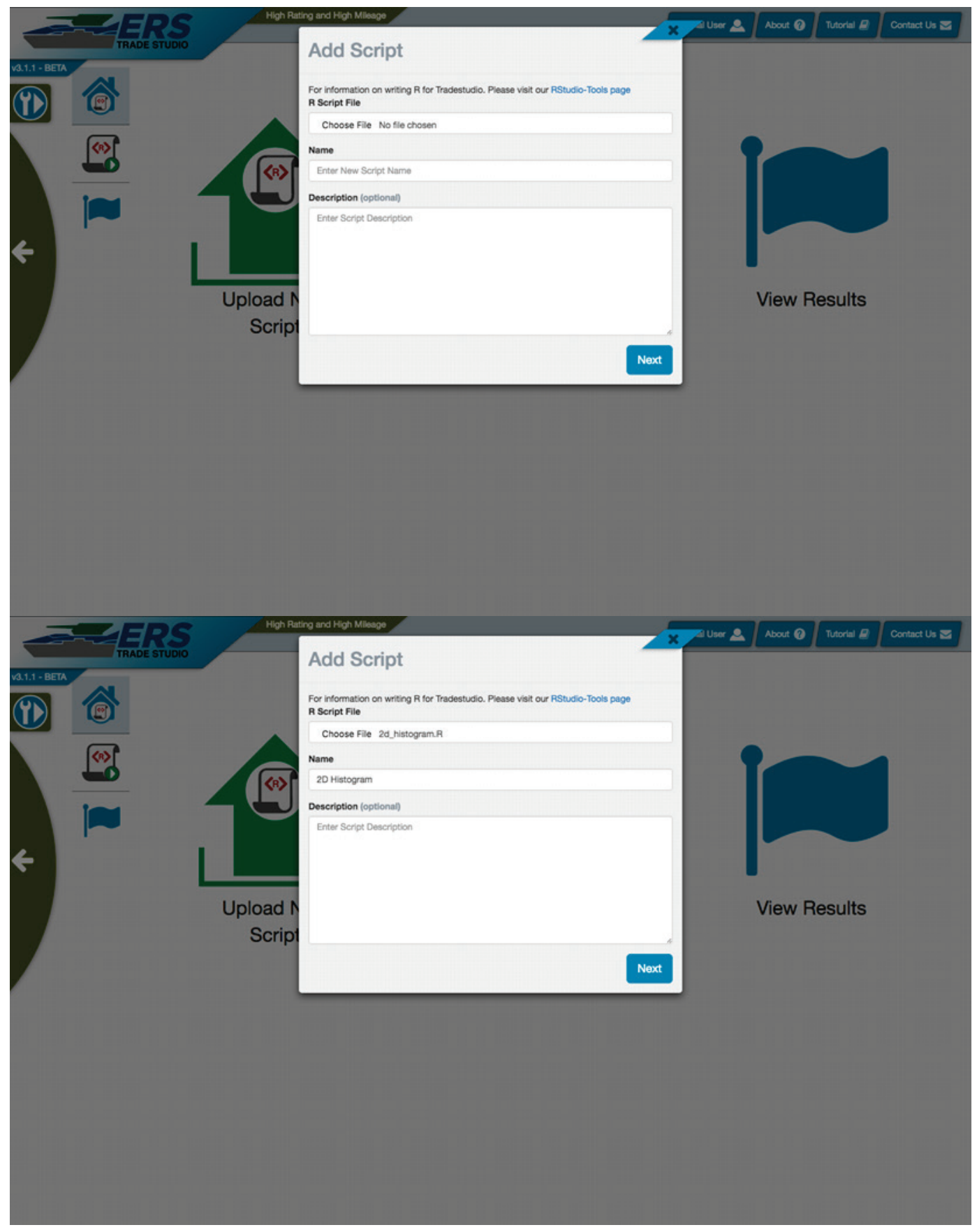

Now that the script has been uploaded, it can be selected or deleted. Clicking the trashcan icon in the top right of the script card will remove the script from the project. Clicking on any other part of the card will open the scripts parameter form and allow the user to select and provide parameters (Figure 73). 
Figure 73. R-Analytics select script.

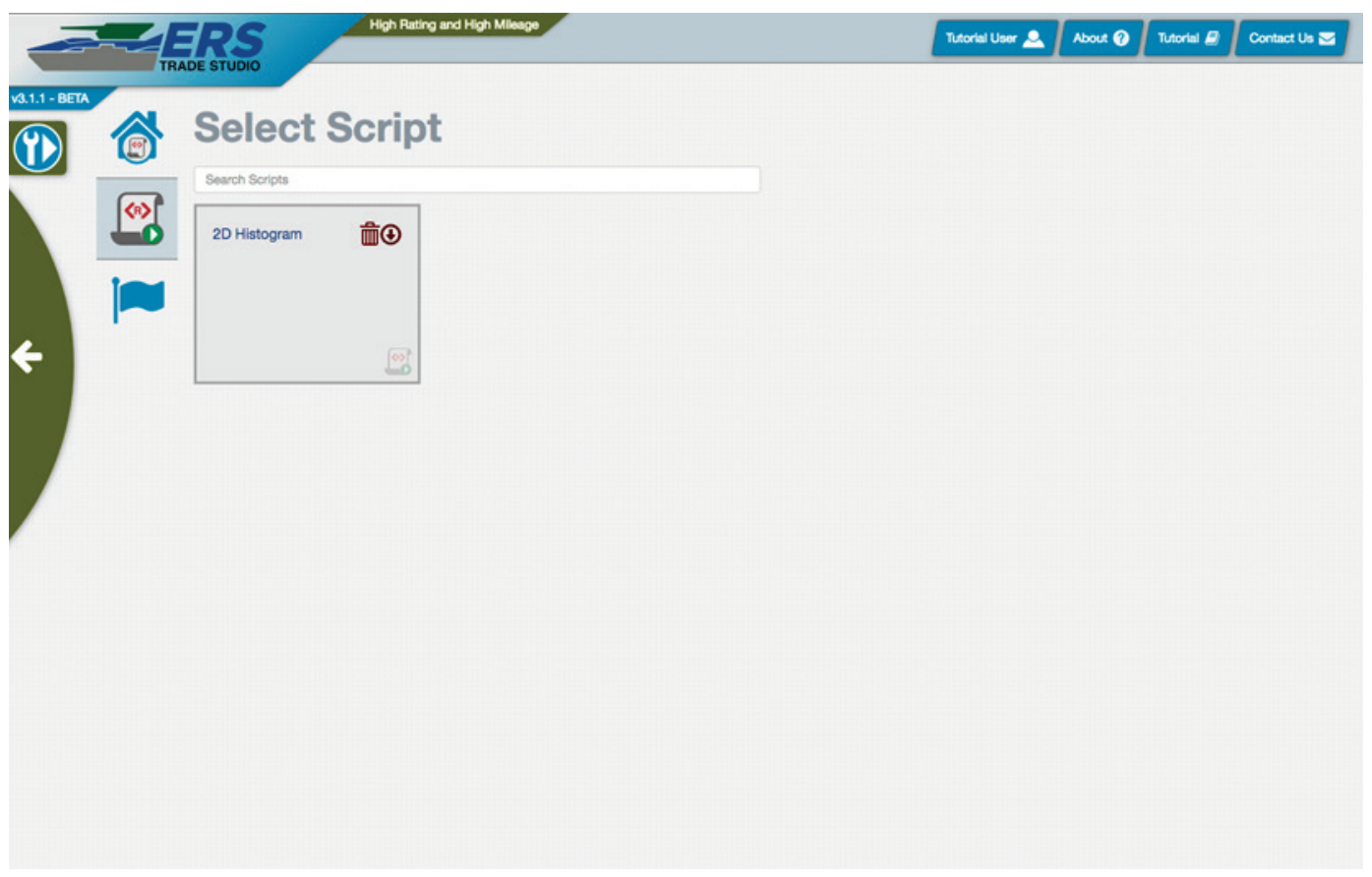

Running a script will always require providing a result name. If not entered, the result name will be the name of the script along with a timestamp of when the script was started. The other parameters are dynamically generated based on what inputs the script required. The user may be asked to enter various types of inputs that are relevant to the analysis or visualization that is being executed.

Fill in all of the required fields for your particular script and then click the Run Script button (Figure 73). 
Figure 74. R-Analytics run script.

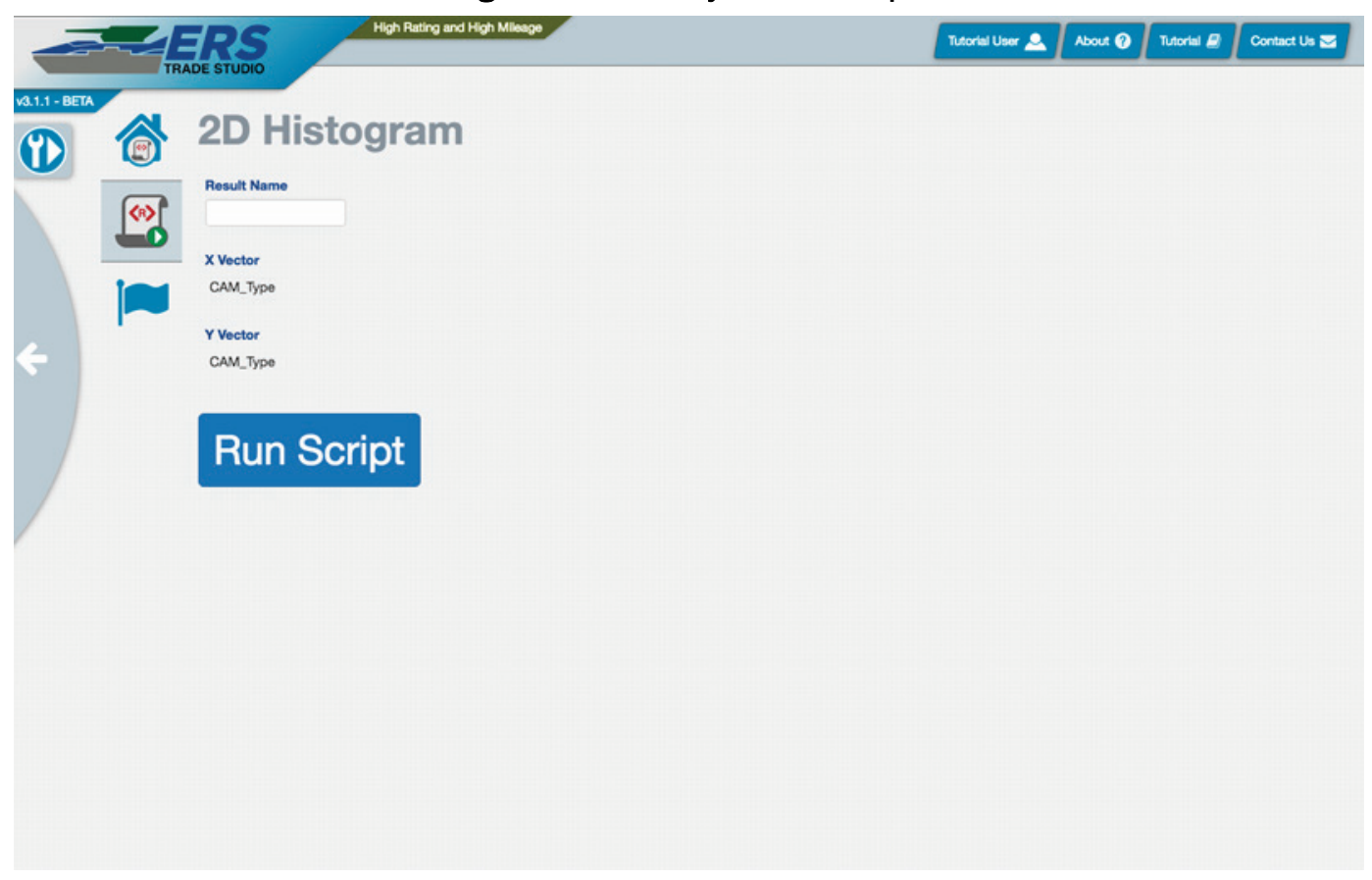

Once the user clicks Run Script, the script will be executed with the desired parameters. Depending on the nature of the script and the size of the data, this could take anywhere from a few seconds to several hours. The Results view will show the results that have been run, the results that are currently running, and the results that failed.

Successfully completed results will appear as green, clicking them will show the user the images that were produced by the script or information about a sub tradespace that was generated and exported by the script (Figures 75 and 76).

Pending results will appear as yellow and will not be clickable until they have completed. When the result is complete, the card will automatically switch from yellow to green without having to refresh the page.

Failed results will appear as red and clicking them will show information about what happened during execution. 
Figure 75. R-Analytics view results.

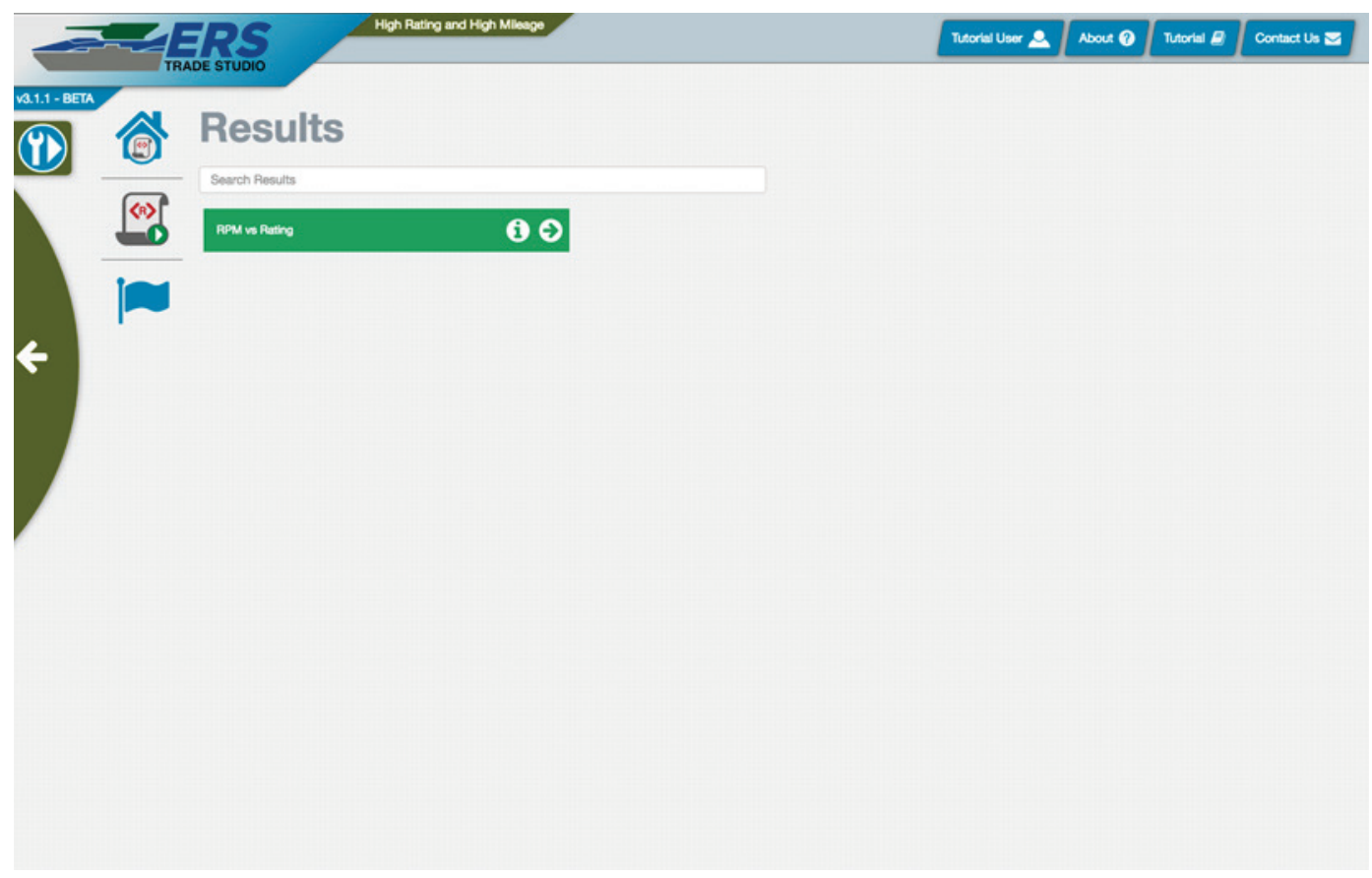

Figure 76. R-Analytics view results histogram result

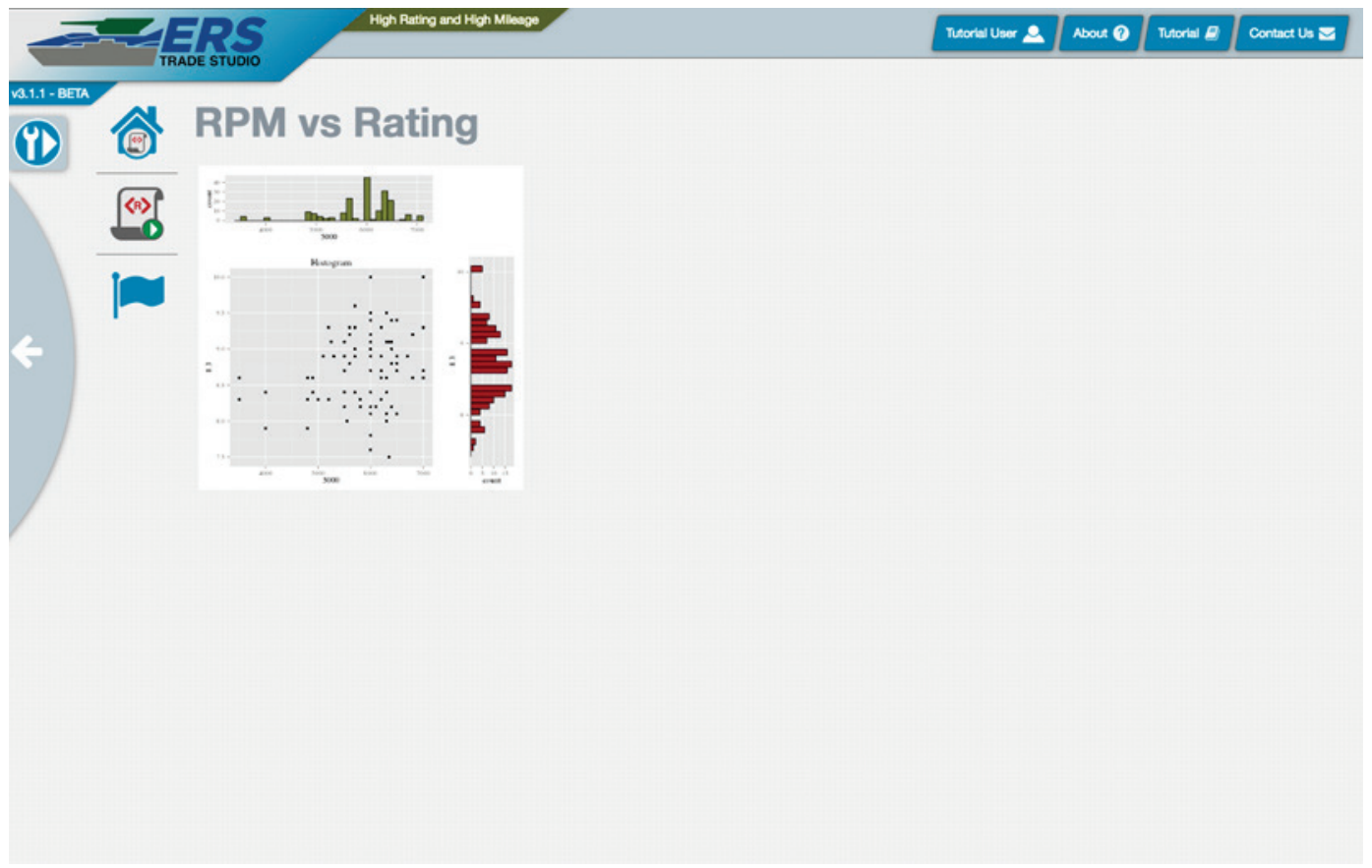




\section{Frequently Asked Questions}

Q: I visited the site and suddenly everything appears out of place, what do I do?

A: This is usually an issue with the browser cache. In this situation, the best solution is to clear the browser cache. To find out how to clear the browser cache, please see the following guide:

http://www.refreshyourcache.com/en/home/

After clearing the browser cache, the browser can be closed and reopened, and the issue should disappear.

Q: I uploaded a .csv and after a few minutes it still says pending. Is this normal behavior?

A: The amount of time that a .csv takes to finish processing can vary, and depends on the size of the .csv, the number of columns in the .csv, and the network speed. Large csv's, or .csv's uploaded on networks with limited network capability, can take hours to complete. This is normal behavior.

Q: The 3D scatterplot seems very slow, but I only have a few hundred points. Why?

A: This is usually an issue with network connectivity, however, a decreased performance on large monitors has been noted. If the latter is the problem, the browser window can be reduced in size. 


\section{Where to Find Further Help}

To submit application feedback or ask questions about TradeAnalyzer, contact us through email at ers.suppor@erdc.dren.mil or by selecting Contact Us in the TradeAnalyzer application. 


\section{Conclusion}

This report serves as a user guide to understanding the various views and tools in TradeAnalyzer. The guide provides thorough explanations and example use cases of the many tools TradeAnalyzer provides. 


\section{Appendix A: Glossary of Terms}

Browser - Software used to access the World Wide Web (e.g., Google Chrome, Internet Explorer, and Firefox are examples of browsers).

Common Access Card (CAC) - The CAC is a smart card that serves as identification for DoD or military personnel. It can be used to access buildings and computers in eligible spaces.

Chart - A visual representation of data, in which the data is represented in a form that is easy to understand and share. Various types of charts exist, such as bar charts, line charts, pie charts, etc. See also: graph-

Column - A vertically divided set of data that corresponds with one or more rows. Sometimes has a header to describe what type of data will fall within the data set. See also: parameter.

Field - The intersection of a row and column. (e.g., if a file has three rows and three columns, it has nine fields). In some software architectures, may also be used in place of the term column.

Graph - A visual representation of data, in which the data is represented in a form that is easy to understand and share. Various types of graphs exist, such as bar graphs, line graphs, pie graphs, etc. See also: chart.

Image - A physical representation or likeness of an object or idea that be seen.

Mutual Information -reduction in uncertainty (entropy) due to another parameter. It is a measure of the dependence between two parameters.

Package - A subset of data related to a tradespace. See also: Trade subset

Parameter - A vertically divided set of data that corresponds with one or more rows. Sometimes has a header to describe what type of data will fall within the data set. See also: column

Project - A project is one or more tradespaces related to a particular problem. 
Python - A high level interpreted programming language for generalpurpose programming. Python is widely used in scientific computing and has a core philosophy focusing around readability, simplicity, and explicitness.

$\mathrm{R}$ - A programming language used in graphics and statistical computing. Data scientists, data miners, and statisticians use $\mathrm{R}$ for developing data analysis and data mining software.

Row - A row is a horizontal set of data that has information from each parameter or column.

Sankey - A type of flow diagram that creates a visualization of the major flows between data in a set of information.

State - Information related to previous user actions or events that is remembered by software (e.g., a web application may return a user to the last page they were viewing with state).

Tradespace - A set of data in which the parameters have strong interdependencies. A tradespace is traversed to discover optimal solutions to problems with regard to particular goals and objectives while observing the costs or trade-offs of particular parameters and how they relate to one another (e.g., in TradeAnalyzer, the tradespace would be the data contained within the .csv, where each column would be a parameter).

Trade subset - A subset of data within a tradespace. See also: package. 


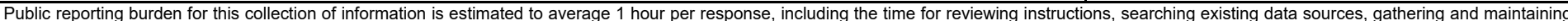

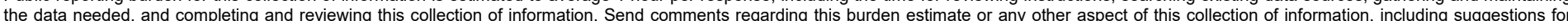

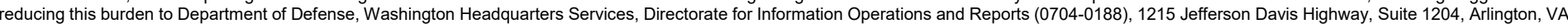

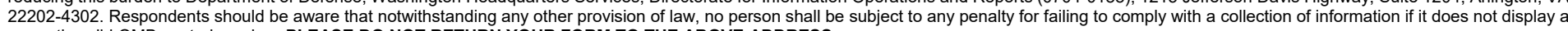
currently valid OMB control number. PLEASE DO NOT RETURN YOUR FORM TO THE ABOVE ADDRESS.

\begin{tabular}{|l|l|l|} 
1. REPORT DATE (DD-MM-YYYY) & 2. REPORT TYPE & 3. DATES COVERED (FrOm - To)
\end{tabular} July 2019

\section{TITLE AND SUBTITLE} SR

TradeAnalyzer v4.o User Guide

5a. CONTRACT NUMBER

5b. GRANT NUMBER

5c. PROGRAM ELEMENT NUMBER

\section{AUTHOR(S)}

5d. PROJECT NUMBER

Eric R. Mixon, Joshua Q. Church, Kenneth D. Niles, and Timothy W. Garton

5e. TASK NUMBER

5f. WORK UNIT NUMBER

92L5D8

\section{PERFORMING ORGANIZATION NAME(S) AND ADDRESS(ES)}

8. PERFORMING ORGANIZATION REPORT NUMBER

Information Technology Laboratory

U.S. Army Engineer Research and Development Center

ERDC/ITL SR-19-1

3909 Halls Ferry Road

Vicksburg, MS 39180-6199

\section{SPONSORING / MONITORING AGENCY NAME(S) AND ADDRESS(ES)}

Headquarters, U.S. Army Corps of Engineers

Washington, DC 20314-1000

10. SPONSOR/MONITOR'S ACRONYM(S)

ERDC

11. SPONSOR/MONITOR'S REPORT NUMBER(S)

\section{DISTRIBUTION / AVAILABILITY STATEMENT}

Approved for public release; distribution is unlimited.

\section{SUPPLEMENTARY NOTES}

\section{ABSTRACT}

This document describes the usage of TradeAnalyzer, a subcomponent of the overarching Engineering Resilient Systems (ERS) tool suite known as TradeStudio. TradeStudio is a generalized and reusable workflow engine used for accelerating common tradespace assembly tasks. TradeAnalyzer supports the ERS effort by developing more comprehensive and complete tradespaces, facilitating Department of Defense (DoD) conceptual design pipelines to the High Performance Computer (HPC), and by solving cross cutting issues in the design process.

TradeAnalyzer serves as an enterprise level web application designed to assist the user in visualizing and analyzing a tradespace. The analytics and visualizations provided act as a suite for decision support in down selecting to the best possible subset for the mission. The products provided by TradeAnalyzer include Large Data Analytics and Visualization (LDV) tools which use high performance, serverside rendering.

\begin{tabular}{|ll}
\hline 15. SUBJECT TERMS & $\begin{array}{l}\text { Systems engineering } \\
\text { System analysis }\end{array}$ \\
\end{tabular}

System analysis--Data processing Computer programs

\section{SECURITY CLASSIFICATION OF:}

\section{a. REPORT}

Unclassified

b. ABSTRACT
Unclassified

\section{c. THIS PAGE}

Unclassified

\section{NUMBER} OF PAGES 17. LIMITATION
OF ABSTRACT 19a. NAME OF RESPONSIBLE PERSON

19b. TELEPHONE NUMBER (include

90 area code) 